\title{
Holomorphic and de Rham torsion
}

\author{
Jean-Michel Bismut
}

\begin{abstract}
We prove the vanishing of the equivariant holomorphic torsion forms associated to the holomorphic de Rham complex. We also consider corresponding torsion forms associated to complexes in hypercohomology, for which we give a local formula. Rigidity of certain genera explains part of these formulas. The Lefschetz operators in Hodge theory play a prominent role in the proofs.
\end{abstract}

\section{Contents}

1 Clifford algebras and exterior algebras $\quad 1305$

1.1 A symplectic vector space . . . . . . . . . . . . . . . 1305

1.2 Clifford algebras and exterior algebras . . . . . . . . . . . . 1306

1.3 Complex structures and exterior algebras . . . . . . . . . . . . 1306

2 Kähler fibrations and Gauss-Manin connections 1309

2.1 Dolbeault and de Rham complexes . . . . . . . . . . . . . . . . . 1309

2.2 A Kähler fibration . . . . . . . . . . . . . . . . . . . . . . 1310

2.3 The de Rham operator of $M \ldots \ldots \ldots \ldots$. . . . . . . . . . . . . . . . . . . . . . . 1311

2.4 The holomorphic Hermitian connection on $T^{*} X \ldots \ldots$. . . . . . . . . 1312

2.5 The holomorphic Hermitian connection on $T X$. . . . . . . . . . . . 1313

2.6 The Dolbeault operators on $M \ldots \ldots \ldots \ldots$. . . . . . . 1314

2.7 The Gauss-Manin connection . . . . . . . . . . . . . . . . . . 1315

2.8 The exterior algebra $\Lambda^{*}\left(T^{*} X\right)$ as a twisting vector bundle . . . . . 1316

3 The Levi-Civita superconnections 1317

3.1 The holomorphic Levi-Civita superconnection . . . . . . . . . . . . . . . 1317

3.2 The de Rham Levi-Civita superconnection . . . . . . . . . . . . . . . . . 1318

3.3 A formula relating the Levi-Civita superconnections . . . . . . . . . . . 1319

4 The vanishing of the equivariant holomorphic analytic $\begin{array}{lr}\text { torsion forms } & 1321\end{array}$

4.1 A group action . . . . . . . . . . . . . . . . . . . . 1321

4.2 The holomorphic superconnection forms . . . . . . . . . . . . . 1321

4.3 The equivariant holomorphic analytic torsion forms . . . . . . . . . . . . 1324

4.4 The de Rham superconnection forms . . . . . . . . . . . . . . 1325

4.5 Comparing the holomorphic and de Rham superconnection forms . . . . 1325

4.6 An evaluation of $\left[\gamma_{t}\right] \ldots \ldots \ldots \ldots \ldots \ldots \ldots \ldots$

4.7 The vanishing of the analytic torsion forms . . . . . . . . . . . . . 1328

5 Double complexes and equivariant cohomology 1329

5.1 A holomorphic vector field . . . . . . . . . . . . . . . . . . . . . . . . . . . . . . . 1329

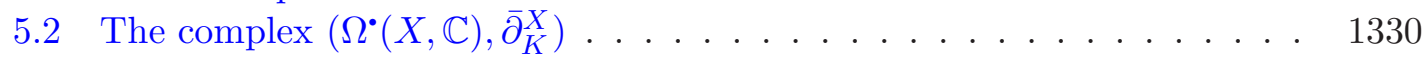

Received 14 November 2002, accepted in final form 26 June 2003.

2000 Mathematics Subject Classification 58J52 (primary), 58J20 (secondary).

Keywords: analytic torsion forms, index theory.

The author was supported by Institut Universitaire de France (IUF).

This journal is (c) Foundation Compositio Mathematica 2004. 
5.3 The adjoint operators . . . . . . . . . . . . . . . . . . . . . 1331

5.4 The $L_{2}$ metric on $\mathcal{H}_{K}^{\circ}(X, \mathbb{C})$ and the Bott localization formulas . . . . . 1332

5.5 The $L_{2}$ metric on the determinant of the cohomology . . . . . . . . . . . 1333

5.6 The $L_{2}$ metric on the equivariant determinant . . . . . . . . . . . . . . . 1334

5.7 The case where $K=f^{X} \ldots \ldots \ldots \ldots \ldots$. . . . . . . . . . . . . . . . . . . . . . . . . . . . 1334

5.8 A real flat line bundle . . . . . . . . . . . . . . . . . . . . . . . . . . . . . . . . 1336

5.9 The case where the action is Hamiltonian . . . . . . . . . . . . . 1337

5.10 An equivariant fibration . . . . . . . . . . . . . . . . 1338

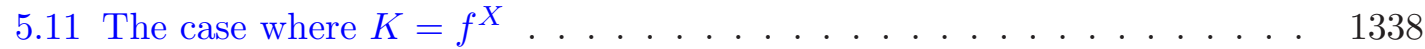

6 The analytic torsion forms of the double complex 1340

6.1 The genus $R(\theta, x) \quad \ldots \ldots \ldots \ldots \ldots$. . . . . . . . . . . . . . . . . . 1341

6.2 The holomorphic superconnection forms of the double complex . . . . . 1341

6.3 The analytic torsion forms of the double complex . . . . . . . . . . . . 1342

6.4 The case where the spectral sequence degenerates . . . . . . . . . . . . 1342

6.5 An evaluation of $T_{K, g}\left(\omega^{M}\right)$ when $K=f^{X} \ldots \ldots \ldots \ldots$. . . . . . . 1343

6.6 The de Rham superconnection forms . . . . . . . . . . . . . . 1346

6.7 Comparing the holomorphic and de Rham superconnection forms . . . . 1346

7 Rigidity, the genus $R(\theta, x)$ in degree 0 and the vanishing of $T_{K, g}\left(\omega^{M}\right)^{(0)}$

7.1 A rigid genus . . . . . . . . . . . . . . . . . . 1346

7.2 The case where $S$ is a point . . . . . . . . . . . . . . 1349

8 Morse and holomorphic instantons $\quad 1350$

8.1 Morse and Koszul localization . . . . . . . . . . . . . . . . . 1350

8.2 A complex Hermitian vector space . . . . . . . . . . . . . . . 1351

8.3 The real and holomorphic superconnections of a vector bundle . . . . 1353

References

1355

\section{Introduction}

The purpose of this paper is to establish various results on the equivariant holomorphic torsion forms associated to the de Rham complex. In particular, we show that, in general, these torsion forms vanish identically. Also we give a local formula for the equivariant holomorphic torsion forms in hypercohomology.

In fact, let $\pi: M \rightarrow S$ be a holomorphic submersion of complex manifolds with compact fibre $X$, and let $E$ be a holomorphic vector bundle on $M$. Assume that $\pi$ is Kähler, i.e. there is a closed $(1,1)$ form $\omega^{M}$ on $M$ which induces a Hermitian metric $h^{T X}$ on $T X=T M / S$. Let $h^{E}$ be a Hermitian metric on $E$. Let $G$ be a compact Lie group acting holomorphically on $M$. Assume that $G$ acts fibrewise, that the action of $G$ lifts to $E$, and that $G$ preserves $\omega^{M}$ and $h^{E}$. Finally, we assume that the $R^{i} \pi_{*} E$ are locally free. In [BGS88b, BK92, M00], if $g \in G$, Bismut, Gillet and Soulé, Bismut and Köhler, and Ma have constructed analytic torsion forms $T_{g}\left(\omega^{M}, h^{E}\right)$. Let $M_{g}$ be the fixed point manifold of $g$ in $M$, which fibres on $S$ with fibre $X_{g}$. The forms $T_{g}\left(\omega^{M}, h^{E}\right)$ are sums of $(p, p)$ forms on $S$, such that

$$
\frac{\bar{\partial} \partial}{2 i \pi} T_{g}\left(\omega^{M}, h^{E}\right)=\operatorname{ch}_{g}\left(R^{*} \pi_{*} E, h^{R^{*} \pi_{*} E}\right)-\int_{X_{g}} \operatorname{Td}_{g}\left(T X, h^{T X}\right) \operatorname{ch}_{g}\left(E, h^{E}\right) .
$$

On the right-hand side of (0.1), the characteristic classes are the ones which appear in the Lefschetz fixed point formula of Atiyah and Bott [ABo67, ABo68], evaluated in Chern-Weil theory using the corresponding holomorphic Hermitian connections. In particular the metric $h^{R^{*} \pi_{*} E}$ is a normalized version of the obvious $L_{2}$ metric on $R^{*} \pi_{*} E$, which is obtained by identifying the cohomology of the fibres to the corresponding harmonic forms. 


\section{J.-M. Bismut}

Let $T_{g}\left(\omega^{M}\right)$ be the analytic torsion forms associated to the $\mathbb{Z}$-graded vector bundle $E=\Lambda^{\bullet}\left(T^{*} X\right)$, equipped with the metric induced by $h^{T X}$. Then (0.1) gives

$$
\frac{\bar{\partial} \partial}{2 i \pi} T_{g}\left(\omega^{M}\right)=0
$$

The anomaly formulas of [BK92] show that, modulo smooth forms of the type $\bar{\partial} \alpha+\partial \beta$, $T_{g}\left(\omega^{M}\right)$ does not depend on $\omega^{M}$. If $S$ is compact and Kähler, $T_{g}\left(\omega^{M}\right)$ defines a cohomology class on $S$.

One purpose of this paper is to prove that the form $T_{g}\left(\omega^{M}\right)$ is closed, and that its cohomology class vanishes identically. This is clear for the component of degree 0 , since it coincides with the Ray-Singer analytic torsion [RS71] of the de Rham complex of the fibre, which is known to vanish. The vanishing of the higher analytic torsion forms is subtler. We obtain this vanishing in Theorem 4.15, by comparing the holomorphic torsion forms to other forms in relative de Rham theory, which were considered by Bismut and Lott [BLo95].

To put the above argument in perspective, let us briefly recall that the Levi-Civita superconnection was introduced in [B86] to establish a local version of the families index theorem of Atiyah and Singer [AS71]. Different versions of the Levi-Civita superconnection appear in two unrelated contexts: in complex geometry, in the proof given in [BGS88b] of a refined version of the Riemann-Roch-Grothendieck theorem, and also in the proof by Bismut and Lott [BLo95] of a Riemann-Roch-Grothendieck theorem for flat vector bundles. In $\S 3$ of the present paper, we show that these two versions of the Levi-Civita superconnection are related to each other by an explicit conjugation involving the Lefschetz operators $L, \Lambda, N$ of Hodge theory. To illustrate this point, let us just recall that if $X$ is Kähler,

$$
\left(\bar{\partial}^{X}+\bar{\partial}^{X *}-i\left(\partial^{X}+\partial^{X *}\right)\right)^{2}=0 .
$$

From the point of view of the present paper, an explanation for $(0.3)$ is that

$$
\bar{\partial}^{X}+\bar{\partial}^{X *}-i\left(\partial^{X}+\partial^{X *}\right)
$$

is conjugate to the de Rham operator $d^{X}$ by a conjugation involving the Lefschetz operators. When applied to families, this conjugation argument allows us to prove that the cohomology class of $T_{g}\left(\omega^{M}\right)$ vanishes.

Let now $K$ be a holomorphic section of $T X$. One can then consider the fibrewise double complex which computes the hypercohomology of the fibre. A second purpose of this paper is to evaluate the analytic torsion forms $T_{K, g}\left(\omega^{M}\right)$ of this complex using the results of Bismut and Lebeau [BL91], Bismut [B95, B97] and Ma [M00]. If the fibres $X$ are connected, if the vanishing locus $N$ of $K$ is smooth and fibres on $S$ with non-empty fibre $Y$, a result of Carrell and Liebermann [CaL73] asserts that the fibrewise spectral sequence in hypercohomology degenerates at $E_{1}$. When $K_{\mathbb{R}}$ is Killing, we give in Theorem 6.6 a formula for $T_{K, g}\left(\omega^{M}\right)$ as the sum of a local expression and of a Bott-Chern class associated to the hypercohomology of the fibre. In the local term, the genus $R(\theta, x)$ of [B94], which is an equivariant version of the $R$ genus of Gillet and Soulé [GS91], appears. Combining the degeneracy of the spectral sequence with this local formula leads to non-trivial results on the genus $R(\theta, x)$.

A third purpose of this paper is to give a partial direct explanation for this behaviour of the genus $R(\theta, x)$. In $\S 7$, we give a direct proof of the properties of the genus $R(\theta, x)$, by using the rigidity of the genera which are associated to the locally trivial action of $G$ on the $H^{(p, q)}(X, \mathbb{C})$.

This paper is organized as follows. In $\S 1$, we establish simple identities which relate the natural representations of the Clifford algebra of a complex Hermitian vector space. The operators $L, \Lambda, N$ appear in these identities. 


\section{HOLOMORPHIC AND DE RHAM TORSION}

In $\S 2$, we recall various differential geometric properties of Kähler fibrations in the sense of [BGS88b] and of the corresponding Gauss-Manin connection on the direct image of the constant sheaf, and on the corresponding Hodge filtration.

In $\S 3$, we compare the associated holomorphic and real Levi-Civita superconnections. More precisely, we give an explicit conjugation formula relating the two superconnections.

In $\S 4$, we prove the vanishing of the holomorphic analytic torsion forms $T_{g}\left(\omega^{M}\right)$.

In $\S 5$, if $K$ is a holomorphic section of $T X$, we derive various properties of the corresponding hypercohomology, and we establish a conjugation formula relating corresponding Levi-Civita superconnections.

In $\S 6$, we give a formula for the equivariant holomorphic analytic torsion forms in hypercohomology. Also, we prove they are locally constant on $G$.

In $\S 7$, we prove that these torsion forms vanish in degree 0 using rigidity arguments.

Finally, in $\S 8$, we derive various consequences of the conjugacy formulas of $\S 5$. In particular, we show that the Morse instantons considered by Witten [W82] and also corresponding instantons for the hypercohomology are in fact conjugate to each other. Also we relate the genus $R(\theta, x)$ to a corresponding genus $J(\theta, x)$ constructed by Bismut and Goette [BGo01] in de Rham theory. These two genera, which are formally closely related, appear as deriving from essentially similar constructions, where the roles of the Hodge operators are simply interchanged.

In the whole paper, if $\mathcal{A}$ is a $\mathbb{Z}_{2}$-graded algebra, and if $A, B \in \mathcal{A},[A, B]$ denotes the supercommutator of $A$ and $B$. If $\mathcal{A}, \mathcal{B}$ are $\mathbb{Z}_{2}$-graded algebras, $\mathcal{A} \widehat{\otimes} \mathcal{B}$ is the $\mathbb{Z}_{2}$-graded algebra which is the tensor product of $\mathcal{A}$ and $\mathcal{B}$. If $V$ is a $\mathbb{Z}_{2}$-graded finite-dimensional vector space, if $A \in \operatorname{End}(V), \operatorname{Tr}_{\mathrm{s}}[A] \in \mathbb{C}$ is the supertrace of $A$. If $\mathcal{A}$ is supercommutative, we extend $\operatorname{Tr}_{\mathrm{s}}$ to a linear map from $\mathcal{A} \widehat{\otimes} \operatorname{End}(V)$ into $\mathcal{A}$. It is an elementary fact [Q85] that the supertrace of a supercommutator vanishes.

\section{Clifford algebras and exterior algebras}

In this section, we collect a few elementary facts on Clifford algebras. In particular, we give an explicit formula relating the action of a Clifford algebra on real and complex versions of the exterior algebra, in which the operators $L, \Lambda, N$ appear explicitly.

This section is organized as follows. In $\S 1$, we introduce the operators $L, \Lambda, N$. In $\S 1.2$, we recall the definition of a Clifford algebra. Finally, in $\S 1.3$, we relate real and complex representations of a Clifford algebra.

\subsection{A symplectic vector space}

Let $V$ be a real vector space of even dimension $n=2 \ell$. Let $\omega$ be a real symplectic form on $V$, i.e. a non-degenerate element of $\Lambda^{2}\left(V^{*}\right)$. Let $L \in \operatorname{End}\left(\Lambda^{*}\left(V^{*}\right)\right)$ be given by

$$
L \alpha=\omega \wedge \alpha .
$$

Let $\Lambda \in \operatorname{End}\left(\Lambda^{\bullet}(V)\right)$ be the transpose of $L$.

Let $e_{1}, \ldots, e_{n}$ be a basis of $V$, and let $e^{1}, \ldots, e^{n}$ be the corresponding dual basis of $V^{*}$. Then

$$
L=\frac{1}{2} \omega\left(e_{i}, e_{j}\right) e^{i} \wedge e^{j}, \quad \Lambda=-\frac{1}{2} \omega\left(e_{i}, e_{j}\right) i_{e^{i}} i_{e^{j}} .
$$

Let $\phi: V \rightarrow V^{*}$ be the isomorphism canonically associated to $\omega$, so that if $X, Y \in V$,

$$
\omega(X, Y)=\langle X, \phi Y\rangle .
$$

In the sequel, we will identify $V$ and $V^{*}$ by the map $\phi$. Let $\omega^{*}$ be the corresponding symplectic form on $V^{*}$. 


\section{J.-M. Bismut}

Also we identify $\Lambda^{\bullet}(V)$ and $\Lambda^{\bullet}\left(V^{*}\right)$ by $\phi$. The operator $\Lambda$ now acts on $\Lambda^{\bullet}\left(V^{*}\right)$, and this action is given by the formula

$$
\Lambda=-\frac{1}{2} \omega^{*}\left(e^{i}, e^{j}\right) i_{e_{i}} i_{e_{j}} .
$$

Let $N$ be the number operator of $\Lambda^{*}\left(V^{*}\right)$, which acts on $\Lambda^{k}\left(V^{*}\right)$ by multiplication by $k$. Put

$$
H=\frac{1}{2}\left(N-\frac{n}{2}\right) \text {. }
$$

Then we have the classical $s l_{2}$ commutation relations,

$$
[H, L]=L, \quad[H, \Lambda]=-\Lambda, \quad[L, \Lambda]=2 H .
$$

\subsection{Clifford algebras and exterior algebras}

Let $\langle$,$\rangle be a Euclidean product on V$. Let $c(V)$ be the Clifford algebra of $(V,\langle\rangle)$, i.e. the algebra spanned over $\mathbb{R}$ by $1, X \in V$, and the commutation relations

$$
X Y+Y X=-2\langle X, Y\rangle .
$$

Then $c(V)$ is canonically filtered by the length (which defines an increasing filtration), and the corresponding $\mathrm{Gr}$ is just the exterior algebra $\Lambda^{\bullet}(V)$. Also there is a canonical isomorphism of $c(V)$ and $\Lambda^{\bullet}(V)$ as vector spaces.

If $X \in V$, let $X^{*} \in V^{*}$ correspond to $X$ by the scalar product. Set

$$
c(X)=X^{*}-i_{X}, \quad \widehat{c}(X)=X^{*} \wedge+i_{X} .
$$

Then $c(X), \widehat{c}(X)$ are odd operators acting on $\Lambda^{\bullet}\left(V^{*}\right)$. If $X, Y \in V$,

$$
[c(X), c(Y)]=-2\langle X, Y\rangle, \quad[\widehat{c}(X), \widehat{c}(Y)]=2\langle X, Y\rangle, \quad[c(X), \widehat{c}(Y)]=0 .
$$

Under the isomorphism of vector spaces $c(V) \sim \Lambda^{\bullet}\left(V^{*}\right)$, one verifies easily that if $c(X)$ corresponds to left multiplication by $X$ on $c(V),(-1)^{N} \widehat{c}(X)$ corresponds to right multiplication by $X$ on $c(V)$.

If $A \in \operatorname{End}(V)$, then $A$ acts naturally on $\Lambda^{*}\left(V^{*}\right)$ by the formula

$$
A=-\left\langle A e_{i}, e^{j}\right\rangle e^{i} i_{e_{j}}
$$

Assume now that $\mathrm{A}$ is antisymmetric. If $e_{1}, \ldots e_{n}$ is an orthonormal basis of $V$, then

$$
A=\frac{1}{4}\left\langle A e_{i}, e_{j}\right\rangle\left(c\left(e_{i}\right) c\left(e_{j}\right)-\widehat{c}\left(e_{i}\right) \widehat{c}\left(e_{j}\right)\right) .
$$

\subsection{Complex structures and exterior algebras}

Let $J \in \operatorname{End}(V)$ be a complex structure on $V$, so that $J^{2}=-1$. Let $W, \bar{W}$ be the $\pm i$ eigenspaces of $J$ in $V \otimes_{\mathbb{R}} \mathbb{C}$. Then

$$
V \otimes_{\mathbb{R}} \mathbb{C}=W \oplus \bar{W} .
$$

We can identify $W$ and $V$ as real vector spaces equipped with a complex structure, the identification being given by $X \in W \rightarrow X+\bar{X} \in V$.

We will assume that $\omega$ is $J$-invariant and also that $J$ polarizes $\omega$. Namely there is a $J$-invariant Euclidean product $\langle$,$\rangle on V$ such that if $X, Y \in V$,

$$
\omega(X, Y)=\langle X, J Y\rangle .
$$

Then $J$ is antisymmetric with respect to $\langle$,$\rangle . If V$ and $V^{*}$ are identified by the scalar product $\langle$,$\rangle ,$ the map $\phi: V \rightarrow V^{*}$ defined in (1.3) is just given by $J$. Also $\omega$ and $\omega^{*}$ coincide.

Assume that $e_{1}, \ldots, e_{n}$ is an orthonormal basis of $V$. By (1.4), the operator $\Lambda$ is now given by the formula

$$
\Lambda=-\frac{1}{2} \omega\left(e_{i}, e_{j}\right) i_{e_{i}} i_{e_{j}}
$$




\section{HOLOMORPHIC AND DE RHAM TORSION}

Finally observe that $\Lambda$ is the adjoint of $L$ with respect to the obvious scalar product on $\Lambda^{\bullet}\left(V^{*}\right)$ induced by $\langle$,$\rangle .$

We still denote by $\langle$,$\rangle the associated Hermitian product on W$. Clearly,

$$
\Lambda^{\bullet}\left(V^{*}\right) \otimes_{\mathbb{R}} \mathbb{C} \sim \Lambda^{\bullet}\left(\bar{W}^{*}\right) \widehat{\otimes} \Lambda^{\bullet}\left(W^{*}\right) .
$$

If $X \in V$, we still denote by $X^{*}$ the corresponding element in $V^{*}$. If $X \in W$, then $X^{*} \in \bar{W}^{*}$, and if $X \in \bar{W}$, then $X^{*} \in W^{*}$.

We will use the notation of $\S 1.2$ with respect to the given Euclidean product $\langle$,$\rangle . The exterior$ algebras $\Lambda^{\bullet}\left(\bar{W}^{*}\right)$ and $\Lambda^{\bullet}\left(W^{*}\right)$ are $c(V)$-modules. Indeed, if $X \in W, Y \in \bar{W}$, set

$$
\begin{gathered}
c_{\bar{W}}(X)=\sqrt{2} X^{*} \wedge, \quad c_{\bar{W}}(Y)=-\sqrt{2} i_{Y}, \\
c_{W}(X)=-\sqrt{2} i_{X}, \quad c_{W}(Y)=\sqrt{2} Y^{*} \wedge .
\end{gathered}
$$

Of course the action of the operators in (1.16) can be extended to $\Lambda^{\bullet}\left(V^{*}\right) \otimes_{\mathbb{R}} \mathbb{C} \simeq \Lambda^{\bullet}\left(\bar{W}^{*}\right) \widehat{\otimes} \Lambda^{\bullet}\left(W^{*}\right)$. Then if $X, Y \in V \otimes_{\mathbb{R}} \mathbb{C}$,

$$
\left[c_{\bar{W}}(X), c_{\bar{W}}(Y)\right]=-2\langle X, Y\rangle, \quad\left[c_{W}(X), c_{W}(Y)\right]=-2\langle X, Y\rangle, \quad\left[c_{\bar{W}}(X), c_{W}(Y)\right]=0 .
$$

Moreover one verifies easily that if $X \in V$,

$$
c(X)=\frac{1}{\sqrt{2}}\left(c_{W}(X)+c_{\bar{W}}(X)\right), \quad \widehat{c}(X)=\frac{i}{\sqrt{2}}\left(c_{W}(J X)-c_{\bar{W}}(J X)\right) .
$$

Note that the commutation relations in (1.9) follow from (1.17) and (1.18).

Let $w_{1}, \ldots, w_{\ell}$ be an orthonormal basis of $W$, and let $w^{1}, \ldots, w^{\ell}$ be the corresponding dual basis of $W^{*}$. Then

$$
L=-\sqrt{-1} w^{i} \wedge \bar{w}^{i}, \quad \Lambda=\sqrt{-1} i_{\bar{w}_{i}} i_{w_{i}}
$$

DEFINITION 1.1. Set

$$
M=L-\Lambda, \quad R=L+\Lambda .
$$

Then $M$ is antisymmetric in $\operatorname{End}\left(\Lambda^{*}\left(V^{*}\right)\right)$, and $R$ is symmetric.

Proposition 1.2. If $X \in V$,

$$
\left[M, i_{X}\right]=(J X)^{*} \wedge, \quad\left[M, X^{*} \wedge\right]=i_{J X}
$$

If $X \in V, \vartheta \in \mathbb{R}$,

$$
\begin{gathered}
e^{\vartheta M} i_{X} e^{-\theta M}=\cos (\vartheta) i_{X}+\sin (\vartheta)(J X)^{*} \wedge, \\
e^{\vartheta M} X^{*} \wedge e^{-\vartheta M}=\cos (\vartheta) X^{*} \wedge+\sin (\vartheta) i_{J X} .
\end{gathered}
$$

Proof. The identities in (1.21) are obvious consequences of (1.2), (1.13) and (1.14). From (1.21), we get (1.22).

Remark 1.3. Observe that if $X \in V, Y \in V^{*}$, using (1.21), we can rewrite (1.22) in the form

$$
\begin{gathered}
e^{\vartheta M} i_{X} e^{-\vartheta M}=\cos (\vartheta) i_{X}-\sin (\vartheta) i_{X} \omega, \\
e^{\vartheta M} Y \wedge e^{-\vartheta M}=\cos (\theta) Y \wedge+\sin (\theta)[Y \wedge, \Lambda] .
\end{gathered}
$$

We emphasize the fact that (1.23) does not depend on the choice of $J$, while (1.21) seems to depend on this choice.

Let $N^{(0,1)}, N^{(1,0)}$ be the number operators on $\Lambda^{\bullet}\left(\bar{W}^{*}\right), \Lambda^{\bullet}\left(W^{*}\right)$ so that

$$
N=N^{(0,1)}+N^{(1,0)} \text {. }
$$


Set

$$
\mathcal{N}=N^{(0,1)}-N^{(1,0)}
$$

Proposition 1.4. If $X \in V, \vartheta \in \mathbb{R}$, then

$$
\begin{gathered}
e^{\vartheta M} c_{\bar{W}}(X) e^{-\vartheta M}=\cos (\vartheta) c_{\bar{W}}(X)-\sin (\vartheta) c_{W}(J X), \\
e^{\vartheta M} c_{W}(X) e^{-\vartheta M}=\cos (\vartheta) c_{W}(X)-\sin (\vartheta) c_{\bar{W}}(J X), \\
e^{\vartheta M} c(X) e^{-\vartheta M}=\cos (\vartheta) c(X)-\sin (\vartheta) c(J X), \\
e^{\vartheta M} \widehat{c}(X) e^{-\vartheta M}=\cos (\vartheta) \widehat{c}(X)+\sin (\vartheta) \widehat{c}(J X), \\
e^{\vartheta M} N^{(0,1)} e^{-\vartheta M}=N^{(0,1)}-\frac{\sin (2 \vartheta)}{2} R+(\cos (2 \vartheta)-1) H, \\
e^{\vartheta M} N^{(1,0)} e^{-\vartheta M}=N^{(1,0)}-\frac{\sin (2 \vartheta)}{2} R+(\cos (2 \vartheta)-1) H, \\
e^{\vartheta M} L e^{-\vartheta M}=L+\sin (2 \vartheta) H+(\cos (2 \vartheta)-1) \frac{R}{2} .
\end{gathered}
$$

Moreover,

$$
\begin{aligned}
& i^{-N^{(1,0)}} e^{\vartheta M} c_{\bar{W}}(X) e^{-\vartheta M} i^{N^{(1,0)}}=\cos (\vartheta) c_{\bar{W}}(X)+\sin (\vartheta) c_{W}(X), \\
& i^{N^{(0,1)}} e^{\vartheta M} c_{W}(X) e^{-\vartheta M} i^{-N^{(0,1)}}=\cos (\vartheta) c_{W}(X)+\sin (\vartheta) c_{\bar{W}}(X) .
\end{aligned}
$$

Proof. The first four identities in (1.26) follow from Proposition 1.2. Moreover using (1.6), we get

$$
\left[M, N^{(0,1)}\right]=-R, \quad[M, R]=4 H, \quad[M, H]=-R .
$$

From (1.28), we get the last three identities in (1.26). The identities in (1.27) follow from (1.26).

THEOREM 1.5. If $X \in V$, then

$$
\begin{gathered}
i^{-N^{(1,0)}} e^{\pi M / 4} c_{\bar{W}}(X) e^{-\pi M / 4} i^{N^{(1,0)}}=c(X), \\
i^{N^{(0,1)}} e^{\pi M / 4} c_{W}(X) e^{-\pi M / 4} i^{-N^{(0,1)}}=c(X), \\
i^{-N^{(1,0)}} e^{\pi M / 4} c_{W}(X) e^{-\pi M / 4} i^{N^{(1,0)}}=-i \widehat{c}(X), \\
i^{-N^{(1,0)}} e^{\pi / 4 M} N^{(0,1)} e^{-\pi / 4 M} i^{N^{(1,0)}}=\frac{1}{2} \mathcal{N}+\frac{n}{4}+\frac{i}{2} M, \\
i^{-N^{(1,0)}} e^{\pi M / 4} N^{(1,0)} e^{-\pi M / 4} i^{N^{(1,0)}}=-\frac{1}{2} \mathcal{N}+\frac{n}{4}+\frac{i}{2} M, \\
i^{-N^{(1,0)}} e^{\pi M / 4}\left(N-\frac{n}{2}-M\right) e^{-\pi M / 4} i^{N^{(1,0)}}=2 i L .
\end{gathered}
$$

Proof. Our theorem is a special case of Proposition 1.4.

Remark 1.6. By Theorem 1.5, we have found that the Clifford actions $c_{\bar{W}}$ and $c_{W}$ on $\Lambda^{\bullet}\left(V^{*}\right) \otimes_{\mathbb{R}} \mathbb{C}$ are conjugate to the standard Clifford action $c$ by an explicit conjugation. Moreover, since

$$
\left[c_{\bar{W}}(X), N^{(1,0)}\right]=0
$$

we deduce from (1.29) the identity

$$
\left[c(X),-\frac{1}{2} \mathcal{N}+\frac{i}{2} M\right]=0 .
$$

Needless to say, this simple identity can be proved directly. 


\section{HOLOMORPHIC AND DE RHAM TORSION}

\section{Kähler fibrations and Gauss-Manin connections}

In this section, we give various differential geometric properties of Kähler fibrations in the sense of [BGS88b], and we introduce the corresponding Gauss-Manin connection on the cohomology of the fibres.

This section is organized as follows. In $\S 2.1$, we introduce the de Rham and Dolbeault complexes of a compact complex Kähler manifold $X$. In $\S 2.2$, we recall the definition of a Kähler fibration given in Bismut, Gillet and Soulé [BGS88b]. In $\S 2.3$, we give a formula for the de Rham operator of the total space $M$ of the fibration. In $\S \S 2.4$ and 2.5, we recover a result of [BGS88b] which expresses the compatibility of Riemannian to holomorphic data on a Kähler fibration. In $\S 2.6$, we give a formula for the Dolbeault operator of $M$. In $\S 2.7$ we evaluate the Gauss-Manin connection on $H^{\bullet}(X, \mathbb{R})$. Finally, in $\S 2.8$, we construct the corresponding connections on the Hodge decomposition of $H^{\bullet}(X, \mathbb{C})$.

\subsection{Dolbeault and de Rham complexes}

Let $X$ be a compact complex manifold of real dimension $n=2 \ell$. Let $T_{\mathbb{R}} X$ be the corresponding real tangent bundle, and let $J^{T_{\mathbb{R}} X} \in \operatorname{End}\left(T_{\mathbb{R}} X\right)$ be the complex structure of $X$. Let $T X, \overline{T X}$ be the holomorphic and antiholomorphic tangent bundles, i.e. the $i$ and $-i$ eigenbundles of $J^{T_{\mathbb{R}} X}$ in $T_{\mathbb{R}} X \otimes_{\mathbb{R}} \mathbb{C}$. Let $\omega^{X}$ be a real closed 2 -form of type $(1,1)$ on $X$. We assume that $\omega^{X}$ is a Kähler form, i.e. there is a scalar product on $T_{\mathbb{R}} X$ such that if $A, B \in T_{\mathbb{R}} X$,

$$
\omega^{X}(A, B)=\left\langle A, J^{T_{\mathbb{R}} X} B\right\rangle .
$$

We denote by $h^{T X}$ the associated Hermitian product on $T X$.

Now we use the same notation as in $\S 1$. Namely $L$ denotes the operator of multiplication by $\omega^{X}$ acting on the bundle of algebras $\Lambda^{*}\left(T_{\mathbb{R}}^{*} X\right)$, and $\Lambda$ is its adjoint.

Let $\left(\Omega^{\bullet}(X, \mathbb{R}), d^{X}\right)$ be the de Rham complex of smooth sections of $\Lambda^{\bullet}\left(T_{\mathbb{R}}^{*} X\right)$ on $X$. Its cohomology is the real cohomology $H^{\bullet}(X, \mathbb{R})$. Note the duality between $\Omega^{m}(X, \mathbb{R})$ and $\Omega^{n-m}(X, \mathbb{R})$,

$$
s, s^{\prime} \rightarrow \frac{1}{(2 \pi)^{\ell}} \int_{X} s \wedge s^{\prime},
$$

which induces the Poincaré duality of $H^{m}(X, \mathbb{R})$ and $H^{n-m}(X, \mathbb{R})$.

Let $\Omega^{\bullet}(X, \mathbb{C})$ denote the complexification of $\Omega^{\bullet}(X, \mathbb{R})$, i.e. the vector space of smooth sections of $\Lambda^{\bullet}\left(T_{\mathbb{R}}^{*} X\right) \otimes_{\mathbb{R}} \mathbb{C}$. Then $\Omega^{\bullet}(X, \mathbb{C})$ splits as

$$
\Omega^{\bullet}(X, \mathbb{C})=\bigoplus_{0 \leqslant p, q \leqslant \ell} \Omega^{(p, q)}(X, \mathbb{C})
$$

where $\Omega^{(p, q)}(X, \mathbb{C})$ is the vector space of smooth sections of $\Lambda^{p}\left(T^{*} X\right) \widehat{\otimes} \Lambda^{q}\left(\overline{T^{*} X}\right)$. The operator $d^{X}$ splits as

$$
d^{X}=\bar{\partial}^{X}+\partial^{X}
$$

Let $*$ be the star operator acting on $\Lambda^{*}\left(T_{\mathbb{R}}^{*} X\right)$ which is associated to the given metric on $T_{\mathbb{R}} X$. We equip $\Omega^{\bullet}(X, \mathbb{R})$ with the scalar product $h^{\Omega^{*}(X, \mathbb{R})}$ given by

$$
s, s^{\prime} \in \Omega^{\bullet}(X, \mathbb{R}) \rightarrow\left\langle s, s^{\prime}\right\rangle=\frac{1}{(2 \pi)^{\ell}} \int_{X} s \wedge * s^{\prime} .
$$

We extend (2.5) to a Hermitian product on $\Omega^{\bullet}(X, \mathbb{C})$. Then the $\Omega^{(p, q)}(X, \mathbb{C})$ are mutually orthogonal in $\Omega^{*}(X, \mathbb{C})$. Let $d^{X, *}$ be the formal adjoint of $d^{X}$ with respect to $(2.5)$. Then $d^{X, *}$ splits as

$$
d^{X, *}=\bar{\partial}^{X, *}+\partial^{X, *}
$$


Moreover since $X$ is Kähler, by [GrHa78, p. 111],

$$
\bar{\partial}^{X, *}=-i\left[\partial^{X}, \Lambda\right], \quad \partial^{X, *}=i\left[\bar{\partial}^{X}, \Lambda\right] .
$$

Note that our signs differ from the signs in [GrHa78], because we chose a different sign convention for the Kähler form $\omega^{X}$.

Using the fact that $\left[\bar{\partial}^{X}, \partial^{X}\right]=0$, we deduce from (2.7) that

$$
\begin{gathered}
{\left[\bar{\partial}^{X}, \partial^{X, *}\right]=0,\left[\partial^{X}, \bar{\partial}^{X, *}\right]=0,} \\
{\left[\bar{\partial}^{X}, \bar{\partial}^{X, *}\right]=\left[\partial^{X}, \partial^{X, *}\right]=i\left[\partial^{X},\left[\bar{\partial}^{X}, \Lambda\right]\right] .}
\end{gathered}
$$

From (2.8), we obtain the well-known equality in [GrHa78, p. 115],

$$
\left[d^{X}, d^{X, *}\right]=2\left[\bar{\partial}^{X}, \bar{\partial}^{X, *}\right]=2\left[\partial^{X}, \partial^{X, *}\right]
$$

Let $F^{\bullet}$ be the Hodge decreasing filtration on $\Omega^{\bullet}(X, \mathbb{C})$, i.e. for $p \in \mathbb{N}$, set

$$
F^{p} \Omega^{\bullet}(X, \mathbb{C})=\bigoplus_{p \leqslant p^{\prime}, 0 \leqslant q \leqslant \ell} \Omega^{\left(p^{\prime}, q\right)}(X, \mathbb{C}) .
$$

The corresponding $\operatorname{Gr}^{\bullet} \Omega^{\bullet}(X, \mathbb{C})$ is given by

$$
\operatorname{Gr}^{p} \Omega^{\bullet}(X, \mathbb{C})=F^{p} \Omega^{\bullet}(X, \mathbb{C}) / F^{p+1} \Omega^{\bullet}(X, \mathbb{C}) .
$$

Then $\operatorname{Gr}^{p} \Omega^{\bullet}(X, \mathbb{C})$ is a $\mathbb{Z}$-graded vector space given by

$$
\operatorname{Gr}^{p} \Omega^{\bullet}(X, \mathbb{C})=\bigoplus_{0 \leqslant q \leqslant \ell} \Omega^{(p, q)}(X, \mathbb{C}) .
$$

From (2.9), we find that the Hodge spectral sequence degenerates, and moreover, we have the Hodge decomposition

$$
H^{m}(X, \mathbb{C})=\bigoplus_{p+q=m} H^{(p, q)}(X)
$$

The filtration $F^{\bullet}$ induces a corresponding filtration on $H^{\bullet}(X)$.

\subsection{A Kähler fibration}

Let $\pi: M \rightarrow S$ be a holomorphic submersion of complex manifolds, with compact fibre $X$. Let $T_{\mathbb{R}} X=T_{\mathbb{R}} M / S$ be the fibrewise real tangent bundle to the fibres $X$, and let $T X=T M / S$ be the corresponding fibrewise holomorphic tangent bundle. Let $J^{T_{\mathbb{R}}} X$ be the complex structure on $T_{\mathbb{R}} X$.

Let $T^{H} M$ be a smooth subbundle of $T M$ so that

$$
T M=T^{H} M \oplus T X .
$$

Let $h^{T X}$ be a Hermitian metric on $T X$.

Now we follow [BGS88b, Definition 1.4] and [B97, ch. 2].

Definition 2.1. The triple $\left(\pi, h^{T X}, T^{H} M\right)$ is said to define a Kähler fibration if there exists a smooth real 2-form $\omega^{M}$ of complex type $(1,1)$ on $M$ which has the following properties:

a) $\omega^{M}$ is closed.

b) $T_{\mathbb{R}}^{H} M$ and $T_{\mathbb{R}} X$ are orthogonal with respect to $\omega^{M}$.

c) If $A, B \in T_{\mathbb{R}} X$ then

$$
\omega^{M}(A, B)=\left\langle A, J^{T_{\mathbb{R}} X} B\right\rangle .
$$

From (2.15) we deduce that the metric $h^{T X}$ is fibrewise Kähler, and that the restriction of $\omega^{M}$ to $T_{\mathbb{R}} X$ is the associated Kähler metric of the fibres. 


\section{HolOMORPHIC AND DE RHAM TORSION}

By [BGS88b, Theorems 1.5 and 1.7], if $\omega^{M}$ is a real closed 2-form on $M$ of type $(1,1)$, which induces a Kähler metric $h^{T X}$ along the fibres $X$, and if $T^{H} M$ denotes the orthogonal bundle to $T X$ in $T M$ with respect to $\omega^{M}$, then $\left(\pi, h^{T X}, T^{H} M\right)$ is a Kähler fibration, and $\omega^{M}$ is an associated $(1,1)$ form. Moreover if $\omega^{M \prime}$ is another closed real $(1,1)$ form on $M$ which is associated to $\left(\pi, h^{T X}, T^{H} M\right)$, there exists a closed real $(1,1)$ form $\eta$ on $S$ such that

$$
\omega^{M \prime}-\omega^{M}=\pi^{*} \eta \text {. }
$$

Now we will assume that $\left(\pi, h^{T X}, T^{H} M\right)$ is a Kähler fibration and that $\omega^{M}$ is an associated $(1,1)$ form. Let $P^{T X}: T_{\mathbb{R}} M \rightarrow T_{\mathbb{R}} X$ be the obvious projection with respect to the splitting (2.14). By (2.14), we find that

$$
\Lambda^{\bullet}\left(T_{\mathbb{R}}^{*} M\right) \simeq \pi^{*} \Lambda^{\bullet}\left(T_{\mathbb{R}}^{*} S\right) \widehat{\otimes} \Lambda^{\bullet}\left(T_{\mathbb{R}}^{*} X\right),
$$

and that $(2.17)$ induces an identification of the corresponding $(p, q)$ decompositions.

Let $\omega^{X}, \omega^{H}$ be the restrictions of $\omega^{M}$ to $T_{\mathbb{R}} X, T_{\mathbb{R}}^{H} M$. Using $(2.17)$, we see that $\omega^{X}, \omega^{H}$ can be considered as $(1,1)$ forms on $M$, and that

$$
\omega^{M}=\omega^{X}+\omega^{H} .
$$

Moreover $\omega^{X}$ is the Kähler form of the fibres $X$.

\subsection{The de Rham operator of $M$}

If $A \in T_{\mathbb{R}} S$, let $A^{H} \in T_{\mathbb{R}}^{H} M$ be the horizontal lift of $A$. If $A, B \in T_{\mathbb{R}} S$, set

$$
T^{H}(A, B)=-P^{T X}\left[A^{H}, B^{H}\right] .
$$

Then $T^{H}$ is a horizontal tensor with values in $T_{\mathbb{R}} X$.

Now we use the same notation as in $(2.3)$. Then $\Omega^{\bullet}(X, \mathbb{R}), \Omega^{\bullet}(X, \mathbb{C})$ and the $\Omega^{(p, q)}(X, \mathbb{C})$ are infinite-dimensional vector bundles on $S$. By $(2.17)$,

$$
\Omega^{\bullet}(M, \mathbb{R})=\Omega^{\bullet}\left(S, \Omega^{\bullet}(X, \mathbb{R})\right) .
$$

By (2.20), the action of the fibrewise de Rham operator $d^{X}$ extends to $\Omega^{\bullet}(M, \mathbb{R})$.

If $A$ is a smooth section of $T_{\mathbb{R}} S$, the Lie derivative operator $L_{A^{H}}$ acts naturally on $\Omega^{\bullet}(X, \mathbb{R})$, and the action is tensorial in $A$.

Definition 2.2. If $A \in T_{\mathbb{R}} S$, and if $s$ is a smooth section of $\Omega^{\bullet}(X, \mathbb{R})$, set

$$
\nabla_{U}^{\Omega^{\bullet}(X, \mathbb{R})} s=L_{A^{H}} s .
$$

Then one verifies easily that $\nabla^{\Omega^{\bullet}(X, \mathbb{R})}$ is a connection on the $\mathbb{Z}$-graded vector bundle $\Omega^{\bullet}(X, \mathbb{R})$, which is compatible with the Poincaré duality in $(2.2)$. Note that in general, $\nabla^{\Omega^{*}(X, \mathbb{R})}$ does not preserve the splitting $(2.3)$ of $\Omega^{*}(X, \mathbb{R})$. Combining with exterior differentiation on $\Omega^{*}(S, \mathbb{R})$, the action of $\nabla^{\Omega^{\bullet}(X, \mathbb{R})}$ extends to an action on $\Omega^{\bullet}\left(S, \Omega^{\bullet}(X, \mathbb{R})\right) \sim \Omega^{\bullet}(M, \mathbb{R})$.

Finally the interior multiplication operator $i_{T^{H}}$ acts on $\Omega^{\bullet}(M, \mathbb{R})$. Now we have a classical result, stated in [BLo95, Proposition 3.4].

Proposition 2.3. The following identity of operators acting on $\Omega^{\bullet}(M, \mathbb{R})$ holds:

$$
d^{M}=d^{X}+\nabla^{\Omega^{\bullet}(X, \mathbb{R})}+i_{T^{H}}
$$

Remark 2.4. Note that $L_{T^{H}}$ is a 2 -form on $S$ with values in operators acting along the fibres. Since $d^{M, 2}=0$, from (2.16), we get

$$
\begin{gathered}
d^{X, 2}=0, \quad\left[\nabla^{\Omega^{\bullet}(X, \mathbb{R})}, d^{X}\right]=0, \quad \nabla^{\Omega^{\bullet}(X, \mathbb{R}), 2}+L_{T^{H}}=0, \\
{\left[\nabla^{\Omega^{\bullet}(X, \mathbb{R})}, i_{T^{H}}\right]=0, \quad i_{T^{H}}^{2}=0 .}
\end{gathered}
$$




\section{J.-M. Bismut}

\subsection{The holomorphic Hermitian connection on $T^{*} X$}

Note that the form $\omega^{X}$ is symplectic along the fibres $X$. Recall that if $\mathcal{H}: M \rightarrow \mathbb{R}$ is a smooth function, the fibrewise Hamiltonian vector field $X^{\mathcal{H}}$ is such that

$$
d^{X} \mathcal{H}+i_{X} \mathcal{H} \omega^{X}=0 \text {. }
$$

Let $J^{T_{\mathbb{R}}^{*} X}$ be the complex structure of $T_{\mathbb{R}}^{*} X$. Then $T^{*} X, \overline{T^{*} X}$ are the $i,-i$ bundles of $J^{T_{\mathbb{R}}^{*}} X$ in $T_{\mathbb{R}}^{*} X \otimes_{\mathbb{R}} \mathbb{C}$. Let $h^{T^{*} X}$ be the Hermitian metric on $T^{*} X$ associated to $h^{T X}$, and let $\omega^{X *}$ be the section of $\Lambda^{2}\left(T_{\mathbb{R}} X\right)$ such that, if $U, V \in T_{\mathbb{R}}^{*} X$,

$$
\omega^{X *}(U, V)=-\left\langle U, J^{T_{\mathbb{R}}^{*} X} V\right\rangle .
$$

Then $\omega^{X *}$ is a symplectic form on the fibres of $T_{\mathbb{R}}^{*} X$.

TheOREM 2.5. The following identities hold:

$$
\begin{gathered}
d^{X} \omega^{X}=0, \quad \nabla^{\Omega^{\bullet}(X, \mathbb{R})} \omega^{X}=0, \\
d^{X} \omega^{H}+i_{T^{H}} \omega^{X}=0, \quad \nabla^{\Omega^{*}(X, \mathbb{R})} \omega^{H}=0 .
\end{gathered}
$$

The connection $\nabla^{\Omega^{\bullet}(X, \mathbb{R})}$ preserves the fibrewise symplectic form $\omega^{X}$. In particular, if $A, B \in$ $T_{\mathbb{R}} S, T^{H}(A, B)$ is the fibrewise Hamiltonian vector field associated to the fibrewise Hamiltonian $\omega^{H}\left(A^{H}, B^{H}\right)$. The tensor $T^{H}$ is of type $(1,1)$. If $s$ is a smooth section of $T^{*} X$, and if $A \in T S$, then $L_{\bar{A}^{H}} S$ is the section of $T^{*} X$ given by

$$
L_{\bar{A}^{H}} s=\nabla_{\bar{A}^{H}}^{T^{*} X^{\prime \prime}} s .
$$

If $A \in T_{\mathbb{R}} S$, then $L_{A^{H}} J^{T_{\mathbb{R}}^{*} X}$ and $J^{T_{\mathbb{R}}^{*} X} L_{A^{H}} J^{T_{\mathbb{R}}^{*} X}$ are symmetric tensors anticommuting with $J^{T_{\mathbb{R}}^{*} X}$. Moreover if $B \in T S, L_{\bar{B}^{H}} J^{T_{\mathbb{R}}^{*} X}$ vanishes on $T^{*} X$ and maps $\overline{T^{*} X}$ into $T^{*} X$. Finally, if $A \in T_{\mathbb{R}} S$,

$$
\left(h^{T_{\mathbb{R}}^{*} X}\right)^{-1} L_{A^{H}} h^{T_{\mathbb{R}}^{*} X}=-J^{T_{\mathbb{R}}^{*} X} L_{A^{H}} J^{T_{\mathbb{R}}^{*} X} .
$$

Proof. Using (2.18) and Proposition 2.3 and splitting the equation for $d^{M} \omega$ into its various components, we get (2.26). The second equation in (2.26) just says that $\nabla^{\Omega^{\bullet}(X, \mathbb{R})}$ preserves $\omega^{X}$. Since $\omega^{H}$ is of complex type $(1,1)$, from the third equation in $(2.26)$, we find that $T^{H}$ is of type $(1,1)$. If $s$ is a smooth section of $T^{*} X$ and if $A$ is a smooth section of $T S$, then

$$
L_{\bar{A}^{H}} s=\left(d^{M} i_{\bar{A}^{H}}+i_{\bar{A}^{H}} d^{M}\right) s .
$$

Equivalently we can rewrite (2.29) as

$$
L_{\bar{A}^{H}} s=i_{\bar{A}^{H}} \bar{\partial}^{M} s,
$$

which is just (2.27).

Observe that the map $U \in T_{\mathbb{R}} X \rightarrow-i_{U} \omega^{X} \in T_{\mathbb{R}}^{*} X$ is an identification of smooth vector bundles. Moreover the form $\omega^{X, *}$ on $T_{\mathbb{R}}^{*} X$ is obtained from $\omega^{X}$ via this identification. Using (2.26), we find that if $A \in T_{\mathbb{R}} S$, then $L_{A^{H}} \omega^{X, *}=0$. Since $\omega^{X *}$ is $J^{T_{\mathbb{R}}^{*} X}$-invariant, $L_{A^{H}} J^{T_{\mathbb{R}}^{*} X}$ lies in the symplectic Lie subalgebra of $\operatorname{End}\left(T_{\mathbb{R}}^{*} X\right)$. Equivalently $J^{T_{\mathbb{R}}^{*} X} L_{A^{H}} J^{T_{\mathbb{R}}^{*} X}$ is symmetric. Since $J^{T^{*} X, 2}=-1, L_{A^{H}} J^{T_{\mathbb{R}}^{*} X}$ anticommutes with $J^{T_{\mathbb{R}}^{*} X}$, so that $L_{A^{H}} J^{T_{\mathbb{R}}^{*} X}$ is also symmetric.

From (2.27), we find that if $B \in \overline{T S}, L_{\bar{B}^{H}} J^{T_{\mathbb{R}}^{*} X}$ vanishes on $T^{*} X$, and so it maps $\overline{T^{*} X}$ into $T^{*} X$. Using (2.25), we get (2.28). The proof of our theorem is completed.

Definition 2.6. Let $\nabla^{T_{\mathbb{R}}^{*} X}$ be the horizontal connection on $T_{\mathbb{R}}^{*} X$, such that if $A \in T_{\mathbb{R}} S$,

$$
\nabla_{A^{H}}^{T_{\mathbb{R}}^{*} X}=L_{A^{H}}+\frac{1}{2}\left(h^{T_{\mathbb{R}}^{*} X}\right)^{-1} L_{A^{H}} h^{T_{\mathbb{R}}^{*} X} .
$$




\section{HOLOMORPHIC AND DE RHAM TORSION}

Theorem 2.7. The horizontal connection $\nabla^{T_{\mathbb{R}}^{*} X}$ preserves the complex structure $J^{T_{\mathbb{R}}^{*} X}$ and the metric $h^{T_{\mathbb{R}}^{*} X}$. It induces on $T^{*} X$ the horizontal part of its holomorphic Hermitian connection.

Proof. Clearly $\nabla^{T_{\mathbb{R}}^{*} X}$ preserves $h^{T_{\mathbb{R}}^{*} X}$. By Theorem 2.5, $\left(h^{T_{\mathbb{R}}^{*} X}\right)^{-1} L_{A^{H}} h^{T_{\mathbb{R}}^{*} X}$ takes its values in the Lie algebra of the symplectic endomorphisms of $T_{\mathbb{R}}^{*} X$. Therefore $\nabla^{T_{\mathbb{R}}^{*} X}$ also preserves $\omega^{X *}$, and so it preserves the complex structure $J^{T_{\mathbb{R}}^{*} X}$. By Theorem 2.5, we find that when restricted to $T^{*} X$, $\nabla^{T_{\mathbb{R}}^{*} X^{\prime \prime}}$ coincides with the horizontal part of $\nabla^{T^{*} X^{\prime \prime}}$. Since $\nabla^{T_{\mathbb{R}}^{*} X}$ preserves the metric, we obtain the last part of our theorem.

\subsection{The holomorphic Hermitian connection on $T X$}

Definition 2.8. If $A \in T_{\mathbb{R}} S$, and if $B$ is a smooth section of $T_{\mathbb{R}} X$, set

$$
{ }^{0} \nabla_{A^{H}}^{T_{\mathbb{R}} X} B=\left[A^{H}, B\right] .
$$

Then ${ }^{0} \nabla_{A^{H}}^{T_{\mathbb{R}} X} B \in T_{\mathbb{R}} X$, and ${ }^{0} \nabla^{T_{\mathbb{R}} X}$ gives a horizontal connection on $T_{\mathbb{R}} X$. By Theorem 2.5, this horizontal connection preserves the symplectic form $\omega^{X}$.

Proposition 2.9. If $A \in T_{\mathbb{R}} S$, then ${ }^{0} \nabla_{A^{H}}^{T_{\mathbb{R}} X} J^{T_{\mathbb{R}} X}$ and $J^{T_{\mathbb{R}} X 0} \nabla_{A^{H}}^{T_{\mathbb{R}} X} J^{T_{\mathbb{R}} X}$ are symmetric tensors anticommuting with $J^{T_{\mathbb{R}}}$. Moreover

$$
\left(h^{T_{\mathbb{R}} X}\right)^{-10} \nabla_{A^{H}}^{T_{\mathbb{R}} X} h^{T_{\mathbb{R}} X}=-J^{T_{\mathbb{R}} X 0} \nabla_{A^{H}}^{T_{\mathbb{R}} X} J^{T_{\mathbb{R}} X} .
$$

Proof. Our proposition is an obvious consequence of Theorem 2.5.

DeFinition 2.10. Let $\nabla^{T_{\mathbb{R}} X}$ be the horizontal connection on $T_{\mathbb{R}} X$, then

$$
\nabla^{T_{\mathbb{R}} X}={ }^{0} \nabla^{T_{\mathbb{R}} X}+\frac{1}{2}\left(h^{T_{\mathbb{R}} X}\right)^{-10} \nabla^{T_{\mathbb{R}} X} h^{T_{\mathbb{R}} X} .
$$

TheOREM 2.11. The horizontal connection $\nabla^{T_{\mathbb{R}} X}$ preserves the complex structure $J^{T_{\mathbb{R}} X}$ and the metric $h^{T_{\mathbb{R}} X}$. It induces on $T X$ the horizontal part of its holomorphic Hermitian connection $\nabla^{T X}$. In particular ${ }^{0} \nabla^{T_{\mathbb{R}}} X^{\prime \prime} J^{T_{\mathbb{R}}}$ maps $T X$ into $\overline{T X}$ and vanishes on $\overline{T X}$.

Proof. Our theorem follows from Theorems 2.5 and 2.7 .

Remark 2.12. The fact that the horizontal connection $\nabla^{T_{\mathbb{R}}} X$ coincides with the horizontal part of $\nabla^{T X}$ was first established in [BGS88b, Theorem 1.5] and [B97, Theorems 1.1 and 2.3], using a different argument.

Definition 2.13. If $A \in T_{\mathbb{R}} S, B \in T_{\mathbb{R}} X$, set

$$
T(A, B)=\frac{1}{2}\left(h^{T_{\mathbb{R}} X}\right)^{-1}\left({ }^{0} \nabla_{A^{H}}^{T_{\mathbb{R}} X}\right) h^{T_{\mathbb{R}} X} B .
$$

Then by Theorem 2.11, the tensor $T$ is of type $(1,1)$. Also, if $A \in T S, B \in T X$, then $T(A, \bar{B}) \in T X$ and $T(\bar{A}, B) \in \overline{T X}$.

As in [BGS88b] and in [B97], we extend $T$ to an antisymmetric tensor of type $(1,1)$ on $M$ with values in $T_{\mathbb{R}} X$, which vanishes on pairs of elements of $T_{\mathbb{R}} X$, and which coincides with $T^{H}$ on pairs of horizontal vectors. In particular $T$ is a tensor of type $(1,1)$. Let $T^{(1,0)}, T^{(0,1)}$ be the components of $T$ in $T X, \overline{T X}$ respectively.

In the sequel, $\nabla^{T X}$ will denote the full holomorphic Hermitian connection on $T X$. It induces a corresponding connection on $T_{\mathbb{R}} X$, which we still denote $\nabla^{T_{\mathbb{R}}} X$.

Let $\nabla^{T_{\mathbb{R}} S}$ be a torsion free connection on $T_{\mathbb{R}} S$. Let $\nabla^{T_{\mathbb{R}} M}=\nabla^{T_{\mathbb{R}} S} \oplus \nabla^{T_{\mathbb{R}} X}$ be the obvious direct sum connection. Then by [BGS88b, $\S 1$ ] and [B97, Theorem 1.1], $T$ is exactly the torsion of the connection $\nabla^{T_{\mathbb{R}}} M$. 


\section{J.-M. Bismut}

\subsection{The Dolbeault operators on $M$}

The connection $\nabla^{T_{\mathbb{R}} X}$ induces a connection $\nabla^{\Lambda^{\bullet}\left(T_{\mathbb{R}}^{*} X\right)}$ on $\Lambda^{\bullet}\left(T_{\mathbb{R}}^{*} X\right)$, which is Hermitian and preserves the splitting

$$
\Lambda^{\bullet}\left(T_{\mathbb{R}}^{*} X\right) \otimes_{\mathbb{R}} \mathbb{C}=\bigoplus_{0 \leqslant p, q \leqslant \ell} \Lambda^{p}\left(T^{*} X\right) \widehat{\otimes} \Lambda^{q}\left(\overline{T^{*} X}\right)
$$

Definition 2.14. If $A \in T_{\mathbb{R}} S$, and if $s$ is a smooth section of $\Omega^{\bullet}(X, \mathbb{R})$, set

$$
\nabla_{A}^{\Omega^{\bullet}(X, \mathbb{R}), u} s=\nabla_{A^{H}}^{\Lambda^{\bullet}\left(T_{\mathbb{R}}^{*} X\right)} s .
$$

By [BGS88b, Theorem 1.14], $\nabla^{\Omega^{\bullet}(X, \mathbb{R}), u}$ is a Euclidean connection on $\Omega^{\bullet}(X, \mathbb{R})$, and its curvature is of type $(1,1)$. Moreover $\omega^{X}$ is parallel with respect to $\nabla^{\Omega^{\bullet}(X, \mathbb{R}), u}$. Note that $\nabla^{\Omega^{\bullet}(X, \mathbb{R}), u}$ extends to a Hermitian connection $\nabla^{\Omega^{*}(X, \mathbb{C}), u}$ on $\Omega^{\bullet}(X, \mathbb{C})$, which preserves the splitting $(2.3)$ of $\Omega^{\bullet}(X, \mathbb{C})$. Note that these results follow from Theorems 2.5 and 2.11 .

Let $w_{1}, \ldots, w_{\ell}$ be an orthonormal basis of $T X$, and let $f_{1}, \ldots, f_{m}$ be a basis of $T S$. We denote the corresponding conjugate or dual basis in the obvious way.

THEOREM 2.15. The following identity holds:

$$
d^{M}=d^{X}+\nabla^{\Omega^{\bullet}(X, \mathbb{R}), u}+i_{T}
$$

Moreover,

$$
\begin{aligned}
& \bar{\partial}^{M}=\bar{\partial}^{X}+\nabla^{\Omega^{\bullet}(X, \mathbb{R}), u^{\prime \prime}}+f^{\alpha} \bar{w}^{i} i_{T\left(f_{\alpha}, \bar{w}_{i}\right)}+f^{\alpha} \bar{f}^{\beta} i_{T^{(1,0)}\left(f_{\alpha}, \bar{f}_{\beta}\right)}, \\
& \partial^{M}=\partial^{X}+\nabla^{\Omega^{\bullet}(X, \mathbb{R}), u^{\prime}}+\bar{f}^{\alpha} w^{i} i_{T\left(\bar{f}_{\alpha}, w_{i}\right)}+f^{\alpha} \bar{f}^{\beta} i_{T^{(0,1)}\left(f_{\alpha}, \bar{f}_{\beta}\right)} .
\end{aligned}
$$

For any $p \in \mathbb{N}, \nabla^{\Omega^{\bullet}(X, \mathbb{R})^{\prime \prime}}$ maps $F^{p} \Omega^{\bullet}(X, \mathbb{R})$ into itself, and $\nabla^{\Omega^{\bullet}(X, \mathbb{R})^{\prime}}$ maps $F^{p} \Omega^{\bullet}(X, \mathbb{R})$ into $F^{p-1}$ $\Omega^{\bullet}(X, \mathbb{R})$. More precisely,

$$
\nabla^{\Omega^{\bullet}(X, \mathbb{R})}=\nabla^{\Omega^{\bullet}(X, \mathbb{R}), u}+f^{\alpha} \bar{w}^{i} i_{T\left(f_{\alpha}, \bar{w}_{i}\right)}+\bar{f}^{\alpha} w^{i} i_{T\left(\bar{f}_{\alpha}, w_{i}\right)} .
$$

Proof. We use the notation at the end of $\S 2.5$. Let $\nabla^{\Lambda^{\bullet}\left(T_{\mathbb{R}}^{*} M\right)}$ be the connection induced by $\nabla^{T_{\mathbb{R}} M}$ on $\Lambda^{\bullet}\left(T_{\mathbb{R}}^{*} M\right)$. Since $T$ is the torsion of $\nabla^{T_{\mathbb{R}} M}$,

$$
d^{M}=\nabla^{\Lambda^{\bullet}\left(T_{\mathbb{R}}^{*} M\right)}+i_{T},
$$

which is equivalent to (2.38).

Then we split the identity (2.38) by complex type, we use the facts that $\nabla^{\Omega^{\bullet}(X, \mathbb{R}), u}$ preserves the splitting (2.3), that $T$ is a tensor of type $(1,1)$, that $T\left(f_{\alpha}, \bar{w}_{i}\right) \in T X$ and that $T\left(\bar{f}_{\alpha}, w_{i}\right) \in \overline{T X}$, and we get (2.39). By comparing (2.22), (2.38) and (2.39), we obtain (2.40). The proof of our theorem is completed.

Remark 2.16. Needless to say, the results of Theorem 2.15 on $\nabla^{\Omega^{\bullet}(X, \mathbb{R})}$ imply classical results on variation of Hodge structures [GrT84]. Since $\bar{\partial}^{M, 2}=0,\left[\bar{\partial}^{M}, \partial^{M}\right]=0$, by (2.39), we get

$$
\begin{aligned}
& {\left[\nabla^{\Omega^{\bullet}(X, \mathbb{R}), u^{\prime \prime}}, \bar{\partial}^{X}\right]=0, \quad\left[\bar{\partial}^{X}, f^{\alpha} \bar{w}^{i} i_{T\left(f_{\alpha}, \bar{w}_{i}\right)}\right]=0, \quad \nabla^{\Omega^{\bullet}(X, \mathbb{R}), u^{\prime \prime}, 2}=0,} \\
& {\left[\nabla^{\Omega^{\bullet}(X, \mathbb{R}), u^{\prime \prime}}, f^{\alpha} \bar{w}^{i} i_{T\left(f_{\alpha}, \bar{w}_{i}\right)}\right]+\left[\bar{\partial}^{X}, f^{\alpha} \bar{f}^{\beta} i_{T^{(1,0)}\left(f_{\alpha}, \bar{f}_{\beta}\right)}\right]=0,} \\
& {\left[\nabla^{\Omega^{\bullet}(X, \mathbb{R}), u^{\prime \prime}}, f^{\alpha} \bar{f}^{\beta} i_{T^{(1,0)}\left(f_{\alpha}, \bar{f}_{\beta}\right)}\right]=0, \quad\left[\nabla^{\Omega^{\bullet}(X, \mathbb{R}), u^{\prime}}, \bar{\partial}^{X}\right]+\left[\partial^{X}, f^{\alpha} \bar{w}^{i} i_{T\left(f_{\alpha}, \bar{w}_{i}\right)}\right]=0,} \\
& {\left[\nabla^{\Omega^{\bullet}(X, \mathbb{R}), u^{\prime}}, f^{\alpha} \bar{w}^{i} i_{T\left(f_{\alpha}, \bar{w}_{i}\right)}\right]=0, \quad\left[\nabla^{\Omega^{\bullet}(X, \mathbb{R}), u^{\prime \prime}}, \bar{f}^{\alpha} w^{i} i_{T\left(\bar{f}_{\alpha}, w_{i}\right)}\right]=0,} \\
& {\left[\nabla^{\Omega^{\bullet}(X, \mathbb{R}), u^{\prime}}, \nabla^{\Omega^{\bullet}(X, \mathbb{R}), u^{\prime \prime}}\right]+\left[\bar{\partial}^{X}, f^{\alpha} \bar{f}^{\beta} i_{T^{(0,1)}\left(f_{\alpha}, \bar{f}_{\beta}\right)}\right]+\left[\partial^{X}, f^{\alpha} \bar{f}^{\beta} i_{T^{(1,0)}\left(f_{\alpha}, \bar{f}_{\beta}\right)}\right]=0 .}
\end{aligned}
$$

Note that, in (2.42), we have omitted most of the obvious conjugate equations. 


\section{Holomorphic AND DE RHAM TORSION}

Now we recall a result established in [BGS88b, Theorem 1.7].

Proposition 2.17. The following identities hold:

$$
\begin{gathered}
d^{X} \omega^{X}=0, \quad \nabla^{\Omega^{\bullet}(X, \mathbb{R}), u} \omega^{X}=0, \\
d^{X} \omega^{H}+i_{T} \omega^{X}=0, \quad \nabla^{\Omega^{\bullet}(X, \mathbb{R}), u} \omega^{H}=0 .
\end{gathered}
$$

Proof. We use (2.38) and the fact that $\omega^{M}$ is closed. Then we get easily the first and the last identities in (2.43), already obtained in (2.26). Since $\nabla^{T_{\mathbb{R}} X}$ preserves $J^{T_{\mathbb{R}} X}$ and the metric $h^{T X}$, it also preserves $\omega^{X}$, so that the second identity in (2.43) holds. The third identity now follows. Note that it breaks down into two identities, one of which is the third identity in (2.26).

\subsection{The Gauss-Manin connection}

Recall that the $\mathbb{Z}$-graded vector bundle $H^{\bullet}(X, \mathbb{R})$ is equipped with a flat connection $\nabla^{H^{\bullet}(X, \mathbb{R})}$ which preserves the $\mathbb{Z}$-grading. Indeed let $s$ be a smooth section of $\Omega^{p}(X, \mathbb{R})$ which is fibrewise closed. Extend $s$ to be a smooth section of $\Lambda^{p}\left(T_{\mathbb{R}}^{*} M\right)$. Since the form $d^{M} s$ restricts to a closed form along the fibres $X$, from the exact sequence

$$
0 \longrightarrow T_{\mathbb{R}}^{*} S \longrightarrow T_{\mathbb{R}}^{*} M \longrightarrow T_{\mathbb{R}}^{*} X \longrightarrow 0
$$

we find that $d^{M} s$ defines a smooth section of $T_{\mathbb{R}}^{*} S \widehat{\otimes} \Lambda^{p}\left(T_{\mathbb{R}}^{*} X\right)$. One verifies easily that this section is fibrewise closed and that its fibrewise $d^{X}$-cohomology class depends only on the fibrewise cohomology class of $s$. If $[s]$ denotes the associated section of $H^{\bullet}(X, \mathbb{R})$, we obtain the corresponding covariant derivative $\nabla^{H^{\bullet}(X, \mathbb{R})}[s]$. One verifies easily that $\nabla^{H^{\bullet}(X, \mathbb{R})}$ is a flat connection on $H^{\bullet}(X, \mathbb{R})$, which is compatible with Poincaré duality.

Let $\left[\omega^{X}\right] \in H^{2}(X, \mathbb{R})$ be the cohomology class of $\omega^{X}$. Since $\omega^{M}$ is closed, we find that

$$
\nabla^{H^{\bullet}(X, \mathbb{R})}\left[\omega^{X}\right]=0 .
$$

Using (2.22), we get a more explicit formula for $\nabla^{H^{\bullet}(X, \mathbb{R})}$. Indeed, if $s$ is taken as before, by (2.23), if $A \in T_{\mathbb{R}} S, \nabla_{A}^{\Omega^{*}(X, \mathbb{R})} s$ is $d^{X}$ closed. The corresponding representative in $H^{\bullet}(X, \mathbb{R})$ is just $\nabla^{H^{\bullet}(X, \mathbb{R})}[s]$. The third equation in $(2.23)$ guarantees that $\nabla^{H^{\bullet}(X, \mathbb{R})}$ is flat. Let $\nabla^{H^{\bullet}(X, \mathbb{C})}$ be the connection induced by $\nabla^{H^{\bullet}(X, \mathbb{R})}$ on $H^{\bullet}(X, \mathbb{C})$. Using Theorem 2.15, we recover the well-known fact $[\mathrm{GrT} 84]$ that $\nabla^{H^{\bullet}(X, \mathbb{C})^{\prime \prime}}$ maps $F^{p} H^{\bullet}(X, \mathbb{C})$ into itself, and that $\nabla^{H^{\bullet}(X, \mathbb{R})^{\prime}}$ maps $F^{p} H^{\bullet}(X, \mathbb{C})$ into $F^{p-1} H^{\cdot}(X, \mathbb{C})$.

Set

$$
D^{X}=d^{X}+d^{X, *}, \quad \mathcal{H}=\operatorname{ker} D^{X} .
$$

By Hodge theory, we have the canonical identification

$$
H^{\bullet}(X, \mathbb{R}) \simeq \mathcal{H}
$$

In the sequel, we identify $H^{\bullet}(X, \mathbb{R})$ and $\mathcal{H}$. Let $h^{H^{*}(X, \mathbb{R})}$ be the metric on $H^{\bullet}(X, \mathbb{R})$ which is induced by the scalar product $(2.5)$ on $\Omega^{\bullet}(X, \mathbb{R})$. Let $P^{\mathcal{H}}: \Omega^{\bullet}(X, \mathbb{R}) \rightarrow \mathcal{H}$ be the orthogonal projection operator with respect to $(2.5)$.

As observed by Ray and Singer [RS73], the metric $h^{H^{\bullet}(X, \mathbb{R})}$ depends only on the Kähler class $\left[\omega^{X}\right] \in H^{2}(X, \mathbb{R})$. This can be seen because using the Lefschetz decomposition of $H^{\bullet}(X, \mathbb{C})$, we can express the metric $h^{H^{\bullet}(X, \mathbb{R})}$ purely in cohomological terms, using the Hodge bilinear relations [GrHa78, p. 123].

Definition 2.18. Let $\nabla^{H^{\bullet}(X, \mathbb{R}), u}$ be the connection on $H^{\bullet}(X, \mathbb{R})$,

$$
\nabla^{H^{\bullet}(X, \mathbb{R}), u}=P^{\mathcal{H}} \nabla^{\Omega^{\bullet}(X, \mathbb{R}), u} .
$$




\section{J.-M. Bismut}

Now we have the result in [BLo95, Proposition 3.14].

Proposition 2.19. The connection $\nabla^{H^{\bullet}(X, \mathbb{R}), u}$ preserves $h^{H^{\bullet}(X, \mathbb{R})}$. Moreover

$$
\nabla^{H^{\bullet}(X, \mathbb{R}), u}=\nabla^{H^{\bullet}(X, \mathbb{R})}+\frac{1}{2}\left(h^{H^{\bullet}(X, \mathbb{R})}\right)^{-1} \nabla^{H^{\bullet}(X, \mathbb{R})} h^{H^{\bullet}(X, \mathbb{R})} .
$$

Remark 2.20. Equation (2.49) says that the metric connection $\nabla^{H^{*}(X, \mathbb{R}), u}$ is the canonical metric connection which is associated to the flat connection $\nabla^{H^{\bullet}(X, \mathbb{R})}$ and the metric $h^{H^{\bullet}(X, \mathbb{R})}$.

\subsection{The exterior algebra $\Lambda^{*}\left(T^{*} X\right)$ as a twisting vector bundle}

By Theorem 2.15, $\nabla^{\Omega^{\bullet}(X, \mathbb{C})^{\prime \prime}}$ maps $F^{p} \Omega^{\bullet}(X, \mathbb{C})$ into itself. So for any $p \in \mathbb{N}, \nabla^{\Omega^{\bullet}(X, \mathbb{C})^{\prime \prime}}$ induces an antiholomorphic connection $\nabla^{\mathrm{Gr} \Omega^{*} \Omega^{\bullet}(X, \mathbb{C})^{\prime \prime}}$ which preserves its bigrading. By $(2.40)$,

$$
\nabla^{\mathrm{Gr}} \Omega^{\bullet}(X, \mathbb{C})^{\prime \prime}=\nabla^{\Omega^{\bullet}(X, \mathbb{C}), u^{\prime \prime}} .
$$

More generally, in view of $(2.12)$, the projection of $\nabla^{\Omega^{*}(X, \mathbb{C})}$ on $\operatorname{Gr}^{\bullet} \Omega^{\bullet}(X, \mathbb{C})$ is well defined. By $(2.40)$, we find that this projection is just $\nabla^{\Omega^{\bullet}(X, \mathbb{C}), u}$, i.e. it is unitary.

In the sequel, we will consider $\Lambda^{\bullet}\left(T^{*} X\right)$ as a $\mathbb{Z}$-graded holomorphic vector bundle on $M$. In particular $\left(\Omega^{\bullet}\left(M, \Lambda^{\bullet}\left(T^{*} X\right)\right), \bar{\partial}^{M, \Lambda^{\bullet}\left(T^{*} X\right)}\right)$ denotes the Dolbeault complex of smooth sections of $\Lambda^{\bullet}\left(T^{*} M\right) \widehat{\otimes}$ $\Lambda^{\bullet}\left(T^{*} X\right)$ over $M$, equipped with the Dolbeault operator $\bar{\partial}^{M, \Lambda^{*}\left(T^{*} X\right)}$. Fibrewise, we may and we will still denote by $\bar{\partial}^{X}$ the corresponding operator acting on $\Omega^{\bullet}(X, \mathbb{C})$.

TheOREm 2.21. The following identity of operators holds:

$$
\bar{\partial}^{M, \Lambda^{\bullet}\left(T^{*} X\right)}=\bar{\partial}^{X}+\nabla^{\Omega^{\bullet}(X, \mathbb{C}), u^{\prime \prime}} .
$$

Proof. First we consider the case of the twisting bundle $\Lambda^{0}\left(T^{*} X\right) \simeq \mathbb{C}$. Then our theorem follows from the first identity in (2.39). Moreover by Theorem $2.7, \nabla^{T_{\mathbb{R}} X^{\prime \prime}}$ induces on $\Lambda^{\bullet}\left(T^{*} X\right)$ its antiholomorphic connection. By using (2.37), we get (2.51) in full generality.

Remark 2.22. Theorem 2.21 was established in [BGS88b, Theorem 2.8] in full generality in the case of a general twisting holomorphic vector bundle.

Note that $\operatorname{Gr} H^{\bullet}(X, \mathbb{C})=\bigoplus_{0 \leqslant p, q \leqslant \ell} H^{(p, q)}(X, \mathbb{C})$ is the direct image of $\Lambda^{\bullet}\left(T^{*} X\right)$. As explained in [BGS88c, $\S 3 \mathrm{c}$ ], the $H^{(p, q)}(X, \mathbb{C})$ can be equipped with a holomorphic structure $\nabla^{H^{(p, q)}(X, \mathbb{C})^{\prime \prime}}$. Its construction is formally the same as the construction of the Gauss-Manin connection $\nabla^{H^{*}(X, \mathbb{R})}$ in $\S 2.7$. By $(2.51)$, we find that $\nabla^{H^{(p, q)}(X, \mathbb{C})^{\prime \prime}}$ can be evaluated using $\nabla^{\Omega^{\bullet}(X, \mathbb{C}), u^{\prime \prime}}$. We denote by $\nabla^{\mathrm{Gr}^{\bullet}} H^{\bullet}(X, \mathbb{C})^{\prime \prime}$ the corresponding holomorphic structure on $\operatorname{Gr}^{\bullet} H^{\bullet}(X, \mathbb{C})$.

As we saw in the same section, $\nabla^{H^{\bullet}(X, \mathbb{C})^{\prime \prime}}$ maps $F^{p} H^{\bullet}(X, \mathbb{C})$ into itself. So it induces a holomorphic structure on $\operatorname{Gr} H^{\bullet}(X, \mathbb{C})$ which preserves the $H^{(p, q)}(X, \mathbb{C})$. The argument we gave at the beginning of the present subsection says that this holomorphic structure is exactly the one we just constructed.

Recall that $D^{X}$ and $\mathcal{H}$ were defined in (2.46). By (2.9),

$$
D^{X, 2}=2\left[\bar{\partial}^{X}, \bar{\partial}^{X, *}\right]=2\left[\partial^{X}, \partial^{X, *}\right] .
$$

By (2.52), we obtain the well-known splitting of $\mathcal{H}$,

$$
\mathcal{H} \otimes_{\mathbb{R}} \mathbb{C}=\bigoplus_{0 \leqslant p, q \leqslant \ell} \mathcal{H}^{(p, q)}(X, \mathbb{C})
$$

and the splitting in $(2.53)$ reflects the corresponding splitting $(2.13)$ of $H^{\bullet}(X, \mathbb{C})$.

Proposition 2.23. The connection $\nabla^{H^{*}(X, \mathbb{R}), u}$ preserves the $H^{(p, q)}(X, \mathbb{C})$. It coincides with the corresponding holomorphic Hermitian connection on $\operatorname{Gr} H^{\bullet}(X, \mathbb{C})$. 


\section{HOLOMORPHIC AND DE RHAM TORSION}

Proof. Since $\nabla^{\Omega^{\bullet}(X, \mathbb{R}), u}$ preserves the splitting $(2.3)$ of $\Omega^{\bullet}(X, \mathbb{R})$, the connection $\nabla^{H^{\bullet}(X, \mathbb{R}), u}$ also preserves the splitting $(2.13)$ of $H^{\bullet}(X, \mathbb{C})$. Moreover, as we saw before, its antiholomorphic part is just the canonical holomorphic structure on $\operatorname{Gr} H^{\bullet}(X, \mathbb{C})$. Therefore $\nabla^{H^{\bullet}(X, \mathbb{R}), u}$ induces the holomorphic Hermitian connection of $\mathrm{Gr} H^{\bullet}(X, \mathbb{C})$. The proof of our proposition is completed.

Remark 2.24. It is remarkable that the connection $\nabla^{H^{\bullet}(X, \mathbb{R}), u}$, which is the natural metric preserving connection on $H^{\bullet}(X, \mathbb{R})$ associated to the flat connection $\nabla^{H^{\bullet}(X, \mathbb{R})}$ and the metric $h^{H^{\bullet}(X, \mathbb{R})}$, induces the holomorphic metric connection on $\operatorname{Gr} H^{\bullet}(X, \mathbb{C})$.

This fact is made clearer if one realizes that the connection $\nabla^{H^{\bullet}(X, \mathbb{C}), u}$ is in fact metric independent. Indeed by Proposition 2.23, this is the case for its antiholomorphic part. Since the connection is real, this is the case also for its holomorphic part.

\section{The Levi-Civita superconnections}

In this section, we compare the complex and real versions of the Levi-Civita superconnection in [B86] as they appear in [BGS88b, BK92, BLo95]. More precisely, we give an explicit conjugation formula, in which the Lefschetz operators of the fibres $L, \Lambda$ appear explicitly.

This section is organized as follows. In $\S 3.1$, we consider the holomorphic Levi-Civita superconnection of [BGS88b, BK92, B97]. In $\S 3.2$, we construct the Levi-Civita superconnection in de Rham theory as in [BLo95]. In $\S 3.3$, we give the conjugation formula relating the two superconnections.

\subsection{The holomorphic Levi-Civita superconnection}

We make the same assumptions as in $\S \S 1$ and 2 , and we use the corresponding notation. In particular, we will use the notation of $\S 1.3$ with respect to the real tangent bundle $T_{\mathbb{R}} X$, equipped with the Hermitian product $h^{T X}$ and with the Kähler form $\omega^{X}$. If $U \in T_{\mathbb{R}} X$, the operators $c(U), \widehat{c}(U)$ act on $\Lambda^{\bullet}\left(T_{\mathbb{R}}^{*} X\right)$, and the operators $c_{T X}(U), c_{T X}(U)$ act on $\Lambda^{\bullet}\left(T_{\mathbb{R}}^{*} X\right) \otimes_{\mathbb{R}} \mathbb{C}$.

Definition 3.1. Set

$$
c_{\overline{T X}}\left(T^{H}\right)=f^{\alpha} \bar{f}^{\beta} c_{\overline{T X}}\left(T^{H}\left(f_{\alpha}, \bar{f}_{\beta}\right)\right) .
$$

Similarly, we also define $c \overline{T X}\left(T^{H(1,0)}\right)$ and $c_{\overline{T X}}\left(T^{H(0,1)}\right)$.

By (1.16),

$$
\frac{c \frac{c_{T X}}{\left(T^{H(1,0)}\right)}}{\sqrt{2}}=T^{H(1,0) *} \wedge, \quad \frac{c_{\overline{T X}}\left(T^{H(0,1)}\right)}{\sqrt{2}}=-i_{T^{H(0,1)}} .
$$

Also by [BGS88b, Theorem 2.6], or by Theorem 2.5, we get

$$
\frac{c_{\overline{T X}}\left(T^{H(1,0)}\right)}{\sqrt{2}}=-i\left[\bar{\partial}^{X}, \omega^{H}\right], \quad \frac{c_{\frac{T X}{T}}\left(T^{H(0,1)}\right)}{\sqrt{2}}=i\left[\bar{\partial}^{X, *}, \omega^{H}\right] .
$$

Now we follow [BGS88b, §2].

Definition 3.2. For $t>0$, set

$$
\begin{gathered}
B_{t}^{\prime \prime}=\sqrt{t} \bar{\partial}^{X}+\nabla^{\Omega^{\bullet}(X, \mathbb{C}), u^{\prime \prime}}-\frac{c_{\overline{T X}}\left(T^{H(1,0)}\right)}{2 \sqrt{2 t}}, \\
B_{t}^{\prime}=\sqrt{t} \bar{\partial}^{X, *}+\nabla^{\Omega^{\bullet}(X, \mathbb{C}), u^{\prime}}-\frac{c_{\overline{T X}}\left(T^{H(1,0)}\right)}{2 \sqrt{2 t}}, \\
B_{t}=\sqrt{t}\left(\bar{\partial}^{X}+\bar{\partial}^{X, *}\right)+\nabla^{\Omega^{\bullet}(X, \mathbb{C}), u}-\frac{c \overline{T X}\left(T^{H}\right)}{2 \sqrt{2 t}} .
\end{gathered}
$$




\section{J.-M. Bismut}

Then $B_{t}=B_{t}^{\prime \prime}+B_{t}^{\prime}$ is a superconnection on $\Omega^{\bullet}(X, \mathbb{C})$ in the sense of Quillen [Q85]. By [BGS88b, $\S 2], B_{u}$ is exactly the Levi-Civita superconnection of the fibration in the sense of [B86], which is associated to the metric $h^{T X} / t$, to the horizontal bundle $T_{\mathbb{R}}^{H} M$ and to the twisting vector bundle $\Lambda^{\bullet}\left(T^{*} X\right)$ equipped with the connection induced by $\nabla^{T X}$.

By (2.43) and (3.3), we recover the formula in [B97, (2.35)],

$$
\begin{aligned}
B_{t}^{\prime \prime} & =e^{-i \omega^{H} / 2 t} t^{N^{(0,1)} / 2}\left(\bar{\partial}^{X}+\nabla^{\Omega^{\bullet}(X, \mathbb{C}), u^{\prime \prime}}\right) t^{-N^{(0,1)} / 2} e^{i \omega^{H} / 2 t}, \\
B_{t}^{\prime} & =e^{i \omega^{H} / 2 t} t^{-N^{(0,1) / 2}}\left(\bar{\partial}^{X, *}+\nabla^{\Omega^{\bullet}(X, \mathbb{C}), u^{\prime}}\right) t^{N^{(0,1)} / 2} e^{-i \omega^{H} / 2 t} .
\end{aligned}
$$

Observe that by comparing (2.51) and the first identity in (3.5), we get

$$
B_{t}^{\prime \prime}=e^{-i \omega^{H} / 2 t} t^{N^{(0,1)} / 2} \bar{\partial}^{M, \Lambda^{\bullet}\left(T^{*} X\right)} t^{-N^{(0,1)} / 2} e^{i \omega^{H} / 2 t} .
$$

Set

$$
N_{t}^{(0,1)}=N^{(0,1)}+\frac{i \omega^{H}}{t} .
$$

By [BGS88b, Theorem 2.6], part of which follows from (3.6), we get

$$
\begin{gathered}
B_{t}^{\prime 2}=0, \quad B_{t}^{\prime \prime 2}=0, \quad B_{t}^{2}=\left[B_{t}^{\prime \prime}, B_{t}^{\prime}\right] \\
{\left[B_{t}^{\prime \prime}, B_{t}^{2}\right]=0, \quad\left[B_{t}^{\prime}, B_{t}^{2}\right]=0} \\
{\left[B_{t}^{\prime \prime}, N_{t}^{(0,1)}\right]=-2 t \frac{\partial}{\partial t} B_{t}^{\prime \prime}, \quad\left[B_{t}^{\prime}, N_{t}^{(0,1)}\right]=2 t \frac{\partial}{\partial t} B_{t}^{\prime} .}
\end{gathered}
$$

\subsection{The de Rham Levi-Civita superconnection}

Clearly, $N=N^{(1,0)}+N^{(0,1)}$ is the number operator of $\Lambda^{\bullet}\left(T_{\mathbb{R}}^{*} X\right)$, which induces the corresponding number operator on $\Omega^{\bullet}(X, \mathbb{R})$. Similarly, we define $\mathcal{N}$ as in (1.25).

Recall that the de Rham operator $d^{M}$ was evaluated in (2.22). Following [BLo95, $\S 3$, we will consider $d^{M}$ as a superconnection on $\Omega^{\bullet}(X, \mathbb{R})$.

Recall that $h^{\Omega^{\bullet}(X, \mathbb{R})}$ is the scalar product on $\Omega^{\bullet}(X, \mathbb{R})$ which was defined in $(2.5)$. Let $\nabla^{\Omega^{\bullet}(X, \mathbb{R}) *}$ be the adjoint superconnection to $\nabla^{\Omega^{\bullet}(X, \mathbb{R})}$. By definition,

$$
\nabla^{\Omega^{\bullet}(X, \mathbb{R}) *}=\nabla^{\Omega^{\bullet}(X, \mathbb{R})}+\left(h^{\Omega^{\bullet}(X, \mathbb{R})}\right)^{-1} \nabla^{\Omega^{\bullet}(X, \mathbb{R})} h^{\Omega^{\bullet}(X, \mathbb{R})} .
$$

Let $g_{1}, \ldots, g_{2 m}$ be a basis of $T_{\mathbb{R}} S$, and let $e_{1}, \ldots, e_{n}$ be an orthonormal basis of $T_{\mathbb{R}} X$. We denote the corresponding dual basis in the usual way. Using (2.21) and the fact that, by Theorem 2.5, the Kähler form $\omega^{X}$ is parallel with respect to $\nabla^{\Omega^{\bullet}(X, \mathbb{R})}$, we find easily that

$$
\nabla^{\Omega^{\bullet}(X, \mathbb{R}) *}=\nabla^{\Omega^{\bullet}(X, \mathbb{R})}-g^{\alpha} e^{i} i_{\left(h^{T} \mathbb{R}^{-1}\right.} L_{g_{\alpha}^{H}} h^{T_{\mathbb{R}} X} e_{i} .
$$

Proposition 3.3. The following identity holds:

$$
\nabla^{\Omega^{\bullet}(X, \mathbb{R}), u}=\frac{1}{2}\left(\nabla^{\Omega^{\bullet}(X, \mathbb{R})}+\nabla^{\Omega^{\bullet}(X, \mathbb{R}) *}\right) .
$$

Proof. This follows from (2.34), (2.37) and (3.10).

Definition 3.4. For $t>0$, set

$$
\begin{gathered}
C_{t}^{\dagger}=\sqrt{t} d^{X}+\nabla^{\Omega^{\bullet}(X, \mathbb{R})}+i_{T^{H}} / \sqrt{t}, \quad C_{t}^{\dagger *}=\sqrt{t} d^{X, *}+\nabla^{\Omega^{\bullet}(X, \mathbb{R}) *}-T^{H *} \wedge / \sqrt{t}, \\
C_{t}=\frac{1}{2}\left(C_{t}^{\dagger *}+C_{t}^{\dagger}\right), \quad D_{t}=\frac{1}{2}\left(C_{t}^{\dagger *}-C_{t}^{\dagger}\right) .
\end{gathered}
$$




\section{HOLOMORPHIC AND DE RHAM TORSION}

Then $C_{t}^{\dagger}, C_{t}^{\dagger *}, C_{t}$ are superconnections on $\Omega^{*}(X, \mathbb{R})$, and $D_{t}$ is an odd section of $\Lambda^{\bullet}\left(T_{\mathbb{R}}^{*} S\right) \widehat{\otimes}$ $\operatorname{End}\left(\Omega^{\bullet}(X, \mathbb{R})\right)$. Moreover

$$
C_{t}^{\dagger}=t^{N / 2} C_{1}^{\dagger} t^{-N / 2}, \quad C_{t}^{\dagger *}=t^{-N / 2} C_{1}^{\dagger *} t^{N / 2} .
$$

Comparing with (2.22), we can rewrite the first identity in (3.13) in the form

$$
C_{t}^{\dagger}=t^{N / 2} d^{M} t^{-N / 2}
$$

By [BLo95, Proposition 3.7], $C_{t}^{\dagger *}$ is the adjoint of the superconnection $C_{t}^{\prime}$. By [BLo95, Proposition $1.2]$, we get

$$
\begin{gathered}
C_{t}^{\dagger 2}=0, \quad C_{t}^{\dagger * 2}=0, \quad C_{t}^{2}=\frac{1}{4}\left[C_{t}^{\dagger *}, C_{t}^{\dagger}\right], \\
{\left[C_{t}^{\dagger}, C_{t}^{2}\right]=0, \quad\left[C_{t}^{\dagger *}, C_{t}^{2}\right]=0, \quad\left[C_{t}, D_{t}\right]=0, \quad D_{t}^{2}=-C_{t}^{2},} \\
{\left[C_{t}^{\dagger}, N\right]=-2 t \frac{\partial}{\partial t} C_{t}^{\dagger}, \quad\left[C_{t}^{\dagger *}, N\right]=2 t \frac{\partial}{\partial t} C_{t}^{\dagger *} .}
\end{gathered}
$$

Also, by (3.11) and (3.12), we have the formula

$$
C_{t}=\frac{\sqrt{t}}{2}\left(d^{X}+d^{X, *}\right)+\nabla^{\Omega^{\bullet}(X, \mathbb{R}), u}-\frac{c\left(T^{H}\right)}{2 \sqrt{t}} .
$$

Formula (3.16) for $C_{t}$ should be compared with formula (3.4) for $B_{t}$.

\subsection{A formula relating the Levi-Civita superconnections}

Recall that the fibres $X$ are equipped with the Kähler form $\omega^{X}$. Now we will use the notation of $\S 1$ with $V=T_{\mathbb{R}} X$. In particular the operators $L, \Lambda \in \operatorname{End}\left(\Omega^{\bullet}(X, \mathbb{R})\right)$ are defined as in (1.1) and (1.2). Similarly, the even operators $M, R$ are as in (1.20).

Theorem 3.5. For $t>0$, the following identities hold:

$$
\begin{aligned}
& e^{-\pi M / 4} i^{N^{(1,0)}} C_{2 t}^{\dagger} i^{-N^{(1,0)}} e^{\pi M / 4}=\sqrt{t}\left(\bar{\partial}^{X}+\bar{\partial}^{X, *}+i\left(\partial^{X}+\partial^{X, *}\right)\right)+\nabla^{\Omega^{\bullet}(X, \mathbb{C}), u} \\
& +i^{N^{(1,0)}}\left(\nabla^{\Omega^{\bullet}(X, \mathbb{C})}-\nabla^{\Omega^{\bullet}(X, \mathbb{C}), u}\right) i^{-N^{(1,0)}} \\
& -\frac{1}{2 \sqrt{2 t}}\left(c_{\overline{T X}}\left(T^{H}\right)-i c_{T X}\left(T^{H}\right)\right), \\
& e^{-\pi M / 4} i^{N^{(1,0)}} C_{2 t}^{\dagger *} i^{-N^{(1,0)}} e^{\pi M / 4}=\sqrt{t}\left(\bar{\partial}^{X}+\bar{\partial}^{X, *}-i\left(\partial^{X}+\partial^{X, *}\right)\right)+\nabla^{\Omega^{\bullet}(X, \mathbb{C}), u} \\
& -i^{N^{(1,0)}}\left(\nabla^{\Omega^{\bullet}(X, \mathbb{C})}-\nabla^{\Omega^{\bullet}(X, \mathbb{C}), u}\right) i^{-N^{(1,0)}} \\
& -\frac{1}{2 \sqrt{2 t}}\left(c_{\overline{T X}}\left(T^{H}\right)+i c_{T X}\left(T^{H}\right)\right) \text {. }
\end{aligned}
$$

In particular, for $t>0$,

$$
\begin{aligned}
e^{-\pi M / 4} i^{N^{(1,0)}} C_{2 t} i^{-N^{(1,0)}} e^{\pi M / 4}= & B_{t} \\
e^{-\pi M / 4} i^{N^{(1,0)}} D_{2 t} i^{-N^{(1,0)}} e^{\pi M / 4}= & i\left(-\sqrt{t}\left(\partial^{X}+\partial^{X, *}\right)+f^{\alpha} \bar{w}^{i} i_{T\left(f_{\alpha}, \bar{w}_{i}\right)}-\bar{f}^{\alpha} w^{i} i_{T\left(\bar{f}_{\alpha}, w_{i}\right)}\right. \\
& \left.-\frac{1}{2 \sqrt{2 t}} c_{T X}\left(T^{H}\right)\right) .
\end{aligned}
$$

For any $t>0$,

$$
\left[C_{t},-\frac{1}{2} \mathcal{N}+\frac{i}{2} M\right]=0
$$




\section{J.-M. Bismut}

Proof. Since $\nabla^{T X}$ is fibrewise torsion free, if $e_{1}, \ldots, e_{n}$ is an orthonormal basis of $T_{\mathbb{R}} X$, we get

$$
\begin{array}{cc}
d^{X}=\frac{1}{2}\left(c\left(e_{i}\right)+\widehat{c}\left(e_{i}\right)\right) \nabla_{e_{i}}^{\Lambda^{\bullet}\left(T_{\mathbb{R}}^{*} X\right)}, & d^{X, *}=\frac{1}{2}\left(c\left(e_{i}\right)-\widehat{c}\left(e_{i}\right)\right) \nabla_{e_{i}}^{\Lambda^{\bullet}\left(T_{\mathbb{R}}^{*} X\right)}, \\
\bar{\partial}^{X}+\bar{\partial}^{X, *}=\frac{1}{\sqrt{2}} c \frac{1}{T X}\left(e_{i}\right) \nabla_{e_{i}}^{\Lambda^{\bullet}\left(T_{\mathbb{R}}^{*} X\right)}, & \partial^{X}+\partial^{X, *}=\frac{1}{\sqrt{2}} c_{T X}\left(e_{i}\right) \nabla_{e_{i}}^{\Lambda^{\bullet}\left(T_{\mathbb{R}}^{*} X\right)} .
\end{array}
$$

Using Theorem 1.5 and the fact that the operator $M$ is parallel with respect to the connection $\nabla^{\Lambda^{\bullet}\left(T_{\mathbb{R}}^{*} X\right)}$ along the fibre $X$, we get

$$
\begin{aligned}
e^{-\pi M / 4} i^{N^{(1,0)}} d^{X} i^{-N^{(1,0)}} e^{\pi M / 4} & =\frac{1}{\sqrt{2}}\left(\bar{\partial}^{X}+\bar{\partial}^{X, *}+i\left(\partial^{X}+\partial^{X, *}\right)\right), \\
e^{-\pi M / 4} i^{N^{(1,0)}} d^{X, *} i^{-N^{(1,0)}} e^{\pi M / 4} & =\frac{1}{\sqrt{2}}\left(\bar{\partial}^{X}+\bar{\partial}^{X, *}-i\left(\partial^{X}+\partial^{X, *}\right)\right) .
\end{aligned}
$$

From (3.21), we get (3.17) in horizontal degree 0. Moreover using (3.11), we get

$$
\begin{aligned}
\nabla^{\Omega^{\bullet}(X, \mathbb{C})} & =\nabla^{\Omega^{\bullet}(X, \mathbb{C}), u}+\left(\nabla^{\Omega^{\bullet}(X, \mathbb{C})}-\nabla^{\Omega^{\bullet}(X, \mathbb{C}), u}\right), \\
\nabla^{\Omega^{\bullet}(X, \mathbb{C}) *} & =\nabla^{\Omega^{\bullet}(X, \mathbb{C}), u}-\left(\nabla^{\Omega^{\bullet}(X, \mathbb{C})}-\nabla^{\Omega^{\bullet}(X, \mathbb{C}), u}\right) .
\end{aligned}
$$

Also the connection $\nabla^{\Omega^{\bullet}(X, \mathbb{C}), u}$ preserves the bigrading of $\Omega^{\bullet}(X, \mathbb{C})$, and $L$ and $\Lambda$ are parallel with respect to $\nabla^{\Omega^{\bullet}(X, \mathbb{C}), u}$. By Theorem 2.5 , the operator $L$ is parallel with respect to $\nabla^{\Omega^{\bullet}(X, \mathbb{C})}$. Since $\Lambda$ is the adjoint of $L$ with respect to the symplectic form $\omega^{X}$, the operator $\Lambda$ is also parallel with respect to $\nabla^{\Omega^{\bullet}(X, \mathbb{C})}$. Using $(3.22)$, we get

$$
\begin{gathered}
e^{-\pi M / 4} i^{N^{(1,0)}} \nabla^{\Omega^{\bullet}(X, \mathbb{C})} i^{-N^{(1,0)}} e^{\pi M / 4}=\nabla^{\Omega^{\bullet}(X, \mathbb{C}), u}+i^{N^{(1,0)}}\left(\nabla^{\Omega^{\bullet}(X, \mathbb{C})}-\nabla^{\Omega^{\bullet}(X, \mathbb{C}), u}\right) i^{-N^{(1,0)},}, \\
e^{-\pi M / 4} i^{N^{(1,0)}} \nabla^{\Omega^{\bullet}(X, \mathbb{C}) *} i^{-N^{(1,0)}} e^{\pi M / 4}=\nabla^{\Omega^{\bullet}(X, \mathbb{C}), u}-i^{N^{(1,0)}}\left(\nabla^{\Omega^{\bullet}(X, \mathbb{C})}-\nabla^{\Omega^{\bullet}(X, \mathbb{C}), u}\right) i^{-N^{(1,0)}} .
\end{gathered}
$$

By (3.23), we get (3.17) in horizontal degree 1. Also, by Theorem 1.5,

$$
\begin{gathered}
e^{-\pi M / 4} i^{N^{(1,0)}} i_{T^{H}} i^{-N^{(1,0)}} e^{\pi M / 4}=\frac{1}{2}\left(i c_{T X}\left(T^{H}\right)-c_{\overline{T X}}\left(T^{H}\right)\right), \\
e^{-\pi M / 4} i^{N^{(1,0)}} T^{H *} \wedge i^{-N^{(1,0)}} e^{\pi M / 4}=\frac{1}{2}\left(i c_{T X}\left(T^{H}\right)+c_{\overline{T X}}\left(T^{H}\right)\right) .
\end{gathered}
$$

By (3.24), we get (3.17) in horizontal degree 2 .

By taking the half sum of the two equalities in (3.17), we get the first equality in (3.18). By taking the half difference, we obtain

$$
\begin{aligned}
& e^{-\pi M / 4} i^{N^{(1,0)}} D_{2 t} i^{-N^{(1,0)}} e^{\pi M / 4} \\
& \quad=-i \sqrt{t}\left(\partial^{X}+\partial^{X, *}\right)-i^{N^{(1,0)}}\left(\nabla^{\Omega^{\bullet}(X, \mathbb{C})}-\nabla^{\Omega^{\bullet}(X, \mathbb{C}), u}\right) i^{-N^{(1,0)}}-\frac{i}{2 \sqrt{2 t}} c_{T X}\left(T^{H}\right) .
\end{aligned}
$$

Also by (2.40) and (3.22), we get

$$
\begin{aligned}
\nabla^{\Omega^{\bullet}(X, \mathbb{C}), u}-\nabla^{\Omega^{\bullet}(X, \mathbb{C})} & =\frac{1}{2}\left(\nabla^{\Omega^{\bullet}(X, \mathbb{C}) *}-\nabla^{\Omega^{\bullet}(X, \mathbb{C})}\right) \\
& =-f^{\alpha} \bar{w}^{i} i_{T\left(f_{\alpha}, \bar{w}_{i}\right)}-\bar{f}^{\alpha} w^{i} i_{T\left(\bar{f}_{\alpha}, w_{i}\right)} .
\end{aligned}
$$

From (3.25) and (3.26), we obtain the second equality in (3.18).

We have the trivial

$$
\left[B_{t}, N^{(1,0)}\right]=0 \text {. }
$$

Using the fifth identity in (1.29) and the first identity in (3.18), we get (3.19). Needless to say, this last identity can be proved directly, by using (1.31). The proof of our theorem is completed.

Remark 3.6. Theorem 3.5 is remarkable. It indicates that the Dolbeault and de Rham superconnection formalisms are canonically conjugate. This fact will be very important in the sequel. 


\section{HOLOMORPHIC AND DE RHAM TORSION}

Observe that by (3.15), $C_{2 t}^{\dagger 2}=0, C_{2 t}^{\dagger * 2}=0$. So the squares of the right-hand sides of (3.17) also vanish. In horizontal degree 0 , this reflects the fact that

$$
\left(\bar{\partial}^{X}+\bar{\partial}^{X, *}\right)^{2}=\left(\partial^{X}+\partial^{X, *}\right)^{2}, \quad\left[\bar{\partial}^{X}+\bar{\partial}^{X, *}, \partial^{X}+\partial^{X, *}\right]=0,
$$

which also follows from (2.8) and (2.9). Similarly, by (3.15), $D_{2 t}^{2}=-C_{2 t}^{2}$, which implies a corresponding equality for the squares of the right-hand sides of (3.18). This equality is trivial in degree 0 .

\section{The vanishing of the equivariant holomorphic analytic torsion forms}

In this section, we prove that the equivariant holomorphic analytic torsion forms of a Kähler fibration vanish identically. This is done by using the formula relating the holomorphic Levi-Civita superconnection to the de Rham Levi-Civita superconnection, which was established in $\S 3$.

This section is organized as follows. In $\S 4.1$, we introduce the action of a compact Lie group $G$ on the total space $M$ of a Kähler fibration. In $\S 4.2$, we recall the construction of the superconnection forms of [BGS88b, BK92, M00]. In $\S 4.3$, we construct the corresponding analytic torsion forms. In $\S 4.4$, we recall the construction in [BLo95] of the de Rham superconnection forms. In $\S 4.5$, we compare the holomorphic and de Rham superconnection forms. In $\S 4.6$, we give an evaluation of the key form appearing in the definition of the analytic torsion. Finally, in $\S 4.7$, we show that the holomorphic torsion forms vanish identically.

\subsection{A group action}

We make the same assumptions as in $\S 3$, and we use the corresponding notation. Let $G$ be a compact Lie group acting holomorphically on $M$, preserving the fibres $X$ and the closed form $\omega^{M}$. In particular $G$ preserves the vector bundle $T^{H} M$ and the metric $T X$. Also $G$ acts on the complex $\left(\Omega^{\bullet}(X, \mathbb{R}), d^{X}\right)$ so that if $g \in G, s \in \Omega^{\bullet}(X, \mathbb{R})$,

$$
g s(x)=g_{*} s\left(g^{-1} x\right) .
$$

Also $G$ preserves the bigrading of $\Omega^{\bullet}(X, \mathbb{R})$. Since $G$ preserves the basic geometric data of the fibration, $G$ commutes with all the objects we considered in $\S 3$. If $g \in G$, set

$$
M_{g}=\{x \in M, g x=x\} .
$$

Then $M_{g}$ is a smooth complex submanifold of $M$, which fibres on $S$, with compact fibre $X_{g}$, the fixed point set of the fibres $X$. Moreover $\left.T^{H} M\right|_{M_{g}} \subset T M_{g}$ is a horizontal subbundle on $M_{g}$. Let $i_{g}: M_{g} \rightarrow M$ be the obvious embedding, and let $\pi_{g}: M_{g} \rightarrow S$ be the projection. Let $h^{T X_{g}}$ be the metric on $T X_{g}$ induced by $h^{T X}$. Then $\left(\pi,\left.T^{H} M\right|_{M_{g}}, h^{T X_{g}}\right)$ is a Kähler fibration, and $i^{*} \omega^{M}$ is an associated $(1,1)$ form.

If $g \in G$, let $L(g)$ be the Lefschetz number of $g$, i.e.

$$
L(g)=\operatorname{Tr}_{\mathrm{s}} H^{\bullet}(X, \mathbb{R})[g] .
$$

In the sequel we fix a square root $\sqrt{i}$ of $i$. Let $\Phi: \Lambda^{\bullet}\left(T_{\mathbb{R}}^{*} S\right) \otimes_{\mathbb{R}} \mathbb{C} \rightarrow \Lambda^{\bullet}\left(T_{\mathbb{R}}^{*} S\right) \otimes_{\mathbb{R}} \mathbb{C}$ be given by

$$
\Phi \alpha=(2 i \pi)^{-\operatorname{deg} \alpha / 2} \alpha .
$$

Let $P^{S}$ be the vector space of smooth complex forms on $S$ which are sums of forms of type $(p, p)$.

\subsection{The holomorphic superconnection forms}

Recall that

$$
\operatorname{Td}(x)=\frac{x}{1-e^{-x}}
$$




\section{J.-M. Bismut}

We identify Td with the corresponding multiplicative genus, the Todd genus. For $b \in \mathbb{R}$, set

$$
\operatorname{Td}^{b}(x)=\operatorname{Td}(x+b), \quad L^{b}(x)=1-e^{-b-x}, \quad L(x)=L^{0}(x) .
$$

We identify again $\mathrm{Td}^{b}, L^{b}, L$ with the corresponding multiplicative genera. Put

$$
\begin{aligned}
\operatorname{Td}^{\prime}\left(x_{1}, \ldots, x_{q}\right) & =\left.\frac{\partial}{\partial b} \operatorname{Td}^{b}\left(x_{1}, \ldots, x_{q}\right)\right|_{b=0}, \\
L^{\prime}\left(x_{1}, \ldots, x_{q}\right) & =\left.\frac{\partial}{\partial b} L^{b}\left(x_{1}, \ldots, x_{q}\right)\right|_{b=0} .
\end{aligned}
$$

Then (4.7) also defines corresponding genera.

Note that

$$
\sum_{0 \leqslant p \leqslant \ell}(-1)^{p} \operatorname{ch}\left(\Lambda^{p}\left(T^{*} X\right)\right)=L(T X), \quad \sum_{0 \leqslant p \leqslant \ell}(-1)^{p} p \operatorname{ch}\left(\Lambda^{p}\left(T^{*} X\right)\right)=-L^{\prime}(T X) .
$$

If $\theta \in \mathbb{R} \backslash 2 \pi \mathbb{Z}$, set

$$
\operatorname{Td}_{\theta}(x)=\frac{1}{1-e^{-x-i \theta}}, \quad \operatorname{Td}_{\theta}^{b}(x)=\frac{1}{1-e^{-x-b-i \theta}} .
$$

We identify $\operatorname{Td}_{\theta}(x), \operatorname{Td}_{\theta}^{b}(x)$ to the corresponding multiplicative genera.

In the sequel, we fix $g \in G$. Then $g$ acts as an automorphism of the normal bundle $N_{X_{g} / X}$ with locally constant eigenvalues $e^{i \theta}$. Let

$$
N_{X_{g} / X}=\bigoplus_{1 \leqslant j \leqslant q} N_{X_{g} / X}^{\theta_{j}}
$$

be the corresponding splitting of $N_{X_{g} / X}$ associated to the locally constant eigenvalues $e^{i \theta_{j}}$, with $0<\theta_{j}<2 \pi$. Set

$$
\operatorname{Td}_{g}(T X)=\operatorname{Td}\left(T X_{g}\right) \prod_{1 \leqslant j \leqslant q} \operatorname{Td}_{\theta_{j}}\left(N_{X_{g} / X}^{\theta_{j}}\right) .
$$

Then $\operatorname{Td}_{g}(T X)$ is exactly the characteristic class which appears in the Lefschetz formula of [ABo67, ABo68]. Similarly $\operatorname{Td}_{g}\left(T X, h^{T X}\right)$ denotes the closed form on $M_{g}$ which represents the cohomology class $\operatorname{Td}_{g}(T X)$ in Chern-Weil theory using the holomorphic Hermitian connection $\nabla^{T X}$. Similarly, we define the class $L_{g}(T X)$ and the corresponding form $L_{g}\left(T X, h^{T X}\right)$.

The class $\operatorname{Td}_{g}^{\prime}(T X)$ is defined as in (4.11), by taking the differential at $b=0$ of the class $\operatorname{Td}_{g}^{b}(T X)$ defined as in (4.11). In particular $\left(\mathrm{Td}_{g}^{\prime} / \mathrm{Td}_{g}\right)(T X)$ is a cohomology class which is obtained via the additive genera associated to $\operatorname{Td}^{\prime} / \mathrm{Td}(x)$ and to the $\operatorname{Td}_{\theta}^{\prime} / \operatorname{Td}_{\theta}(x)$. There are corresponding forms in Chern-Weil theory.

Finally note that the $H^{(p, q)}(X, \mathbb{C})$ are holomorphic $G$-bundles on $S$, which are equipped with the Hermitian metrics $h^{H^{(p, q)}(X, \mathbb{C})}$ which one obtains as in $(2.53)$, by identifying the corresponding cohomology classes to the associated harmonic forms. Let $\operatorname{ch}_{g}\left(H^{(p, q)}(X, \mathbb{C})\right)$ be the associated equivariant Chern character, which is an even cohomology class on $S$. The associated Chern forms $\operatorname{ch}_{g}\left(H^{(p, q)}(X, \mathbb{C}), h^{H^{(p, q)}(X, \mathbb{C})}\right)$ are evaluated using the obvious holomorphic Hermitian connection.

As explained in the Introduction, $\operatorname{Tr}_{\mathrm{s}}$ is our notation for the supertrace.

Definition 4.1. For $t>0$, set

$$
\begin{gathered}
\alpha_{t}=\Phi \operatorname{Tr}_{\mathrm{s}}\left[g \exp \left(-B_{t}^{2}\right)\right], \quad \beta_{t}=\frac{1}{\sqrt{2 i \pi}} \Phi \operatorname{Tr}_{\mathrm{s}}\left[g\left(\frac{\partial}{\partial t} B_{t}\right) \exp \left(-B_{t}^{2}\right)\right], \\
\gamma_{t}=\Phi \operatorname{Tr}_{\mathrm{s}}\left[g N_{t}^{(0,1)} \exp \left(-B_{t}^{2}\right)\right], \quad \delta_{t}=\Phi \operatorname{Tr}_{\mathrm{s}}\left[g N^{(1,0)} \exp \left(-B_{t}^{2}\right)\right] .
\end{gathered}
$$




\section{HOLOMORPHIC AND DE RHAM TORSION}

Theorem 4.2. For $t>0$, the forms $\alpha_{t}$ are closed, and their cohomology class coincides with the constant $L(g)$. The forms $\alpha_{t}, \gamma_{t}$ lie in $P^{S}$. Moreover there exist closed forms $C_{-1}, C_{0} \in P^{S}$ such that, as $t \rightarrow 0$,

$$
\alpha_{t}=L(g)+\mathcal{O}(t), \quad \gamma_{t}=\frac{C_{-1}}{t}+C_{0}+\mathcal{O}(t) .
$$

There exists a form $D_{0} \in P^{S}$ such that

$$
\begin{gathered}
C_{-1}=\int_{X_{g}} \frac{\omega^{M}}{2 \pi} c_{\max }\left(T X_{g}, h^{T X_{g}}\right), \\
C_{0}=\frac{n}{2} L(g)-\int_{X_{g}} \operatorname{Td}_{g}^{\prime}\left(T X, h^{T X}\right) L_{g}\left(T X, h^{T X}\right)+\frac{\bar{\partial} \partial}{2 i \pi} D_{0} .
\end{gathered}
$$

As $t \rightarrow+\infty$,

$$
\begin{gathered}
\alpha_{t}=L(g)+\mathcal{O}(1 / \sqrt{t}) \\
\gamma_{t}=\sum_{0 \leqslant p, q \leqslant \ell}(-1)^{p+q} q \operatorname{ch}_{g}\left(H^{(p, q)}(X, \mathbb{C}), h^{H^{(p, q)}(X, \mathbb{C})}\right)+\mathcal{O}(1 / \sqrt{t}) .
\end{gathered}
$$

Moreover

In particular

$$
\frac{\partial}{\partial t} \alpha_{t}=-d \beta_{t}, \quad \beta_{t}=\frac{1}{4 i \pi}(\partial-\bar{\partial}) \frac{\gamma_{t}}{t}
$$

$$
\frac{\partial}{\partial t} \alpha_{t}=-\frac{\bar{\partial} \partial}{2 i \pi} \frac{\gamma_{t}}{t}
$$

The form $\delta_{t}$ lies in $P^{S}$, it is closed and its cohomology class $\left[\delta_{t}\right]$ is given by

$$
\left[\delta_{t}\right]=\sum_{0 \leqslant p, q \leqslant \ell}(-1)^{p+q} p \operatorname{ch}_{g}\left(H^{(p, q)}(X, \mathbb{C})\right) .
$$

As $t \rightarrow 0$,

$$
\delta_{t}=-\int_{X_{g}} \operatorname{Td}_{g}\left(T X, h^{T X}\right) L_{g}^{\prime}\left(T X, h^{T X}\right)+\mathcal{O}(t),
$$

and as $t \rightarrow+\infty$,

$$
\delta_{t}=\sum_{0 \leqslant p, q \leqslant \ell}(-1)^{p+q} p \operatorname{ch}_{g}\left(H^{(p, q)}(X, \mathbb{C}), h^{H^{(p, q)}(X, \mathbb{C})}\right)+\mathcal{O}(1 / \sqrt{t}) .
$$

The forms $\Phi^{-1} \alpha_{t}$ are real, and the forms $\Phi^{-1}\left(\gamma_{t}-(n / 4) \alpha_{t}\right)$ are purely imaginary. Finally, if $g=1$, the forms $\alpha_{t}, \beta_{t}, \gamma_{t}, \delta_{t}$ are real.

Proof. The fact that the forms $\alpha_{t}, \delta_{t}$ are closed and that their cohomology class does not depend on $t$ was established in [B86, Theorem 3.4] and by [M00, Theorem 2.10] in the equivariant case. By [BGS88b, Theorems 2.2 and 2.9], the forms $\alpha_{t}, \gamma_{t}$ and $\delta_{t}$ lie in $P^{S}$. By [B86, Theorems 4.12 and 4.16] and by [M00, Theorem 2.10] in the equivariant case, and by the first identity in (4.8), as $t \rightarrow 0$,

$$
\alpha_{t}=\int_{X_{g}} c_{\max }\left(T X_{g}, \nabla^{T X_{g}}\right)+\mathcal{O}(t)
$$

Using the standard Lefschetz formula, we find that (4.21) is just the first identity in (4.13). The same references, combined with the second identity in (4.8), show that (4.19) holds. By [BGS88b, Theorem 2.16] and [M00, Theorem 2.6], we get the second identity in (4.13) and also the identities in (4.14). 
By [BeGeV92, Theorem 9.19], we know that, as $t \rightarrow+\infty$,

$$
\begin{gathered}
\alpha_{t}=\operatorname{ch}_{g}\left(H^{\bullet}(X, \mathbb{R}), \nabla^{H^{\bullet}(X, \mathbb{R}), u}\right)+\mathcal{O}(1 / \sqrt{t}), \\
\gamma_{t}=\Phi \operatorname{Tr}_{\mathrm{s}}^{H^{\bullet}(X, \mathbb{R})}\left[g N^{(0,1)} \exp \left(-\nabla^{H^{\bullet}(X, \mathbb{R}) u, 2}\right)\right]+\mathcal{O}(1 / \sqrt{t}) .
\end{gathered}
$$

By Propositions 2.19 and 2.23, the holomorphic Hermitian connection $\nabla^{H^{\bullet}(X, \mathbb{R}), u}$ is the Euclidean connection on $H^{\bullet}(X, \mathbb{R})$ which is canonically associated to the flat connection $\nabla^{H^{*}(X, \mathbb{R})}$ and the metric $h^{H^{\bullet}(X, \mathbb{R})}$. By [BLo95, Proposition 1.3], we get

$$
\operatorname{ch}_{g}\left(H^{\bullet}(X, \mathbb{R}), \nabla^{H^{\bullet}(X, \mathbb{R}), u}\right)=L(g) .
$$

By (4.22) and (4.23), we get (4.15). The same argument shows that (4.20) also holds.

Equations (4.16) and (4.17) were established in [BGS88b, Theorem 2.9]. Note that (4.17) follows from (4.16).

Let $\mu: \Omega^{\bullet}(X, \mathbb{C}) \rightarrow \Omega^{\bullet}(X, \mathbb{C})$ be such that, if $s \in \Omega^{i}(X, \mathbb{C})$, then

$$
\mu s=(-1)^{i(i+1) / 2} \overline{* s} \text {. }
$$

Then $\mu$ is an even operator, such that

$$
\mu^{2}=(-1)^{n(n+1) / 2}
$$

Using (3.4), one verifies easily that

$$
\mu^{-1} B_{t} \mu=B_{t}
$$

Moreover,

$$
\mu^{-1} N_{t}^{(0,1)} \mu=\frac{n}{2}-N_{t}^{(0,1)} .
$$

Finally the operator $\mu$ commutes with $g$. By (4.26), we see that the forms $\Phi^{-1} \alpha_{t}$ are real. Similarly, since $\mu$ is even, using the fact that supertraces vanish on supercommutators and also (4.26) and (4.27), we get

$$
\operatorname{Re} \Phi^{-1} \gamma_{t}=\frac{n}{4} \Phi^{-1} \alpha_{t}
$$

Finally the fact that when $g=1$, the forms in (4.12) are real follows from [BF86, Theorem 1.5]. The proof of our theorem is completed.

\subsection{The equivariant holomorphic analytic torsion forms}

Now we recall the definition by [BGS88b, Definition 2.19] and [BK92, Definition 3.8] of the analytic torsion forms, and by [M00, Definition 2.11] for the equivariant case.

Definition 4.3. For $s \in \mathbb{C}, \operatorname{Re}(s)>1$, set

$$
\zeta^{1}(s)=-\frac{1}{\Gamma(s)} \int_{0}^{1} t^{s-1}\left(\gamma_{t}-\gamma_{+\infty}\right) d t
$$

For $s \in \mathbb{C}, \operatorname{Re}(s)<1 / 2$, set

$$
\zeta^{2}(s)=-\frac{1}{\Gamma(s)} \int_{1}^{+\infty} t^{s-1}\left(\gamma_{t}-\gamma_{+\infty}\right) d t
$$

By Theorem 4.2, $\zeta^{1}(s), \zeta^{2}(s)$ extend to functions of $s$ which are holomorphic near $s=0$.

Definition 4.4. Set

$$
T_{g}\left(\omega^{M}\right)=\frac{\partial}{\partial s}\left(\zeta^{1}+\zeta^{2}\right)(0)
$$

Then $T_{g}\left(\omega^{M}\right)$ is a smooth form on $S$, which lies in $P^{S}$. The form $T_{g}\left(\omega^{M}\right)$ is called an equivariant holomorphic analytic torsion form. 
Now we recall a result of [BGS88b, Theorem 2.20], [BK92, Theorem 3.9] and [M00, Theorem $2.12]$.

Proposition 4.5. The form $T_{g}\left(\omega^{M}\right)$ is such that

$$
\frac{\bar{\partial} \partial}{2 i \pi} T_{g}\left(\omega^{M}\right)=0
$$

Proof. Our result follows from Theorem 4.2, including the fact that $\alpha_{0}=\alpha_{+\infty}$.

Clearly, we can as well construct forms $\tau_{g}^{ \pm}\left(\omega^{M}\right) \in P^{S}$, by replacing $\gamma_{t}$ by $\gamma_{t} \pm \delta_{t}$ in (4.29) and (4.30). Since $\delta_{t}$ is closed, and its cohomology class is constant, it is clear that the $\tau_{g}^{ \pm}\left(\omega^{M}\right)$ are also $\bar{\partial} \partial$-closed forms, which lie in the same $P^{S} / P^{S, 0}$ class as $T_{g}\left(\omega^{M}\right)$.

\subsection{The de Rham superconnection forms}

Put

$$
h(x)=x \exp \left(x^{2}\right)
$$

Definition 4.6. For $t>0$, set

$$
\begin{gathered}
a_{t}=\Phi \operatorname{Tr}_{\mathrm{s}}\left[g \exp \left(-C_{t}^{2}\right)\right], \quad b_{t}=\frac{1}{\sqrt{2 i \pi}} \Phi \operatorname{Tr}_{\mathrm{s}}\left[g\left(\frac{\partial}{\partial t} C_{t}\right) \exp \left(-C_{t}^{2}\right)\right], \\
c_{t}=\sqrt{2 i \pi} \Phi \operatorname{Tr}_{\mathrm{s}}\left[g h\left(D_{t}\right)\right], \quad d_{t}=\Phi \operatorname{Tr}_{\mathrm{s}}\left[g N \exp \left(-C_{t}^{2}\right)\right] .
\end{gathered}
$$

Now we have the results in [BLo95, Theorems 2.27 and 3.15].

Proposition 4.7. The following identities hold:

$$
a_{t}=L(g), \quad b_{t}=0, \quad c_{t}=0, \quad d_{t}=\frac{n}{2} L(g) .
$$

Proof. The first identity was established in [BLo95, Theorem 3.15] for $g=1$, the proof for the general case being exactly the same. The second identity can be derived from the first one. We give here a direct proof. By the last two identities in (3.15),

$$
2 t\left(\frac{\partial}{\partial t} C_{t}\right)=\left[D_{t}, N\right]
$$

Also by $(3.15), D_{t}^{2}=-C_{t}^{2}$, so that

$$
b_{t}=\frac{1}{\sqrt{2 i \pi} 2 t} \Phi \operatorname{Tr}_{\mathrm{s}}\left[\left[D_{t}, g N \exp \left(-C_{t}^{2}\right)\right]\right]=0 .
$$

Since the fibres $X$ are orientable and $G$ preserves the orientation, the same argument as in [BLo95, Theorems 2.27 and 3.26] establishes the last two identities in (4.35).

\subsection{Comparing the holomorphic and de Rham superconnection forms}

Now we will use our results in $\S 3$ and in the above subsections to compare the superconnection forms in the holomorphic and de Rham cases.

TheOrem 4.8. For any $t>0$, the following identities hold:

$$
\alpha_{t}=L(g), \quad \beta_{t}=0, \quad d \gamma_{t}=0 .
$$


Moreover

$$
\begin{gathered}
\gamma_{t}=\Phi \operatorname{Tr}_{\mathrm{S}}\left[g\left(\frac{1}{2} \mathcal{N}+\frac{i}{2} M+\frac{i \omega^{H}}{t}\right) \exp \left(-C_{2 t}^{2}\right)\right]+\frac{n}{4} L(g), \\
\delta_{t}=\Phi \operatorname{Tr}_{\mathrm{S}}\left[g\left(-\frac{1}{2} \mathcal{N}+\frac{i}{2} M\right) \exp \left(-C_{2 t}^{2}\right)\right]+\frac{n}{4} L(g) .
\end{gathered}
$$

In particular

$$
\begin{aligned}
\gamma_{t}+\delta_{t} & =\Phi \operatorname{Tr}_{\mathrm{s}}\left[g i\left(M+\frac{\omega^{H}}{t}\right) \exp \left(-C_{2 t}^{2}\right)\right]+\frac{n}{2} L(g) \\
& =\Phi \operatorname{Tr}_{\mathrm{s}}\left[g i\left(2 L+\frac{\omega^{H}}{t}\right) \exp \left(-C_{2 t}^{2}\right)\right]+\frac{n}{2} L(g), \\
\gamma_{t}-\delta_{t} & =\Phi\left[g\left(\mathcal{N}+\frac{i \omega^{H}}{t}\right) \exp \left(-C_{2 t}^{2}\right)\right]
\end{aligned}
$$

Moreover the forms $\Phi^{-1} \gamma_{t}-(n / 4) L(g)$ and $\Phi^{-1} \delta_{t}-(n / 4) L(g)$ are purely imaginary.

The forms $T_{g}\left(\omega^{M}\right)$ and $\tau_{g}^{ \pm}\left(\omega^{M}\right)$ lie in $P^{S}$, they are closed, and their images by $\Phi^{-1}$ are purely imaginary.

Proof. We use the identity (3.18) in Theorem 3.5. Since supertraces vanish on supercommutators, we get

$$
\alpha_{t}=a_{2 t}, \quad \beta_{t}=2 b_{2 t} .
$$

Comparing with Proposition 4.7, we obtain the first two identities in (4.38). Also using the second identity in (4.16) and the fact that $\gamma_{t} \in P^{S}$, we get the last identity in (4.38).

Using Theorem 1.5 and (3.18), we get

$$
\gamma_{t}=\Phi \operatorname{Tr}_{\mathrm{s}}\left[g\left(\frac{1}{2} \mathcal{N}+\frac{n}{4}+\frac{i}{2} M+\frac{i \omega^{H}}{t}\right) \exp \left(-C_{2 t}^{2}\right)\right] .
$$

By (4.35) and (4.42), we get the first identity in (4.39). The proof of the second identity is similar.

Let $\nu: \Omega^{\bullet}(X, \mathbb{R}) \rightarrow \Omega^{\bullet}(X, \mathbb{R})$ be such that, if $s \in \Omega^{i}(X)$,

$$
\nu s=(-1)^{i(i+1) / 2} * s .
$$

Then

$$
\nu^{2}=(-1)^{n(n+1) / 2} .
$$

By [BLo95, Equation (2.106) and Lemma 2.36],

$$
C_{t}=\nu^{-1} C_{t} \nu
$$

Moreover, we have the trivial

$$
\nu^{-1} \Lambda \nu=-L
$$

Since $\nu$ is an even operator, using (4.45) and (4.46), we get

$$
\operatorname{Tr}_{\mathrm{s}}\left[g \Lambda \exp \left(-C_{t}^{2}\right)\right]=-\operatorname{Tr}_{\mathrm{s}}\left[g L \exp \left(-C_{t}^{2}\right)\right]
$$

The remaining identities in (4.40) follow from (4.47) and from the previous arguments.

Since the operators $N^{(1,0)}$ and $N^{(0,1)}$ are conjugate and the operators $g$ and $C_{2 t}^{2}$ are real, we find that

$$
\operatorname{Tr}_{\mathrm{s}}\left[g \mathcal{N} \exp \left(-C_{2 t}^{2}\right)\right]
$$

is purely imaginary. By (4.39), we get the stated results on the forms $\gamma_{t}$ and $\delta_{t}$. 


\section{HolOMORPHIC AND DE RHAM TORSION}

By (4.29), (4.30) and the third identity in (4.38), we find that the form $T_{g}\left(\omega^{M}\right)$ is closed. The fact that the image of the torsion forms by $\Phi^{-1}$ is purely imaginary follows from the above results. The proof of our theorem is completed.

Remark 4.9. We have reobtained some of the results of Theorem 4.2 in a stronger form.

\subsection{An evaluation of $\left[\gamma_{t}\right]$}

Set

$$
A^{\dagger}=C_{1}^{\dagger}, \quad A_{t}^{\dagger *}=t^{-N} C_{1}^{\dagger *} t^{N}, \quad A_{t}=\frac{1}{2}\left(A_{t}^{\dagger *}+A^{\dagger}\right) .
$$

Then when replacing $h^{T X}$ by $h^{T X} / t, A_{t}$ is just the corresponding $C_{1}$. By (3.13) and (4.48),

$$
A_{t}=t^{-N / 2} C_{t} t^{N / 2} \text {. }
$$

Definition 4.10. Put

$$
\epsilon_{t}=\Phi \operatorname{Tr}_{\mathrm{s}}\left[g i \omega^{M} \exp \left(-A_{t}^{2}\right)\right]
$$

Theorem 4.11. The forms $\epsilon_{t}$ are closed, and their cohomology class does not depend on $t>0$. Moreover

$$
\gamma_{t}+\delta_{t}=\frac{\epsilon_{2 t}}{t}+\frac{n}{2} L(g)
$$

Proof. By (3.14) and (4.48), $A^{\dagger}=d^{M}$, so that, since $\omega^{M}$ is closed,

$$
\left[A^{\dagger}, \omega^{M}\right]=0 .
$$

Moreover, by (3.15) and (4.48),

$$
\left[A^{\dagger}, A_{t}^{2}\right]=0 .
$$

Using (4.52) and (4.53) and the fact that supertraces vanish on supercommutators, we get

$$
d \Phi^{-1} \epsilon_{t}=\operatorname{Tr}_{\mathrm{s}}\left[\left[A^{\dagger}, g i \omega^{M} \exp \left(-A_{t}^{2}\right)\right]\right]=0 .
$$

Moreover, by (3.15) and (4.48),

$$
A_{t}^{2}=\frac{1}{4}\left[A^{\dagger}, A_{t}^{\dagger *}\right]
$$

so that

$$
\frac{\partial}{\partial t} \Phi^{-1} \epsilon_{t}=\left.\frac{\partial}{\partial b} \operatorname{Tr}_{\mathrm{s}}\left[g i \omega^{M} \exp \left(-A_{t}^{2}-\frac{b}{4}\left[A^{\dagger}, \frac{\partial}{\partial t} A_{t}^{\dagger *}\right]\right)\right]\right|_{b=0} .
$$

By (4.52) and (4.53), we can rewrite (4.56) in the form

$$
\frac{\partial}{\partial t} \Phi^{-1} \epsilon_{t}=\left.d \frac{\partial}{\partial b} \operatorname{Tr}_{\mathrm{s}}\left[g i \omega^{M} \exp \left(-A_{t}^{2}-\frac{b}{4} \frac{\partial}{\partial t} A_{t}^{\dagger *}\right)\right]\right|_{b=0} .
$$

So we have proved that $\epsilon_{t}$ is closed and that its cohomology class does not depend on $t>0$.

Clearly

$$
t^{N / 2} \omega^{M} t^{-N / 2}=\left(t L+\omega^{H}\right)
$$

By (4.49) and (4.58), we get

$$
\epsilon_{t}=\Phi \operatorname{Tr}_{\mathrm{s}}\left[g i\left(t L+\omega^{H}\right) \exp \left(-C_{t}^{2}\right)\right]
$$

By (4.40) and (4.59), we get (4.51). The proof of our theorem is completed. 


\section{J.-M. Bismut}

Remark 4.12. The critical fact in our proof that $\epsilon_{t}$ is closed is given by (4.52), which is equivalent to

$$
\left[C_{2 t}^{\dagger}, 2 t L+\omega^{H}\right]=0 .
$$

Using the last identity in (1.29) and the first identity in (3.17), we see that (4.60) is equivalent to

$$
\begin{aligned}
& {\left[\sqrt{t}\left(\bar{\partial}^{X}+\bar{\partial}^{X, *}+i\left(\partial^{X}+\partial^{X, *}\right)\right)+\nabla^{\Omega^{\bullet}(X, \mathbb{C}), u}+i^{N^{(1,0)}}\left(\nabla^{\Omega^{\bullet}(X, \mathbb{R})}-\nabla^{\Omega^{\bullet}(X, \mathbb{C}), u}\right) i^{-N^{(1,0)}}\right.} \\
& \left.-\frac{1}{2 \sqrt{2 t}}\left(c_{T X}\left(T^{H}\right)-i c_{T X}\left(T^{H}\right)\right), N-\frac{n}{2}-M+\frac{i \omega^{H}}{t}\right]=0 .
\end{aligned}
$$

One must admit that (4.61) is not entirely trivial.

The above demonstrates that, although the holomorphic and de Rham formalisms are ultimately equivalent, identities which are easy to see in one formalism are not necessarily trivial in the other.

Let $\left[\epsilon_{t}\right] \in H^{\bullet}(S, \mathbb{C})$ be the cohomology class of $\epsilon_{t}$.

Theorem 4.13. For any $t>0$,

$$
\left[\epsilon_{t}\right]=\int_{X_{g}} \frac{\omega^{M}}{2 \pi} c_{\max }\left(T X_{g}\right)
$$

Moreover, for any $t>0$,

$$
\left[\gamma_{t}\right]=\int_{X_{g}} \frac{\omega^{M}}{2 \pi} c_{\max }\left(T X_{g}\right) \frac{1}{t}+\frac{n}{2} L(g)-\int_{X_{g}} \operatorname{Td}_{g}^{\prime}(T X) L_{g}(T X) .
$$

Proof. By Theorem 4.11, for any $t>0$,

$$
\left[\gamma_{t}\right]+\left[\delta_{t}\right]=\frac{\left[\epsilon_{2 t}\right]}{t}+\frac{n}{2} L(g)
$$

By Theorems 4.2 and 4.11, $\left[\delta_{t}\right]$ and $\left[\epsilon_{t}\right]$ do not depend on $t>0$. By using (4.13) and (4.14) in Theorem 4.2 together with (4.64), we obtain (4.62). By (4.64), [t $\left.\gamma_{t}\right]$ is an affine function of $t>0$. Comparing with (4.13) and (4.14) again, we get (4.63). The proof of our theorem is completed.

Remark 4.14. It is interesting to reobtain (4.63) in another way. In fact, by (4.19), (4.62) and (4.64), we get

$$
\left[\gamma_{t}\right]=\int_{X_{g}} \frac{\omega^{M}}{2 \pi} c_{\max }\left(T X_{g}\right) \frac{1}{t}+\frac{n}{2} L(g)+\int_{X_{g}} \operatorname{Td}_{g}(T X) L_{g}^{\prime}(T X) .
$$

We will show that (4.65) coincides with (4.63). Indeed

$$
\operatorname{Td}^{b}(x) L^{b}(x)=x+b .
$$

By degree theoretic considerations, we get

$$
\int_{X_{g}} \operatorname{Td}_{g}^{b}(T X) L_{g}^{b}(T X)=\int_{X_{g}} c_{\max }\left(T X_{g}\right) .
$$

By differentiating (4.67) in $b$ at $b=0$, we find that (4.63) and (4.65) are equivalent.

\subsection{The vanishing of the analytic torsion forms}

Recall that by Theorem 4.8, the form $T_{g}\left(\omega^{M}\right)$ lies in $P^{S}$ and is closed.

TheOREM 4.15. The following identity holds:

$$
T_{g}\left(\omega^{M}\right)=0 \quad \text { in } H^{\text {even }}(S, \mathbb{C}) .
$$




\section{HolOMORPHIC AND DE RHAM TORSION}

Proof. We can define the integral $\int_{0}^{+\infty} t^{s-1} d t$ for $s \in \mathbb{C}^{*}$ by splitting it as

$$
\int_{0}^{+\infty} t^{s-1} d t=\int_{0}^{a} t^{s-1} d t+\int_{a}^{+\infty} t^{s-1} d t
$$

by defining each of the terms of the sum on its natural domain of definition, and extending the corresponding expression meromorphically. With this convention, we get

$$
\int_{0}^{+\infty} t^{s-1} d t=0
$$

Now we used precisely this convention to define the form $T_{g}\left(\omega^{M}\right)$ in Definition 4.4. Moreover by (4.63), $t\left[\gamma_{t}\right]$ is an affine function of $t>0$. From (4.70), we get (4.68). The proof of our theorem is completed.

Remark 4.16. We have in fact established that with the notation in (4.29) and (4.30), the cohomology class of $\zeta^{1}(s)+\zeta^{2}(s)$ vanishes identically on its domain of definition.

\section{Double complexes and equivariant cohomology}

In this section, we consider the case of a compact complex Kähler manifold $X$ equipped with a holomorphic section $K$ of $T X$, which vanishes on a submanifold $Y$. We study the hypercohomology of the corresponding double complex. When $K_{\mathbb{R}}$ is Killing, we relate the double complex to the de Rham complex in equivariant cohomology.

This section is organized as follows. In $\S 5.1$, we introduce the geometric setting. In $\S 5.2$, we construct the complex of hypercohomology, and we recall results of [CaL73] on the degeneracy of the corresponding spectral sequence. In $\S 5.3$, we construct the associated Hodge theory, and we compare various Laplacians, which coincide when $K_{\mathbb{R}}$ is Killing. In $\S 5.4$, we use the Bott localization formulas to evaluate the $L_{2}$ metric on the hypercohomology. In $\S 5.5$, we derive a corresponding formula for the $L_{2}$ metric on the determinant of the hypercohomology. In $\S 5.6$, we give a corresponding formula for the equivariant determinant in the sense of [B95]. In $\S 5.7$, we specialize our results to the case where there is $f \in \mathfrak{g}$, the Lie algebra of $G$, such that $K$ is induced by $f$. In $\S 5.8$, when $K_{\mathbb{R}}$ is a Hamiltonian vector field, we construct an associated de Rham complex. In $\S 5.9$, we show that this complex is just a twisted de Rham complex as in [W82]. In $\S 5.10$, we consider the case of families of such manifolds. Finally in $\S 5.11$, we prove a conjugation formula relating the holomorphic LeviCivita superconnection for the hypercohomology to the de Rham Levi-Civita superconnection for the twisted Witten complex.

\subsection{A holomorphic vector field}

We use the same notation as in $\S 2.1$, and we suppose that the corresponding assumptions are in force, except that for the moment, we do not assume $X$ to be Kähler.

Let $K$ be a holomorphic section of $T X$. We assume that $K$ vanishes on a complex submanifold $Y \subset X$. We will assume that $\left.\nabla K\right|_{Y}:\left.N_{Y / X} \rightarrow T X\right|_{Y}$ is injective. Therefore $\left.\nabla K\right|_{Y}$ identifies $N_{Y / X}$ to the holomorphic subvector bundle $\left.\operatorname{Im} \nabla K\right|_{Y}$ of $T X$. We have the exact sequence

$$
\left.0 \longrightarrow T Y \longrightarrow T X\right|_{Y} \longrightarrow N_{Y / X} \longrightarrow 0
$$

In the sequel, we will assume that $\left.\operatorname{Im} \nabla K\right|_{Y}$ is transverse to $T Y$, so that

$$
\left.T X\right|_{Y}=\left.T Y \oplus \operatorname{Im} \nabla K\right|_{Y}
$$




\section{J.-M. Bismut}

By (5.1) and (5.2), the exact sequence (5.1) splits canonically, so that

$$
\left.T X\right|_{Y}=T Y \oplus N_{Y / X}
$$

Moreover $\left.\nabla K\right|_{Y}$ can be considered as an automorphism of $\left.\operatorname{Im} \nabla K\right|_{Y}$ or of $N_{Y / X}$.

The above assumptions are verified when $K$ is holomorphic and Killing. In this case, $N_{Y / X}$ can be identified to the orthogonal vector bundle to $T Y$ in $\left.T X\right|_{Y}$, and $\left.\nabla K\right|_{Y}$ is a skew-adjoint parallel section of $\operatorname{End}\left(N_{Y / X}\right)$.

Let $K_{\mathbb{R}}=K+\bar{K}$ be the section of $T_{\mathbb{R}} X$ corresponding to $K$. Put

$$
d_{K}^{X}=d^{X}+i_{K_{\mathbb{R}}}, \quad \bar{\partial}_{K}^{X}=\bar{\partial}^{X}+i_{K}, \quad \partial_{K}^{X}=\partial^{X}+i_{\bar{K}} .
$$

Clearly

$$
d_{K}^{X}=\bar{\partial}_{K}^{X}+\partial_{K}^{X}
$$

The operators in (5.4) act on $\Omega^{\bullet}(X, \mathbb{C})$. Since $K$ is holomorphic,

$$
\bar{\partial}_{K}^{X, 2}=0, \quad \partial_{K}^{X, 2}=0, \quad d_{K}^{X, 2}=\left[\bar{\partial}_{K}^{X}, \partial_{K}^{X}\right]=L_{K_{\mathbb{R}}} .
$$

We will use corresponding notation on the submanifold $Y$. In particular, the upper script $X$ will be replaced by $Y$.

\subsection{The complex $\left(\Omega^{\bullet}(X, \mathbb{C}), \bar{\partial}_{K}^{X}\right)$}

In this section, the $\mathbb{Z}$-grading on $\Omega^{\circ}(X, \mathbb{C})$ is defined by the operator $\mathcal{N}$. We will also consider the corresponding $\mathbb{Z}$-grading on $\Omega^{\bullet}(Y, \mathbb{C})$. Let $\mathcal{H}_{K}^{\cdot}(X, \mathbb{C})$ be the cohomology of $\left(\Omega^{\bullet}(X, \mathbb{C}), \bar{\partial}_{K}^{X}\right)$.

Recall that the decreasing filtration $F^{\bullet}$ on $\Omega^{\bullet}(X, \mathbb{C})$ was defined in $(2.10)$. Let $G^{\bullet}$ be the increasing filtration

$$
G^{p} \Omega^{\bullet}(X, \mathbb{C})=\bigoplus_{\substack{0 \leqslant p^{\prime} \leqslant p, 0 \leqslant q \leqslant \ell}} \Omega^{\left(p^{\prime}, q\right)}(X, \mathbb{C}) .
$$

Then $\left(\Omega^{\bullet}(X, \mathbb{C}), \bar{\partial}_{K}^{X}\right)$ is a $\mathbb{Z}$-graded complex, and $G^{\bullet}$ is a filtration on this complex.

Consider the spectral sequence associated to the filtration $G^{\bullet}$ on $\Omega^{\bullet}(X, \mathbb{C})$. The associated $E_{1}$ is given by

$$
E_{1}=H^{\bullet}\left(X, \Lambda^{\bullet}\left(T^{*} X\right)\right) .
$$

Similarly, the cohomology of $\left(\Omega^{\bullet}(Y, \mathbb{C}), \bar{\partial}^{Y}\right)$ is just $H^{\bullet}\left(Y, \Lambda^{\bullet}\left(T^{*} Y\right)\right)$.

Let $j: Y \rightarrow X$ be the obvious embedding.

Theorem 5.1. The map $j^{*}: \Omega^{\bullet}(X, \mathbb{C}) \rightarrow \Omega^{\bullet}(Y, \mathbb{C})$ induces a quasi-isomorphism of filtered complexes $\left(\Omega^{\bullet}(X, \mathbb{C}), \bar{\partial}_{K}^{X}\right) \rightarrow\left(\Omega^{\bullet}(Y, \mathbb{C}), \bar{\partial}^{Y}\right)$.

Proof. Since $K$ vanishes on $Y, j^{*}$ defines a chain map of filtered complexes. We will show it is a quasi-isomorphism.

First we replace the Dolbeault complexes by their corresponding Cech counterparts. Namely let $\delta^{X}, \delta^{Y}$ be the Čech operators acting on $X, Y$. Set $\delta_{K}^{X}=\delta^{X}+i_{K}$. We denote by $\left(\mathcal{O}(X), \delta_{K}^{X}\right)$ and by $\left(\mathcal{O}(Y), \delta^{Y}\right)$ the corresponding filtered complexes. Note here that $\mathcal{O}(X)$ is given by local holomorphic sections of $\Lambda^{\bullet}\left(T^{*} X\right)$, and $\mathcal{O}(Y)$ by local holomorphic sections of $\Lambda^{*}\left(T^{*} Y\right)$. Again $j^{*}$ is a chain map of filtered Čech complexes. We claim that this map is a quasi-isomorphism.

Let $F_{\delta}^{\cdot}$ be the obvious Čech filtration on the above double complexes. By $(5.2)$, the $\left(E_{1}, d_{1}\right)$ of the first complex with respect to the filtration $F_{\delta}^{*}$ is just $\left(\mathcal{O}(Y), \delta^{Y}\right)$. The $\left(E_{1}, d_{1}\right)$ of the second complex is just itself, i.e. $\left(\mathcal{O}(Y), \delta^{Y}\right)$. Therefore $j^{*}$ induces the obvious identification of the $\left(E_{1}, d_{1}\right)$. A standard result in homological algebra [CE56, ch. XIII, Theorem 3.2] shows that $j^{*}$ is a quasi-isomorphism. 


\section{HolOMORPHIC AND DE RHAM TORSION}

Let us now establish our theorem. We replace the complex $\left(\mathcal{O}(X), \delta_{K}^{X}\right)$ by its Dolbeault resolution. This new complex has now three partial differentials: $\bar{\partial}^{X}, \delta^{X}$ and $i_{K}$, which anticommute. Needless to say, we equip this complex with the full differential $\bar{\partial}^{X}+\delta^{X}+i_{K}$. When $K=0$, a standard spectral sequence argument shows that the cohomology of $\left(\Omega^{\bullet}(X, \mathbb{C}), \bar{\partial}^{X}\right)$ and $\left(\mathcal{O}(X), \delta^{X}\right)$ are canonically identified.

We then find that the chain map $j^{*}:\left(\Omega^{\bullet}(X, \mathbb{C}), \bar{\partial}_{K}^{X}\right) \rightarrow\left(\Omega^{\bullet}(Y, \mathbb{C}), \bar{\partial}^{Y}\right)$ is homotopic to the chain map $i_{*}:\left(\mathcal{O}(X), \delta_{K}^{X}\right) \rightarrow\left(\mathcal{O}(Y), \delta^{Y}\right)$. Since this latter map is a quasi-isomorphism, the first map is also a quasi-isomorphism. The proof of our theorem is completed.

Remark 5.2. By (5.8), $H^{\bullet}\left(X, \Lambda^{\bullet}\left(T^{*} X\right)\right)$ is the $E_{1}$-term of a spectral sequence which abuts to $\mathcal{H}_{K}^{\cdot}(X, \mathbb{C})$. By Theorem 5.1,

$$
\mathcal{H}_{K}^{\cdot}(X, \mathbb{C}) \simeq H^{\bullet}\left(Y, \Lambda^{\bullet}\left(T^{*} Y\right)\right)
$$

Observe that if $Y$ is Kähler, then $H^{\bullet}\left(Y, \Lambda^{\bullet}\left(T^{*} Y\right)\right) \simeq H^{\bullet}(Y, \mathbb{C})$. Therefore, when $Y$ is Kähler,

$$
\mathcal{H}_{K}^{\cdot}(X, \mathbb{C}) \simeq H^{\bullet}(Y, \mathbb{C}) \text {. }
$$

Note that $H^{\bullet}(Y, \mathbb{C})$ is equipped with an obvious conjugation operator. By $(5.9), \mathcal{H}_{K}^{\cdot}(X, \mathbb{C})$ is then equipped with a corresponding conjugation operator.

Note that by an argument due to Carrell and Liebermann [CaL73], which uses the Deligne degeneracy criterion [D68], if $X$ is connected and Kähler and if $Y$ is non-empty, the above spectral sequence degenerates at $E_{1} \simeq H^{\bullet}(X, \mathbb{C})$. In this case,

$$
E_{1} \simeq \operatorname{Gr}_{K}^{\cdot}(X, \mathbb{C})
$$

However in general the identification (5.10) does not preserve the $G$ filtration, i.e. the map $j^{*}$ : $H^{\bullet}(X, \mathbb{C}) \rightarrow H^{\bullet}(Y, \mathbb{C})$ is not an isomorphism.

\subsection{The adjoint operators}

We make the same assumptions as in $\S 2.1$. In particular, we still denote by $\omega^{X}$ the Kähler form of $X$. We equip $\Omega^{\bullet}(X, \mathbb{C})$ with the metric (2.5). We denote with a ${ }^{*}$ the formal adjoint of the above operators with respect to $(2.5)$.

Recall that $K_{\mathbb{R}}^{*}=\bar{K}^{*}+K^{*} \in T_{\mathbb{R}}^{*} X$ corresponds to $K_{\mathbb{R}}=K+\bar{K} \in T_{\mathbb{R}} X$. Note here that $\bar{K}^{*} \in T^{*} X$ corresponds to $\bar{K} \in \overline{T X}$, and $K^{*} \in \overline{T^{*} X}$ corresponds to $K \in T X$.

Then

$$
d_{K}^{X *}=d^{X, *}+K_{\mathbb{R}}^{*} \wedge, \quad \bar{\partial}_{K}^{X *}=\bar{\partial}^{X, *}+\bar{K}^{*} \wedge, \quad \partial_{K}^{X *}=\partial^{X, *}+K^{*} \wedge .
$$

By (5.6),

$$
d_{K}^{X *, 2}=\left[\bar{\partial}_{K}^{X *}, \partial_{K}^{X *}\right]=L_{K_{\mathbb{R}}}^{*} .
$$

Recall that we define $R$ as in (1.20) with respect to the fibrewise symplectic form $\omega^{X}$.

Theorem 5.3. The following identities hold:

$$
\bar{\partial}_{K}^{X *}=-i\left[\partial_{K}^{X}, R\right], \quad \partial_{K}^{X *}=i\left[\bar{\partial}_{K}^{X}, R\right] .
$$

Moreover,

$$
\begin{array}{r}
{\left[\bar{\partial}_{K}^{X}, \partial_{K}^{X *}\right]=0, \quad\left[\partial_{K}^{X}, \bar{\partial}_{K}^{X *}\right]=0, \quad\left[\bar{\partial}_{K}^{X}, \bar{\partial}_{K}^{X *}\right]-\left[\partial_{K}^{X}, \partial_{K}^{X *}\right]=-i\left[L_{K_{\mathbb{R}}}, R\right],} \\
\left(d_{K}^{X}+d_{K}^{X *}\right)^{2}=L_{K_{\mathbb{R}}}+L_{K_{\mathbb{R}}}^{*}+\left[d_{K}^{X}, d_{K}^{X *}\right], \quad\left[d_{K}^{X}, d_{K}^{X *}\right]=\left[\bar{\partial}_{K}^{X}, \bar{\partial}_{K}^{X *}\right]+\left[\partial_{K}^{X}, \partial_{K}^{X *}\right] .
\end{array}
$$

Proof. Since $\omega^{X}$ is closed, in (2.7), we can as well replace $\Lambda$ by $R$. Equation (5.14) is now trivial. The first two identities in (5.15) now follow from (5.14). Also using (5.6) and (5.14), we get

$$
\begin{gathered}
{\left[\bar{\partial}_{K}^{X}, \bar{\partial}_{K}^{X *}\right]=-i\left[\bar{\partial}_{K}^{X},\left[\partial_{K}^{X}, R\right]\right]=-i\left[L_{K_{\mathbb{R}}}, R\right]+i\left[\partial_{K}^{X},\left[\bar{\partial}_{K}^{X}, R\right]\right],} \\
{\left[\partial_{K}^{X}, \partial_{K}^{X *}\right]=i\left[\partial_{K}^{X},\left[\bar{\partial}_{K}^{X}, R\right]\right] .}
\end{gathered}
$$




\section{J.-M. Bismut}

From (5.16), we get the third identity in (5.15). From (5.6) and (5.13), we get the fourth identity in (5.15). Using the first two identities in (5.15), we obtain the last identity in (5.15). The proof of our theorem is completed.

\subsection{The $L_{2}$ metric on $\mathcal{H}_{K}^{\cdot}(X, \mathbb{C})$ and the Bott localization formulas} Set

$$
\mathcal{H}^{X}=\operatorname{ker} \bar{\partial}_{K}^{X} \cap \operatorname{ker} \bar{\partial}_{K}^{X *}
$$

Then $\mathcal{H}^{X}$ is a vector subspace of $\Omega^{\bullet}(X, \mathbb{C})$. By Hodge theory,

$$
\mathcal{H}_{K}^{\cdot}(X, \mathbb{C}) \simeq \mathcal{H}^{X}
$$

As a vector subspace of $\Omega^{\bullet}(X, \mathbb{C}), \mathcal{H}^{X}$ inherits a metric from the metric $(2.5)$ on $\Omega^{\bullet}(X, \mathbb{C})$. Let $h^{\mathcal{H}_{K}^{*}(X, \mathbb{C})}$ denote the corresponding metric on $\mathcal{H}_{K}^{\cdot}(X, \mathbb{C})$.

Observe that $\mathcal{H}_{K}^{\cdot}(X, \mathbb{C})$ is an algebra, which is $\mathbb{Z}$-graded by $\mathcal{N}$. Also if $\alpha \in \mathcal{H}_{K}^{\cdot}(X, \mathbb{C})$, and if $\beta \in \Omega^{\bullet}(X, \mathbb{C})$ represents $\alpha$, then $\int_{X} \beta$ depends only on $\alpha$. From now on, we will write $\int_{X} \alpha$ instead of $\int_{X} \beta$.

Recall that as we saw in $\S 5.1,\left.\nabla K\right|_{Y}$ can be considered as a holomorphic section of $\operatorname{End}\left(N_{Y / X}\right)$. Let $h^{N_{Y / X}}$ be a Hermitian metric on $N_{Y / X}$, let $\nabla^{N_{Y / X}}$ be the corresponding holomorphic Hermitian connection, and let $R^{N_{Y / X}}$ be its curvature. Set

$$
R_{K}^{N_{Y / X}}=R^{N_{Y / X}}-2 i \pi \nabla^{T X} K
$$

Definition 5.4. Set

$$
c_{K}\left(N_{Y / X}, \nabla^{N_{Y / X}}\right)=\operatorname{det}\left(-R_{K}^{N_{Y / X}} / 2 i \pi\right) .
$$

Then $c_{K}\left(N_{Y / X}, \nabla^{N_{Y / X}}\right)$ is an even form, which is $\bar{\partial}^{Y}$ closed. Let $c_{K}\left(N_{Y / X}\right) \in H^{\bullet}\left(Y, \Lambda^{\bullet}\left(T^{*} Y\right)\right) \simeq$ $H^{\bullet}(Y, \mathbb{C})$ be the corresponding cohomology class.

Now we recall a formula of [Bo67] when $Y$ consists of a collection of points, which was extended by Zhang [Z90] under the transversality assumption given in (5.2). Note that, if $K_{\mathbb{R}}$ is a Killing vector field, then our formula is known as the localization formula of [DuH82, DuH83, BeV83] in equivariant cohomology.

Theorem 5.5. If $\alpha \in H_{K}^{\cdot}(X, \mathbb{C})$, then

$$
\int_{X} \alpha=\int_{Y} \frac{\alpha}{c_{-K / 2 i \pi}\left(N_{Y / X}\right)}
$$

Take $\alpha, \beta \in \mathcal{H}_{K}^{\cdot}(X, \mathbb{C})$. Then $\alpha \beta \in \mathcal{H}_{K}^{\cdot}(X, \mathbb{C})$. By Theorem 5.5 , we get

$$
\int_{X} \alpha \beta=\int_{Y} \frac{\alpha \beta}{c_{-K / 2 i \pi}\left(N_{Y / X}\right)} .
$$

Now recall that by $(5.9), \mathcal{H}_{K}^{\cdot}(X, \mathbb{C}) \simeq H^{\bullet}\left(Y, \Lambda^{\bullet}\left(T^{*} Y\right)\right)$, and this identification is an identification of $\mathbb{Z}$-graded vector spaces. Moreover, by Serre duality,

$$
a, b \in H^{\bullet}\left(Y, \Lambda^{\bullet}\left(T^{*} Y\right)\right) \rightarrow \int_{Y} \frac{a b}{c_{-K / 2 i \pi}\left(N_{Y / X}\right)}
$$

is a non-degenerate bilinear form. By (5.22), we deduce that the bilinear form

$$
\alpha, \beta \in \mathcal{H}_{K}^{\cdot}(X, \mathbb{C}) \rightarrow \int_{X} \alpha \beta
$$


is also non-degenerate. Observe that this last result also follows from (5.17). Indeed if $\alpha \in \mathcal{H}^{X}$, then $\overline{* \alpha} \in \mathcal{H}^{X}$, so that if $\alpha \neq 0$, then

$$
\int_{X} \alpha \wedge \overline{* \alpha}>0
$$

\subsection{The $L_{2}$ metric on the determinant of the cohomology}

We make the same assumptions as in $\S 5.4$.

If $E$ is a finite-dimensional complex vector space, set $\operatorname{det} E=\Lambda^{\max }(E)$. If $\lambda$ is a complex line, let $\lambda^{-1}$ be the associated dual line. If $E=\bigoplus_{1 \leqslant i \leqslant m} E^{i}$ is a $\mathbb{Z}$-graded complex vector space, set

$$
\operatorname{det} E=\bigotimes_{1 \leqslant i \leqslant m}\left(\operatorname{det} E^{i}\right)^{(-1)^{i}}
$$

Definition 5.6. Put

$$
\lambda_{K}^{X}=\operatorname{det} \mathcal{H}_{K}^{\cdot}(X, \mathbb{C}), \quad \lambda^{X}=\operatorname{det} H^{\bullet}(X, \mathbb{C}), \quad \lambda^{Y}=\operatorname{det} H^{\bullet}(Y, \mathbb{C}) .
$$

Note that using Serre duality, we find that $\lambda^{X, 2}$ and $\lambda^{Y, 2}$ are canonically trivial. By (5.9), we have a canonical isomorphism

$$
\lambda_{K}^{X} \simeq \lambda^{Y}
$$

Also observe that, as we saw at the end of $\S 5.2$, if $X$ is connected and if $Y$ is non-empty,

$$
\lambda_{K}^{X} \simeq \lambda^{X} .
$$

The lines $\lambda_{K}^{X}, \lambda^{X}, \lambda^{Y}$ inherit metrics ||$_{\lambda_{K}^{X}},||_{\lambda^{X}}, \|_{\lambda^{Y}}$ from the given metrics $h^{\mathcal{H}_{K}^{\bullet}(X, \mathbb{C})}, h^{H^{\bullet}(X, \mathbb{C})}$ and $h^{H^{\bullet}(Y, \mathbb{C})}$. Note that the metrics ||$_{\lambda^{X}},||_{\lambda^{Y}}$ are canonically trivial on $\lambda^{X}, \lambda^{Y}$.

We claim that these metrics do not depend on $\omega^{X}$. In fact, since the antilinear map $\bar{*}$ is an isometry of $\mathcal{H}^{X}$, the metric on $\lambda_{K}^{X}$ only depends on the non-degenerate bilinear form (5.24) on $\mathcal{H}_{K}^{\cdot}(X, \mathbb{C})$ which is metric independent. The same considerations apply to the metrics on $\lambda^{X}, \lambda^{Y}$, which we already know are canonically trivial.

Note that since $\left.\nabla K\right|_{Y}$ is a holomorphic section of $\operatorname{End}\left(N_{Y / X}\right)$, $\operatorname{det}\left(\left.\nabla K\right|_{Y}\right)$ is a holomorphic function on $Y$. Since $Y$ is compact, this function is locally constant.

THEOREM 5.7. The following identity holds:

$$
\log \left(\frac{||_{\lambda_{K}^{X}}}{\|\left.\right|_{\lambda^{Y}}}\right)^{2}=-\int_{Y} c_{\max }(T Y) \log \left|\operatorname{det}\left(\left.\nabla^{T X} K\right|_{N_{Y / X}}\right)\right|
$$

Proof. Clearly the antilinear operator $\bar{*}$ maps $\mathcal{H}^{X}$ into itself as an isometry. The same considerations apply to $H^{\bullet}(Y, \mathbb{C})$. Recall that $X$ has complex dimension $\ell$. Let $\ell^{\prime}$ be the complex dimension of $Y$. To evaluate the left-hand side of (5.30), we only need to compare the bilinear forms

$$
\alpha, \beta \in \mathcal{H}_{K}^{\cdot}(X, \mathbb{C}) \rightarrow \frac{1}{(2 \pi)^{\ell}} \int_{X} \alpha \beta
$$

and

$$
a, b \in H^{\bullet}(Y, \mathbb{C}) \rightarrow \frac{1}{(2 \pi)^{\ell^{\prime}}} \int_{Y} a b .
$$

Let $A_{K} \in \operatorname{End}^{\text {even }}\left(H^{\bullet}(Y, \mathbb{C})\right)$ be given by

$$
a \rightarrow \frac{a}{(2 \pi)^{\ell-\ell^{\prime}} c_{-K / 2 i \pi}\left(N_{Y / X}\right)} .
$$


Set

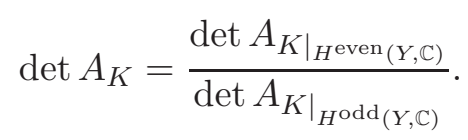

Then

$$
\left(\frac{\left.\right|_{\lambda_{K}^{X}}}{\|_{\lambda^{Y}}}\right)^{2}=\left|\operatorname{det} A_{K}\right|
$$

Now we observe that $A_{K}$ is an operator which increases the degree in $H^{\bullet}(Y, \mathbb{C})$. Therefore it acts like a triangular matrix, whose diagonal part is just multiplication by the locally constant function $1 / \operatorname{det}\left(\left.i \nabla^{T X} K\right|_{N_{Y / X}}\right)$. Using (5.34), we get

$$
\operatorname{det} A_{K}=\left(1 / \operatorname{det}\left(\left.i \nabla^{T X} K\right|_{N_{Y / X}}\right)\right)^{\chi(Y)} \text {. }
$$

By (5.35) and (5.36) and by the Chern-Gauss-Bonnet theorem, we get (5.30). The proof of our theorem is completed.

\subsection{The $L_{2}$ metric on the equivariant determinant}

Let $G$ be a compact Lie group acting holomorphically on $X$ and preserving the Kähler form $\omega^{X}$. Also we assume that $K$ is $G$-invariant. Then $G$ also acts on $Y$.

Let $G_{O} \subset G$ be the connected component of the identity. Then $G_{O}$ acts trivially on $\mathcal{H}_{K}(X, \mathbb{C})$ and on $H^{\bullet}(Y, \mathbb{C})$. We can identify $\mathcal{H}_{K}^{\cdot}(X, \mathbb{C})$ and $H^{\bullet}(Y, \mathbb{C})$ as representation spaces of $G / G_{o}$.

For the definition of equivariant determinants and of corresponding metrics, we refer to [B95]. Let us just say that the equivariant determinant is obtained by splitting the cohomology into its irreducible pieces under the action of $G$, and by taking the direct sum of the determinants of these irreducible pieces. In the sequel, $\lambda_{K}^{X}, \lambda^{Y}$ denote the equivariant determinants of $\mathcal{H}^{\bullet}(X, \mathbb{C}), H^{\bullet}(Y, \mathbb{C})$, and ||$_{\lambda_{K}^{X}},\left.\right|_{\lambda^{Y}}$ denote the corresponding $L_{2}$ metrics.

TheOrem 5.8. For any $g \in G$,

$$
\log \left(\frac{||_{\lambda_{K}^{X}}}{\|\left.\right|_{\lambda^{Y}}}\right)^{2}(g)=-\int_{Y_{g}} c_{\max }\left(T Y_{g}\right) \log \left|\operatorname{det}\left(\left.\nabla^{T X} K\right|_{N_{Y / X}}\right)\right| .
$$

Proof. We proceed as in the proof of Theorem 5.7. The operator $A_{K}$ defined in (5.33) commutes with $g$. We get the easy formula,

$$
\log \left(\frac{||_{\lambda_{K}^{X}}}{\|\left.\right|_{\lambda^{Y}}}\right)^{2}(g)=\operatorname{Tr}_{\mathrm{S}}{ }^{\bullet}(Y, \mathbb{C})\left[g \log \left|A_{K}\right|\right] .
$$

The operator $g$ preserves the degree, while $A_{K}$ increases the degree in $H^{\bullet}(Y, \mathbb{C})$. By $(5.38)$, we get

$$
\log \left(\frac{||_{\lambda_{K}^{X}}}{||_{\lambda^{Y}}}\right)^{2}(g)=-\operatorname{Tr}_{\mathrm{s}}{ }^{H^{*}(Y, \mathbb{C})}\left[g \log \left|\operatorname{det}\left(\left.\nabla K\right|_{N_{Y / X}}\right)\right|\right] .
$$

Using (5.39) and the Lefschetz formula, we get (5.37).

\subsection{The case where $K=f^{X}$}

We make the same assumptions as in $\S 5$.6. Let $\mathfrak{g}$ be the Lie algebra of $G$. If $f \in \mathfrak{g}$, let $f^{X} \in T X$ be the vector field on $X$ which is associated to $f$. 


\section{HolOMORPHIC AND DE RHAM TORSION}

In the sequel, we assume that there is $f \in \mathfrak{g}$, which is $G$-invariant, such that $K=f^{X}$. Then $K$ is a holomorphic section of $T X$, and $K_{\mathbb{R}}$ is a Killing vector field. Set

$$
\Omega_{K}(X, \mathbb{R})=\left\{s \in \Omega^{\bullet}(X, \mathbb{R}), L_{K} s=0\right\} .
$$

Then the operator $d_{K}^{X}$ acts as an odd operator on $\Omega_{K}^{*}(X, \mathbb{R})$, and moreover,

$$
\left.d_{K}^{X, 2}\right|_{\Omega_{K}^{\bullet}(X, \mathbb{R})}=0 .
$$

The cohomology of the complex $\left(\Omega_{K}^{\bullet}(X, \mathbb{R}), d_{K}^{X}\right)$ will be denoted by $H_{K}^{\cdot}(X, \mathbb{R})$. It splits as

$$
H_{K}^{\bullet}(X, \mathbb{R})=H_{K}^{\text {even }}(X, \mathbb{R}) \oplus H_{K}^{\text {odd }}(X, \mathbb{R}) .
$$

Now we recall a result stated in [W82].

Theorem 5.9. The chain map $j^{*}:\left(\Omega_{K}^{*}(X, \mathbb{R}), d_{K}^{X}\right) \rightarrow\left(\Omega^{\bullet}(Y), d^{Y}\right)$ is a quasi-isomorphism of $\mathbb{Z}_{2}$-graded complexes.

By Theorem 5.9, we deduce the canonical isomorphism of $\mathbb{Z}_{2}$-graded vector spaces

$$
H_{K}^{\cdot}(X, \mathbb{R}) \simeq H^{\bullet}(Y, \mathbb{R}) .
$$

Proposition 5.10. The following identity holds:

$$
\left(d_{K}^{X}+d_{K}^{X *}\right)^{2}=\left[d_{K}^{X}, d_{K}^{X *}\right]=2\left[\bar{\partial}_{K}^{X}, \bar{\partial}_{K}^{X *}\right]=2\left[\partial_{K}^{X}, \partial_{K}^{X *}\right] .
$$

Proof. Since $K$ is Killing,

$$
L_{K_{\mathbb{R}}}^{*}=-L_{K_{\mathbb{R}}}, \quad\left[L_{K_{\mathbb{R}}}, R\right]=0 .
$$

Our proposition now follows from Theorem 5.3 and from (5.45).

Recall that formula (5.5) relates $d_{K}^{X}, \bar{\partial}_{K}^{X}$ and $\partial_{K}^{X}$. It is then clear that $d_{K}^{X}$ is not compatible with any of the above filtrations.

Set

$$
\mathcal{K}^{X}=\operatorname{ker}\left(d_{K}^{X}+d_{K}^{X *}\right)^{2} .
$$

Observe that by (5.44),

$$
\mathcal{K}^{X}=\operatorname{ker} d_{K}^{X} \cap \operatorname{ker} d_{K}^{X *} .
$$

By (5.6) and (5.47), we find that ker $\mathcal{K}^{X}$ consists of $K$-invariant forms. Using Hodge theory, we then recover the result of Witten [W82] that

$$
\mathcal{K}^{X} \simeq H_{K}^{\cdot}(X, \mathbb{R})
$$

By (5.44), we get

$$
\mathcal{K}^{X} \otimes_{\mathbb{R}} \mathbb{C}=\mathcal{H}^{X}
$$

By (5.48) and (5.49), we see that

$$
\mathcal{H}_{K}^{\cdot}(X, \mathbb{C}) \simeq H_{K}^{\cdot}(X, \mathbb{C})
$$

Note that (5.50) seems to depend on the choice of $\omega^{X}$. However observe that the identifications in (5.50) are compatible with the identification of both sides of (5.50) to $H^{\bullet}(Y, \mathbb{C})$. Therefore the identification in (5.50) is indeed canonical.

We will denote by $h^{H_{K}^{*}(X, \mathbb{R})}$ the metric on $H_{K}^{\cdot}(X, \mathbb{R})$ which one obtains via the identification (5.48). Note that by (5.18), (5.49) and (5.50), this is the same as the metric $h^{\mathcal{H}_{K}^{*}(X, \mathbb{C})}$. 


\section{J.-M. Bismut}

As was observed in Remark 5.2, since $Y$ is Kähler, there is a natural conjugation operator acting on $\mathcal{H}_{K}(X, \mathbb{C})$. Because of $(5.50)$, the existence of this conjugation operator is now obvious. Finally observe that the operator $\mathcal{N}$ refines on the $\mathbb{Z}_{2}$-grading of $H_{K}^{\cdot}(X, \mathbb{C})$.

Let $d^{Y *}$ be the formal adjoint of $d^{Y}$. Set

$$
\mathcal{K}^{Y}=\operatorname{ker}\left(d^{Y}+d^{Y *}\right)^{2} .
$$

Then by Hodge theory,

$$
\mathcal{K}^{Y} \simeq H^{\bullet}(Y, \mathbb{R})
$$

\subsection{A real flat line bundle}

We make the same assumptions as in $\S 5.7$. Set

$$
\gamma_{K}=-i_{K_{\mathbb{R}}} \omega^{X}
$$

Since $K_{\mathbb{R}}$ preserves the Kähler form $\omega^{X}$, then

$$
d^{X} \gamma_{K}=0
$$

Set

By (5.54), we get

$$
d_{\gamma_{K}}^{X}=d^{X}+\gamma_{K}
$$

$$
d_{\gamma_{K}}^{X, 2}=0 .
$$

The operator $d_{\gamma_{K}}^{X}$ can be considered as the de Rham operator acting on the de Rham complex with values in a flat real line bundle with connection form $d_{\gamma_{K}}^{X}$. Let $d_{\gamma_{K}}^{X, *}$ be the formal adjoint of $d_{\gamma_{K}}^{X}$. Then

$$
d_{\gamma_{K}}^{X, *}=d^{X, *}+i_{J^{T} \mathbb{R} X} K_{\mathbb{R}} .
$$

Let $H_{\gamma_{K}}^{\cdot}(X, \mathbb{R})$ be the cohomology of the complex $\left(\Omega^{\bullet}(X, \mathbb{R}), d_{\gamma_{K}}^{X}\right)$. We will now consider the corresponding Hodge theory. Namely, set

$$
\mathcal{L}^{X}=\operatorname{ker} d_{\gamma_{K}}^{X} \cap \operatorname{ker} d_{\gamma_{K}}^{X, *} .
$$

Then

$$
H_{\gamma_{K}}^{\cdot}(X, \mathbb{R}) \simeq \mathcal{L}^{X}
$$

THEOREM 5.11. The following identities hold:

$$
\begin{aligned}
& e^{-\pi M / 4} i^{N^{(1,0)}} \sqrt{2} d_{\gamma_{K}}^{X} i^{-N^{(1,0)}} e^{\pi M / 4}=\bar{\partial}_{K}^{X}+\bar{\partial}_{K}^{X *}+i\left(\partial_{K}^{X}+\partial_{K}^{X *}\right), \\
& e^{-\pi M / 4} i^{N^{(1,0)}} \sqrt{2} d_{\gamma_{K}}^{X, *} i^{-N^{(1,0)}} e^{\pi M / 4}=\bar{\partial}_{K}^{X}+\bar{\partial}_{K}^{X *}-i\left(\partial_{K}^{X}+\partial_{K}^{X *}\right) .
\end{aligned}
$$

In particular,

$$
e^{-\pi M / 4} i^{N^{(1,0)}} \frac{\sqrt{2}}{2}\left(d_{\gamma_{K}}^{X}+d_{\gamma_{K}}^{X, *}\right) i^{-N^{(1,0)}} e^{\pi M / 4}=\bar{\partial}_{K}^{X}+\bar{\partial}_{K}^{X *}
$$

Proof. If $K=0$, this is just Theorem 3.5 when the base $S$ is reduced to a point. In the general case, we use Theorem 1.5, together with the fact that

$$
\bar{K}^{*} \wedge+i_{K}=i \frac{c_{T X}}{\sqrt{2}}\left(J^{T_{\mathbb{R}} X} K_{\mathbb{R}}\right), \quad i\left(K^{*} \wedge+i \bar{K}\right)=\frac{c_{\overline{T X}}}{\sqrt{2}}\left(J^{T_{\mathbb{R}} X} K_{\mathbb{R}}\right) .
$$




\section{HolOMORPHIC AND DE RHAM TORSION}

By Theorem 5.11, we deduce in particular that the map

$$
s \in \Omega^{\bullet}(X, \mathbb{C}) \rightarrow e^{-\pi M / 4} i^{N^{(1,0)}} s \in \Omega^{\bullet}(X, \mathbb{C})
$$

induces an isomorphism from $\mathcal{L}^{X}$ into $\mathcal{K}^{X}$. By (5.48) and (5.49), we find that this map induces an identification of $H_{\gamma_{K}}^{\cdot}(X, \mathbb{C})$ with $H_{K}^{\cdot}(X, \mathbb{C}) \simeq \mathcal{H}_{K}^{\cdot}(X, \mathbb{C})$. By (5.10) and (5.43), we know that

$$
H_{K}^{\cdot}(X, \mathbb{C}) \simeq \mathcal{H}_{K}^{\cdot}(X, \mathbb{C}) \simeq H^{\bullet}(Y, \mathbb{C}) .
$$

By (5.63), we deduce that there is an isomorphism of $\mathbb{Z}_{2}$-graded vector spaces,

$$
H_{\gamma_{K}}^{\bullet}(X, \mathbb{R}) \simeq H^{\bullet}(Y, \mathbb{R})
$$

\subsection{The case where the action is Hamiltonian}

We still make the same assumptions as in $\S 5.7$. Assume that $K_{\mathbb{R}}$ is a Hamiltonian vector field. Namely we assume that there is a smooth function $\mu_{K}: X \rightarrow \mathbb{R}$ such that

$$
d^{X} \mu_{K}=\gamma_{K}
$$

Then

By (5.66), we deduce that

$$
d_{\gamma_{K}}^{X}=e^{-\mu_{K}} d^{X} e^{\mu_{K}}
$$

$$
H_{\gamma_{K}}^{\bullet}(X, \mathbb{R}) \simeq H^{\bullet}(X, \mathbb{R}) .
$$

Recall that the increasing filtration $G^{\bullet}$ on $\Omega^{\bullet}(X, \mathbb{C})$ was defined in (5.7). Also, by (5.8),

$$
E_{1} \simeq H^{\bullet}(X, \mathbb{C}) .
$$

Theorem 5.12. The spectral sequence associated to the filtration $G^{\bullet}$ degenerates at $E_{1}$.

Proof. By (5.64) and (5.67), we get

$$
\operatorname{dim} H^{\text {even }}(X, \mathbb{R})=\operatorname{dim} H^{\text {even }}(Y, \mathbb{R}), \quad \operatorname{dim} H^{\text {odd }}(X, \mathbb{R})=\operatorname{dim} H^{\text {odd }}(Y, \mathbb{R}) .
$$

Now $E_{1}$ is the first term of a spectral sequence which abuts to $H^{\bullet}(Y, \mathbb{C})$. Using $(5.68)$ and $(5.69)$, this is only possible if the differential $d_{1}$ and the successive differentials vanish identically. Therefore the spectral sequence degenerates at $E_{1}$.

Remark 5.13. We already mentioned in Remark 5.2 that by a result of [CaL73], if $K$ is just holomorphic, if $X$ is connected and if $Y$ is non-empty, the spectral sequence associated to $G^{\bullet}$ degenerates at $E_{1}$. Under the assumptions of Theorem 5.12, $Y$ is necessarily non-empty, so that Theorem 5.12 follows from [CaL73].

Conversely, if $X$ is connected and if $Y$ is non-empty, Lichnerowicz's lemma [GrHa78, ch. 5, p. 711] asserts that there exists a smooth function $\nu_{K}: X \rightarrow \mathbb{C}$ such that

$$
\bar{\partial}^{X} \nu_{K}=-i_{K} \omega^{X}
$$

By conjugating (5.70), we get

By (5.70) and (5.71), we find that

$$
\partial^{X} \bar{\nu}_{K}=-i \bar{K}^{X} \omega^{X}
$$

$$
\gamma_{K}=\bar{\partial}^{X} \nu_{K}+\partial^{X} \bar{\nu}_{K}
$$

If $K=f^{X}$ as in Theorem 5.12, the form $\gamma_{K}$ is closed. By Hodge theory, we then find that a function $\mu_{K}: X \rightarrow \mathbb{R}$ exists, such that (5.65) holds. So Theorem 5.12 is indeed a consequence of [CaL73]. 


\section{J.-M. Bismut}

Take $\alpha \in H^{(p, q)}(X, \mathbb{C})$. By Theorem 5.12, we can find a smooth form

$$
\mathfrak{a} \in \bigoplus_{\substack{p^{\prime}-q^{\prime}=p-q \\ p^{\prime} \leqslant p}} \Omega^{\left(p^{\prime}, q^{\prime}\right)}(X)
$$

such that

$$
\bar{\partial}_{K}^{X} \mathfrak{a}=0,
$$

and that the component of $\mathfrak{a}$ which has degree $(p, q)$ is $\bar{\partial}^{X}$ closed and represents $\alpha$ in $\Omega^{(p, q)}(X, \mathbb{C})$.

We now claim that we can use (5.22) to localize the computation of intersection products on $Y$. Indeed take $\alpha \in H^{(p, q)}(X, \mathbb{C}), \beta \in H^{(\ell-p, \ell-q)}(X, \mathbb{C})$, and let $\mathfrak{a}, \mathfrak{b}$ be associated to $\alpha, \beta$ as before. Clearly,

$$
\int_{X} \alpha \beta=\int_{X} \mathfrak{a b}
$$

Using (5.22) and (5.74), we obtain a formula for $\int_{X} \alpha \beta$ which is localized on $Y$.

\subsection{An equivariant fibration}

We make the same assumptions as in $\S 4$, and we use the notation of $\S \S 2-5$. Also $K$ denotes a holomorphic $G$-invariant section of $T X$. The notation of $\S 5$ will be used either on $M$ or on $X$.

Consider the operator $d_{K}^{M}$. Then by Proposition 2.3,

$$
d_{K}^{M}=d_{K}^{X}+\nabla^{\Omega^{\bullet}(X, \mathbb{R})}+i_{T^{H}} .
$$

Similarly, by Theorem 2.15,

$$
d_{K}^{M}=d_{K}^{X}+\nabla^{\Omega^{\bullet}(X, \mathbb{R}), u}+i_{T} .
$$

The obvious analogue of Theorem 2.15 holds, when introducing the subscript $K$. The same is true for the analogue of Theorem 2.21 .

We can define $B_{K, t}^{\prime \prime}, B_{K, t}^{\prime}, B_{K, t}$ as in (3.4). The obvious analogue of (3.5) is now

$$
\begin{aligned}
& B_{K, t}^{\prime \prime}=e^{-i \omega^{H} / 2 t} t^{\mathcal{N} / 2}\left(\bar{\partial}_{K}^{X}+\nabla^{\Omega^{\bullet}(X, \mathbb{C}), u^{\prime \prime}}\right) t^{-\mathcal{N} / 2} e^{i \omega^{H} / 2 t}, \\
& B_{K, t}^{\prime}=e^{i \omega^{H} / 2 t} t^{-\mathcal{N} / 2}\left(\bar{\partial}_{K}^{X *}+\nabla^{\Omega^{\bullet}(X, \mathbb{C}), u^{\prime}}\right) t^{\mathcal{N} / 2} e^{-i \omega^{H} / 2 t} .
\end{aligned}
$$

Instead of (3.7), we now set

$$
\mathcal{N}_{t}=\mathcal{N}+i \frac{\omega^{H}}{t}
$$

Then, when doing the proper replacements, and replacing in particular $N_{t}^{(0,1)}$ by $\mathcal{N}_{t}$, the obvious analogue of (3.8) still holds.

Using (5.10), we find that the fibres $\mathcal{H}_{K}^{\cdot}(X, \mathbb{C})$ patch into a smooth vector bundle on $S$, and moreover, we have the isomorphism of vector bundles on $S$ which are $\mathbb{Z}$-graded by $\mathcal{N}$,

$$
\mathcal{H}_{K}^{\cdot}(X, \mathbb{C}) \simeq H^{\cdot}(Y, \mathbb{C}) .
$$

Moreover $\mathcal{H}_{K}^{\cdot}(X, \mathbb{C})$ can be canonically equipped with a holomorphic structure $\nabla^{\mathcal{H}_{K}(X, \mathbb{C})^{\prime \prime}}$ similar to the one we constructed in Remark 2.22 for $H^{\bullet}(X, \mathbb{C})$. It can be computed using $\nabla^{\Omega^{\bullet}}(X, \mathbb{R}), u^{\prime \prime}$. The identification in (5.79) is then an identification of holomorphic $\mathbb{Z}$-graded vector bundles.

\subsection{The case where $K=f^{X}$}

In the sequel, we assume that there exists $f \in \mathfrak{g}$, which is $G$-invariant and such that $K=f^{X}$. In particular the metric $h^{T X}$ and the vector bundle $T^{H} M$ are $K_{\mathbb{R}}$-invariant. As in (5.53), set

$$
\gamma_{K}=-i_{K_{\mathbb{R}}} \omega^{X}
$$




\section{HolOMORPHIC AND DE RHAM TORSION}

Equivalently,

$$
\gamma_{K}=-i_{K_{\mathbb{R}}} \omega^{M}
$$

Then $\gamma_{K}$ is a 1 -form along the fibres $X$. Since $\omega^{M}$ is $K_{\mathbb{R}^{-}}$-invariant, the form $\gamma_{K}$ is closed on $M$.

We define $C_{\gamma_{K}, t}^{\dagger}, C_{\gamma_{K}, t}^{\dagger *}, C_{\gamma_{K}, t}, D_{\gamma_{K}, t}$ as in Definition 3.4 , by replacing $d^{X}, d^{X, *}$ by $d_{\gamma_{K}}^{X}, d_{\gamma_{K}}^{X, *}$. Then the obvious analogue of (3.13) holds. The analogue of (3.14) is now

$$
C_{\gamma_{K}, t}^{\dagger}=t^{N / 2} d_{\gamma_{K}}^{M} t^{-N / 2}
$$

From (5.82), we deduce in particular that

$$
C_{\gamma_{K}, t}^{\dagger 2}=0, \quad C_{\gamma_{K}, t}^{\dagger * 2}=0 .
$$

More generally, the obvious analogue of (3.15) holds.

TheOrem 5.14. For $t>0$, the following identities hold:

$$
\begin{aligned}
e^{-\pi M / 4} i^{N^{(1,0)}} C_{\gamma_{K}, 2 t}^{\dagger} i^{-N^{(1,0)}} e^{\pi M / 4}= & \sqrt{t}\left(\bar{\partial}_{K}^{X}+\bar{\partial}_{K}^{X *}+i\left(\partial_{K}^{X}+\partial_{K}^{X *}\right)\right)+\nabla^{\Omega^{\bullet}(X, \mathbb{C}), u} \\
& +i^{N^{(1,0)}}\left(\nabla^{\Omega^{\bullet}(X, \mathbb{C})}-\nabla^{\Omega^{\bullet}(X, \mathbb{C}), u}\right) i^{-N^{(1,0)}} \\
& -\frac{1}{2 \sqrt{2 t}}\left(c_{\overline{T X}}\left(T^{H}\right)-i c_{T X}\left(T^{H}\right)\right), \\
e^{-\pi M / 4} i^{N^{(1,0)}} C_{\gamma_{K}, 2 t}^{\dagger *} i^{-N^{(1,0)}} e^{\pi M / 4}= & \sqrt{t}\left(\bar{\partial}_{K}^{X}+\bar{\partial}_{K}^{X *}-i\left(\partial_{K}^{X}+\partial_{K}^{X *}\right)\right)+\nabla^{\Omega^{\bullet}(X, \mathbb{C}), u} \\
& -i^{N^{(1,0)}}\left(\nabla^{\Omega^{\bullet}(X, \mathbb{C})}-\nabla^{\Omega^{\bullet}(X, \mathbb{C}), u}\right) i^{-N^{(1,0)}} \\
& -\frac{1}{2 \sqrt{2 t}}\left(c_{\overline{T X}}\left(T^{H}\right)+i c_{T X}\left(T^{H}\right)\right) .
\end{aligned}
$$

In particular, for $t>0$,

$$
\begin{aligned}
e^{-\pi M / 4} i^{N^{(1,0)}} C_{\gamma_{K}, 2 t} i^{-N^{(1,0)}} e^{\pi M / 4}= & B_{K, t}, e^{-\pi M / 4} i^{N^{(1,0)}} D_{\gamma_{K}, 2 t} i^{-N^{(1,0)}} e^{\pi M / 4} \\
= & i\left(-\sqrt{t}\left(\partial_{K}^{X}+\partial_{K}^{X *}\right)+f^{\alpha} \bar{w}^{i} i_{T\left(f_{\alpha}, \bar{w}_{i}\right)}-\bar{f}^{\alpha} w^{i} i_{T\left(\bar{f}_{\alpha}, w_{i}\right)}\right. \\
& \left.-\frac{1}{2 \sqrt{2 t}} c_{T X}\left(T^{H}\right)\right) .
\end{aligned}
$$

Proof. This follows from Theorems 3.5 and 5.11.

By (5.43), we find that the $H_{K}^{\cdot}(X, \mathbb{R})$ patch into a smooth $\mathbb{Z}_{2}$-graded vector bundle on $S$, and moreover we have a canonical identification of vector bundles on $S$,

$$
H_{K}^{\cdot}(X, \mathbb{R}) \simeq H^{\bullet}(Y, \mathbb{R}) .
$$

Moreover since $K$ preserves all the geometric data, we get in particular,

$$
\left[\nabla^{\Omega^{\bullet}(X, \mathbb{R})}, d_{K}^{X}\right]=0 .
$$

Also the operator $L_{K_{\mathbb{R}}}$ preserves the horizontal and the vertical forms. Its action on $\Omega^{\bullet}(M, \mathbb{R})$ is given by

Also, by (2.23), we get

$$
L_{K_{\mathbb{R}}}=\left[d^{X}, i_{K_{\mathbb{R}}}\right]
$$

$$
\nabla^{\Omega^{\bullet}(X, \mathbb{R}), 2}+\left[d_{K}^{X}, i_{T^{H}}\right]=0
$$

By (5.87)-(5.89), we see that $H_{K}^{\cdot}(X, \mathbb{R})$ can be equipped with a flat Gauss-Manin connection $\nabla^{H_{K}^{*}(X, \mathbb{R})}$. Then (5.86) is an identification of flat vector bundles. 


\section{J.-M. Bismut}

Recall that $\mathcal{K}^{X}$ was defined in (5.47), and that by (5.48)-(5.50),

$$
\mathcal{H}_{K}^{\cdot}(X, \mathbb{C}) \simeq H_{K}^{\cdot}(X, \mathbb{C}) \simeq \mathcal{K}^{X} \otimes_{\mathbb{R}} \mathbb{C} .
$$

Then $\mathcal{K}^{X}$ is equipped with the $L_{2}$ metric in (2.5). Therefore the vector bundles $\mathcal{H}_{K}^{\cdot}(X, \mathbb{C})$ and $H_{K}^{\cdot}(X, \mathbb{R})$ inherit corresponding metrics $h^{\mathcal{H}_{K}^{\cdot}(X, \mathbb{C})}$ or $h^{H_{K}^{\cdot}(X, \mathbb{R})}$.

Let $P^{\mathcal{K}^{X}}$ be the orthogonal projection operator $\Omega^{\bullet}(X, \mathbb{R}) \rightarrow \mathcal{K}^{X}$.

DeFinition 5.15. Let $\nabla^{H_{K}^{\bullet}(X, \mathbb{R}), u}$ be the connection on $H^{\bullet}(X, \mathbb{R})$,

$$
\nabla^{H_{K}^{\bullet}(X, \mathbb{R}), u}=P^{\mathcal{K}^{X}} \nabla^{\Omega^{\bullet}(X, \mathbb{R}), u} .
$$

TheOREM 5.16. The connection $\nabla^{H_{K}^{*}(X, \mathbb{R}), u}$ preserves the metric $h^{H_{K}^{*}(X, \mathbb{R})}$. The following identity holds:

$$
\nabla^{H_{K}^{\bullet}(X, \mathbb{R}), u}=\nabla^{H_{K}^{\bullet}(X, \mathbb{R})}+\frac{1}{2}\left(h^{H_{K}^{\bullet}(X, \mathbb{R})}\right)^{-1} \nabla^{H_{K}^{\bullet}(X, \mathbb{R})} h^{H_{K}^{\bullet}(X, \mathbb{R})} .
$$

The connection $\nabla^{H_{K}^{*}(X, \mathbb{R}), u}$ induces on $\mathcal{H}_{K}^{\cdot}(X, \mathbb{C})$ the corresponding holomorphic Hermitian connection. Finally, under the canonical isomorphism $H_{K}^{\cdot}(X, \mathbb{R}) \simeq H^{\bullet}(Y, \mathbb{R})$, the connections $\nabla^{H_{K}^{\bullet}(X, \mathbb{R}), u}$ and $\nabla^{H^{*}(Y, \mathbb{R}), u}$ correspond.

Proof. The proof of the first part of our theorem is the same as the proof of Propositions 2.19 and 2.23 .

As we saw in Remark 2.24, the connection $\nabla^{H^{*}(Y, \mathbb{R}), u}$ is obtained by projecting the flat connection $\nabla^{H^{\bullet}(Y, \mathbb{R})}$ with respect to the Hodge splitting

$$
H^{\bullet}(Y, \mathbb{C})=\bigoplus_{0 \leqslant p, q \leqslant \ell} H^{(p, q)}(Y, \mathbb{C})
$$

Now we use (2.40) again and observe that the operator $w^{i} i_{T\left(\bar{f}_{\alpha}, w_{i}\right)}$ decreases the total degree by 2 with respect to the grading associated to $\mathcal{N}$. Therefore one can say that the connection $\nabla^{H^{\bullet}(Y, \mathbb{R}), u}$ is obtained from $\nabla^{H^{\bullet}(Y, R)}$ by projection with respect to the splitting associated to this new number operator. The same considerations apply to the connection $\nabla^{H_{K}^{*}(X, \mathbb{R})}$. Since the flat connections $\nabla^{H_{K}^{*}(X, \mathbb{R})}$ and $\nabla^{H^{*}(Y, \mathbb{R})}$ correspond, this shows that the unitary connections also correspond. The proof of our theorem is completed.

\section{The analytic torsion forms of the double complex}

In this section, we give an essentially local formula for the holomorphic torsion forms associated to the family of double complexes considered in $\S 5$, which extends the vanishing result of $\S 4$.

This section is organized as follows. In $\S 6.1$, we recall the definition of the $R(\theta, x)$ genus of [B94]. In $\S 6.2$, we define the superconnection forms of the double complexes. In $\S 6.3$, we define the associated holomorphic torsion forms $T_{K, g}\left(\omega^{M}\right)$. In $\S 6.4$, we consider the case where the fibrewise spectral sequence associated to the filtration $G$ of $\S 5.2$ degenerates. Then we give a formula for $T_{K, g}\left(\omega^{M}\right)$ in terms of Bott-Chern classes. In $\S 6.5$, we consider the case where $K=f^{X}$, with $f \in \mathfrak{g}$. In this case, we use the embedding formulas of [BL91, B97, BM02] to give a formula for $T_{K, g}\left(\omega^{M}\right)$ in terms of Bott-Chern classes and of a local formula involving the $R(\theta, x)$ genus of [B94]. In particular, we obtain a local formula for the Ray-Singer analytic holomorphic torsion of the fibres. Finally, in $\S 6.7$, we relate the superconnection forms of the double complexes to de Rham superconnection forms. 


\subsection{The genus $R(\theta, x)$}

Let

$$
L(\theta, s)=\sum_{n=1}^{+\infty} \frac{e^{i n \theta}}{n^{s}}
$$

be the Lerch series. Let $\zeta(\theta, s)$ and $\eta(\theta, s)$ be its real and imaginary parts, so that

$$
\zeta(\theta, s)=\sum_{n=1}^{+\infty} \frac{\cos (n \theta)}{n^{s}}, \quad \eta(\theta, s)=\sum_{n=1}^{+\infty} \frac{\sin (n \theta)}{n^{s}}
$$

Definition 6.1. For $\theta \in \mathbb{R} / 2 \pi \mathbb{Z}$, let $R(\theta, x)$ be the formal power series

$$
R(\theta, x)=\sum_{\substack{n \geqslant 0 \\ n \text { odd }}}\left\{\sum_{j=1}^{n} \frac{1}{j} \zeta(\theta,-n)+2 \frac{\partial \zeta}{\partial s}(\theta,-n)\right\} \frac{x^{n}}{n !}+\sum_{\substack{n \geqslant 0 \\ n \text { even }}} i\left\{\sum_{j=1}^{n} \frac{1}{j} \eta(\theta,-n)+2 \frac{\partial \eta}{\partial s}(\theta,-n)\right\} \frac{x^{n}}{n !} .
$$

Note that $R(0, x)$ is exactly the series $R(x)$ introduced by Gillet and Soulé [GS91]. In the sequel, given $\theta, R(\theta, x)$ will be identified to the corresponding additive genus.

Let $E$ be a complex Hermitian vector bundle equipped with a unitary automorphism $g$. We assume that $E$ splits as

$$
E=\bigoplus_{\theta \in \mathbb{R} / 2 \pi \mathbb{Z}} E^{\theta}
$$

so that $g$ acts on $E^{\theta}$ by multiplication by $e^{i \theta}$. Set

$$
R_{g}(E)=\sum_{\theta \in \mathbb{R} / 2 \pi \mathbb{Z}} R\left(\theta, E^{\theta}\right)
$$

\subsection{The holomorphic superconnection forms of the double complex}

We make the same assumptions as in $\S 5$.10. In particular $K$ is just assumed to be a $G$-invariant holomorphic section of $T X$.

Definition 6.2. For $t>0$, set

$$
\begin{gathered}
\alpha_{K, t}=\Phi \operatorname{Tr}_{\mathrm{s}}\left[g \exp \left(-B_{K, t}^{2}\right)\right], \quad \beta_{K, t}=\frac{1}{\sqrt{2 i \pi}} \Phi \operatorname{Tr}_{\mathrm{s}}\left[g\left(\frac{\partial}{\partial t} B_{K, t}\right) \exp \left(-B_{K, t}^{2}\right)\right], \\
\gamma_{K, t}=\Phi \operatorname{Tr}_{\mathrm{s}}\left[g \mathcal{N}_{t} \exp \left(-B_{K, t}^{2}\right)\right] .
\end{gathered}
$$

The same arguments as in Theorem 4.2 show that the analogue of this theorem still holds. In particular as $t \rightarrow 0$,

$$
\alpha_{K, t}=L(g)+\mathcal{O}(t), \quad \gamma_{K, t}=\frac{C_{K,-1}}{t}+C_{K, 0}+\mathcal{O}(t) .
$$

Using (4.19) and (4.66), instead of (4.14), we now have

$$
\begin{gathered}
C_{K,-1}=\int_{X_{g}} \frac{\omega^{M}}{2 \pi} c_{\max }\left(T X_{g}, h^{T X_{g}}\right), \\
C_{K, 0}=\frac{n}{2} L(g)-2 \int_{X_{g}} \operatorname{Td}_{g}^{\prime}\left(T X, h^{T X}\right) L_{g}\left(T X, h^{T X}\right)+\frac{\bar{\partial} \partial}{2 i \pi} D_{K, 0} .
\end{gathered}
$$




\section{J.-M. Bismut}

As $t \rightarrow+\infty$, Equation (4.15) should be replaced by

$$
\begin{gathered}
\alpha_{K, t}=\operatorname{ch}_{g}\left(\mathcal{H}_{K}^{\cdot}(X, \mathbb{C}), h^{\mathcal{H}_{K}^{\cdot}(X, \mathbb{C})}\right)+\mathcal{O}(1 / \sqrt{t}), \\
\gamma_{K, t}=\Phi \operatorname{Tr}_{\mathrm{S}}\left[\mathcal{N} \exp \left(-\nabla^{\mathcal{H}_{K}^{\bullet}(X, \mathbb{C}) u, 2}\right)\right]+\mathcal{O}(1 / \sqrt{t}) .
\end{gathered}
$$

Also the analogues of (4.16) and (4.17) still hold.

\subsection{The analytic torsion forms of the double complex}

Take $g \in G$. By using the results of $\S 6.2$, we can proceed as in $\S 4.3$ and construct analytic torsion forms $T_{K, g}\left(\omega^{M}\right)$ as in Definition 4.4. This form is a smooth form on $S$, which lies in $P^{S}$.

Proposition 6.3. The following identity holds:

$$
\frac{\bar{\partial} \partial}{2 i \pi} T_{K, g}\left(\omega^{M}\right)=\operatorname{ch}_{g}\left(\mathcal{H}_{K}^{\cdot}(X, \mathbb{C}), h^{\mathcal{H}} \cdot(X, \mathbb{C})\right)-L(g) .
$$

Proof. Our result follows [BGS88b, Theorem 2.20] [BK92, Theorem 3.9], and also follows from the analogue of (4.17) and from (6.7) and (6.9).

\subsection{The case where the spectral sequence degenerates}

In the present section, we will assume that the spectral sequence associated to the filtration $G$ degenerates at $E_{1} \simeq H^{\bullet}(X, \mathbb{C})$. As we saw in Remark 5.2, this is the case if the fibres $X$ are connected, and the vanishing locus of $K$ is fibrewise non-empty.

By (5.11),

$$
\operatorname{Gr} \mathcal{H}_{K}^{\cdot}(X, \mathbb{C})=H^{\bullet}(X, \mathbb{C}) .
$$

By a construction given in [BGS88a, $\S 1 \mathrm{f}]$, there is a well-defined Bott-Chern class

$$
\widetilde{\operatorname{ch}}_{g}\left(H^{\bullet}(X, \mathbb{C}), h^{H^{\bullet}(X, \mathbb{C})}, \mathcal{H}_{K}^{\cdot}(X, \mathbb{C}), h^{\mathcal{H}_{K}^{\bullet}(X, \mathbb{C})}\right) \in P^{S} / P^{S, 0},
$$

such that

$$
\begin{aligned}
\frac{\bar{\partial} \partial}{2 i \pi} \widetilde{\operatorname{ch}}_{g}\left(H^{\bullet}(X, \mathbb{C}), h^{H^{\bullet}(X, \mathbb{C})}, \mathcal{H}_{K}^{\cdot}(X, \mathbb{C}), h^{\mathcal{H}_{K}^{\bullet}(X, \mathbb{C})}\right) \\
\quad=\operatorname{ch}_{g}\left(\mathcal{H}_{K}^{\cdot}(X, \mathbb{C}), h^{\mathcal{H}_{K}^{\bullet}(X, \mathbb{C})}\right)-\operatorname{ch}_{g}\left(H^{\bullet}(X, \mathbb{C}), h^{H^{\bullet}(X, \mathbb{C})}\right) .
\end{aligned}
$$

Taking (4.23) into account, we can rewrite (6.12) in the form

$$
\frac{\bar{\partial} \partial}{2 i \pi} \widetilde{\operatorname{ch}}_{g}\left(H^{\bullet}(X, \mathbb{C}), h^{H^{\bullet}(X, \mathbb{C})}, \mathcal{H}_{K}^{\cdot}(X, \mathbb{C}), h^{\mathcal{H}_{K}^{\bullet}(X, \mathbb{C})}\right)=\operatorname{ch}_{g}\left(\mathcal{H}_{K}^{\cdot}(X, \mathbb{C}), h^{\mathcal{H}_{K}^{\bullet}(X, \mathbb{C})}\right)-L(g) .
$$

THEOREM 6.4. The following identity holds:

$$
T_{K, g}\left(\omega^{M}\right)=\widetilde{\operatorname{ch}}_{g}\left(H^{\bullet}(X, \mathbb{C}), h^{H^{\bullet}(X, \mathbb{C})}, \mathcal{H}_{K}^{\bullet}(X, \mathbb{C}), h^{\mathcal{H}_{K}^{\bullet}(X, \mathbb{C})}\right) \quad \text { in } P^{S} / P^{S, 0} .
$$

In particular $T_{K, g}(\omega) \in P^{S} / P^{S, 0}$ only depends on the class of $g$ in $G / G_{O}$.

Proof. Under the given assumptions, this is a direct consequence of results in [B97, Theorem 0.2] and [BM02, Theorem 0.2], and also of the vanishing result of Theorem 4.15. Since $G_{O}$ acts trivially on $H^{\bullet}(Y, \mathbb{R})$, by $(5.10), G_{O}$ acts trivially on $\mathcal{H}_{K}^{\cdot}(X, \mathbb{C})$. Therefore the right-hand side of $(6.14)$ depends only on the class of $g$ in $G / G_{O}$. 


\section{HOLOMORPHIC AND DE RHAM TORSION}

Remark 6.5. Using in particular (6.11), it is clear that $T_{z K, g}\left(\omega^{M}\right)$ depends continuously on $z \in \mathbb{C}$, the point being that the continuous dependence also extends to $z=0$. This is also clear for the right-hand side of (6.14).

Using Theorem 5.16 and proceeding as in [BLo95, Proposition 1.3], we find that if there is a $G$-invariant $f \in \mathfrak{g}$ such that $K=f^{X}$, then

$$
\operatorname{ch}_{g}\left(H_{K}^{\cdot}(X, \mathbb{R}), h^{\mathcal{H}_{K}^{\cdot}(X, \mathbb{R})}\right)=L(g) .
$$

By (6.13) and (6.15),

$$
\frac{\bar{\partial} \partial}{2 i \pi} \widetilde{c h}_{g}\left(H^{\bullet}(X, \mathbb{C}), h^{H^{\bullet}(X, \mathbb{C})}, \mathcal{H}_{K}^{\cdot}(X, \mathbb{C}), h^{\mathcal{H}_{K}^{\cdot}(X, \mathbb{C})}\right)=0
$$

\subsection{An evaluation of $T_{K, g}\left(\omega^{M}\right)$ when $K=f^{X}$}

Assume that there is $f \in \mathfrak{g}$ which is $G$-invariant and such that $K=f^{X}$. Let $N$ be the vanishing locus of $K$. Then $N$ fibres on $S$, with fibre $Y$.

Recall that since $\mathcal{H}_{K}^{\cdot}(X, \mathbb{C}) \simeq H^{\bullet}(Y, \mathbb{C})$, the metrics $h^{\mathcal{H}_{K}^{\bullet}(X, \mathbb{C})}$ and $h^{H^{\bullet}(Y, \mathbb{C})}$ can both be viewed as metrics on $H^{\bullet}(Y, \mathbb{C})$. By $(4.23)$ and $(6.15)$, the Bott-Chern class

$$
\widetilde{\operatorname{ch}}_{g}\left(H^{\bullet}(Y, \mathbb{C}), h^{\mathcal{H}_{K}^{\bullet}(X, \mathbb{C})}, h^{H^{\bullet}(Y, \mathbb{C})}\right) \in P^{S} / P^{S, 0}
$$

constructed in [BGS88a, $\S 1 \mathrm{f}$ ], is such that

$$
\begin{aligned}
\frac{\bar{\partial} \partial}{2 i \pi} \widetilde{c h}_{g}\left(H^{\bullet}(Y, \mathbb{C}), h^{\mathcal{H}_{K}(X, \mathbb{C})}, h^{H^{\bullet}(Y, \mathbb{C})}\right) \\
\quad=\operatorname{ch}_{g}\left(H^{\bullet}(Y, \mathbb{C}), h^{H^{\bullet}(Y, \mathbb{C})}\right)-\operatorname{ch}_{g}\left(H^{\bullet}(Y, \mathbb{C}), h^{\mathcal{H}_{K}(Y, \mathbb{C})}\right)=0 .
\end{aligned}
$$

Recall that $\nabla K$ induces an invertible endomorphism of $N_{Y / X}$. Then $\left.|\nabla K|_{N_{Y / X}}\right|^{2}$ is a self-adjoint positive endomorphism of $N_{Y / X}$.

Theorem 6.6. The following identity holds:

$$
\begin{aligned}
\widetilde{c h}_{g}( & \left.H^{\bullet}(Y, \mathbb{C}), h^{\mathcal{H}}{ }_{K}^{(X, \mathbb{C})}, h^{H^{\bullet}(Y, \mathbb{C})}\right)+T_{K, g}^{M}\left(\omega^{M}\right) \\
& =\int_{Y_{g}} c_{\max }\left(T Y_{g}\right)\left(R_{g}\left(N_{Y / X}\right)-\left\langle\left(\mathrm{Td}^{\prime} / \mathrm{Td}\right)_{g}\left(N_{Y / X}\right),\left.\log |\nabla K|_{N_{Y / X}}\right|^{2}\right\rangle\right) \quad \text { in } P^{S} / P^{S, 0} .
\end{aligned}
$$

Proof. The idea of the proof is to use [B97, Theorem 0.1] and [BM02, Theorem 0.1].

Indeed suppose first that $N$ is a finite cover of $S$, i.e. the fibres $Y$ are a collection of points. Then the Koszul complex $\left(\Lambda^{\bullet}\left(T^{*} X\right), i_{K}\right)$ together with the restriction map $j^{*}$ provides us with a resolution of the sheaf $j_{*} \mathcal{O}_{N}$, i.e. we have the exact sequence of sheaves on $M$,

$$
0 \rightarrow \mathcal{O}_{M}\left(\Lambda^{\ell}\left(T^{*} X\right)\right) \stackrel{i_{K}}{\rightarrow} \cdots \mathcal{O}_{M}\left(\Lambda^{\ell-1}\left(T^{*} X\right)\right) \cdots \stackrel{i_{K}}{\rightarrow} \mathcal{O}_{M}(\mathbb{C}) \stackrel{j^{*}}{\rightarrow} j_{*} \mathcal{O}_{N}(\mathbb{C}) \rightarrow 0 .
$$

We can then directly use the results of [B97] and [BM02]. We briefly explain how to adapt these results in the situation which is considered here. By Theorem 4.15,

$$
T_{g}\left(j^{*} \omega^{M}\right)=0 \quad \text { in } H^{\bullet}(S, \mathbb{C}) .
$$

Observe that since $K$ is $G$-invariant, $\left.K\right|_{M_{g}}$ is a section of $T X_{g}$. Let $j_{g}: N_{g} \rightarrow M_{g}$ be the obvious embedding. Then the Koszul complex $\left(\Lambda^{*}\left(T^{*} X_{g}\right), i_{K}\right)$ is a resolution of the sheaf $j_{g}^{*} \mathcal{O}_{N_{g}}(\mathbb{C})$. Tautologically, it verifies assumption $(A)$ of [B90b] with respect to a $G$-invariant metric on $N_{Y / X}$. Note that this metric is in general distinct from the metric one obtains by identifying $N_{Y / X}$ to the orthogonal vector bundle to $T Y$ in $\left(\left.T X\right|_{N}, h^{\left.T X\right|_{N}}\right)$. However the anomaly formulas of [BK92] and 


\section{J.-M. Bismut}

[M00] on the analytic torsion forms and their equivariant version show that one can adapt without any change the results of [B97] and [BM02].

We now describe these results in the specific situation which is considered here. By a construction given in [BGS90, Theorem 3.14], to this Koszul complex and to the metric $h^{T X_{g}}$, one can associate a canonically defined current $\widetilde{c}_{\max }\left(T X_{g}, h^{T X_{g}}\right)$ on $M_{g}$, of degree $\left(\ell_{g}-1, \ell_{g}-1\right)$, such that

$$
\frac{\bar{\partial} \partial}{2 i \pi} \widetilde{c}_{\max }\left(T X_{g}, h^{T X_{g}}\right)=\delta_{N_{g}}-c_{\max }\left(T X_{g}, h^{T X_{g}}\right) .
$$

Set

$$
\widetilde{T}\left(T X_{g}, h^{T X_{g}}\right)=\operatorname{Td}^{-1}\left(T X_{g}, h^{T X_{g}}\right) \widetilde{c}_{\max }\left(T X_{g}, h^{T X_{g}}\right) .
$$

By (6.21) and (6.22), we get

$$
\frac{\bar{\partial} \partial}{2 i \pi} \widetilde{T}\left(T X_{g}, h^{T X_{g}}\right)=\operatorname{Td}^{-1}\left(T X_{g}, h^{T X_{g}}\right) \delta_{N_{g}}-\operatorname{ch}\left(\Lambda^{\bullet}\left(T^{*} X_{g}\right), h^{\Lambda^{\bullet}\left(T^{*} X_{g}\right)}\right) .
$$

By [BGS90, Theorem 3.17], the Bott-Chern current $\widetilde{T}\left(T X_{g}, h^{T X_{g}}\right)$ is exactly the current (evaluated modulo irrelevant $\partial$ or $\bar{\partial}$ coboundaries) one can associate to the above resolution on $M_{g}$.

On the other hand, on $M_{g}$, we have the holomorphic and metric splitting

$$
\left.T X\right|_{M_{g}}=T X_{g} \oplus N_{X_{g} / X}
$$

It is then elementary to verify that the current $\mathfrak{T}_{g}\left(T X, h^{T X}\right)$ on $M_{g}$ which is constructed in [B95, $\S \mathrm{VI}]$ is given by

$$
\mathfrak{T}_{g}\left(T X, h^{T X}\right)=\operatorname{ch}_{g}\left(\Lambda^{\bullet}\left(N_{X_{g} / X}^{*}\right), h^{\Lambda^{\bullet}\left(N_{X_{g} / X}^{*}\right)}\right) \widetilde{T}\left(T X_{g}, h^{T X_{g}}\right) .
$$

In particular, by (6.22) and (6.23), we get

$$
\frac{\bar{\partial} \partial}{2 i \pi} \mathfrak{T}_{g}\left(T X, h^{T X}\right)=\operatorname{Td}_{g}^{-1}\left(T X, h^{T X}\right) \delta_{N_{g}}-\operatorname{ch}_{g}\left(\Lambda^{\bullet}\left(T^{*} X\right), h^{\Lambda^{\bullet}\left(T^{*} X\right)}\right) .
$$

By (6.22) and (6.25), we get

$$
\operatorname{Td}_{g}\left(T X, h^{T X}\right) \mathfrak{T}_{g}\left(T X, h^{T X}\right)=\widetilde{c}_{\max }\left(T X_{g}, h^{T X_{g}}\right) .
$$

In particular the above current has degree $\left(\ell_{g}-1, \ell_{g}-1\right)$. So its integral along the fibre $X_{g}$ vanishes identically. An application of the formula of [B97] and [BM02] then leads to (6.18).

Now we explain how to obtain (6.18) in the general case. Strictly speaking, we cannot use directly the above results, because the complex $\left(\Lambda^{\bullet}\left(T^{*} X\right), i_{K}\right)$ does not give a projective resolution of a sheaf localized on $N$. However as we saw in the proof of Theorem 5.1, the map $j^{*}$ gives a quasiisomorphism of the complex of $\mathbb{Z}$-graded sheaves $\left(\Lambda^{*}\left(T^{*} X\right), i_{K}\right)$ and $\left(\Lambda^{*}\left(T^{*} Y\right), 0\right)$, the $\mathbb{Z}$-grading being defined by $\mathcal{N}$. From this, we showed in Theorem 5.1 that $j^{*}:\left(\Omega^{\bullet}(X, \mathbb{R}), \bar{\partial}_{K}^{X}\right) \rightarrow\left(\Omega^{\bullet}(Y), \bar{\partial}^{Y}\right)$ is a quasi-isomorphism. We then claim that all the arguments in the above references can be adapted without any change. Indeed the general scheme of the proof remains the same. All the local index theoretic computations are also strictly identical except for assumption $(A)$ of $[\mathrm{B} 90 \mathrm{~b}]$ and for the behaviour as a parameter $T \rightarrow+\infty$ of the fibrewise harmonic forms, which was obtained in [BL91, ch. 10] and in [B97, ch. 10].

We will explain in some detail how to get around these difficulties. First it will be convenient to equip the twisting vector bundle $\Lambda^{\bullet}\left(T^{*} X\right)$ with a $G$-invariant metric coming from a new $G$-invariant metric $\widetilde{h}^{T X}$. Let $h^{N_{Y / X}}$ be the metric on $N_{Y / X}$ which is obtained by identifying $N_{Y / X}$ to the orthogonal bundle to $T Y$ in $\left.T X\right|_{N}$. We just require that the metric induced by $\widetilde{h}^{T X}$ on $\left.\operatorname{Im}\left(\left.\nabla^{T X} K\right|_{N}\right) \subset T X\right|_{N}$ is the image of $h^{N_{Y / X}}$ by the map $f \rightarrow \nabla_{f}^{T X} K$, and also that it induces on $T Y$ the given metric $h^{T Y}$. This is the analogue of assumption $(A)$ in the present situation. 


\section{HOLOMORPHIC AND DE RHAM TORSION}

Then one verifies easily that as far as local index theoretic computations are concerned, the situation is exactly the same as in [B97]. The only remaining difficulty is the behaviour of the eigenvalues of the operator $\left(\bar{\partial}_{T K}^{X}+\bar{\partial}_{T K}^{X *}\right)^{2}$ as $T \rightarrow+\infty$. Because of the quasi-isomorphism of Theorem 5.1, there are no 'small' eigenvalues which appear in the process.

The Bott-Chern currents which appear in the process are exactly the ones obtained in [B92]. Indeed in [B92, Theorem 3.6], a current $\widetilde{c}_{\max }\left(T X_{g}, h^{T X_{g}}\right)$ was obtained on $X_{g}$ such that

$$
\frac{\bar{\partial} \partial}{2 i \pi} \widetilde{c}_{\max }\left(T X_{g}, h^{T X_{g}}\right)=c_{\max }\left(T Y_{g}, h^{T Y_{g}}\right) \delta_{N_{g}}-c_{\max }\left(T X_{g}, h^{T X_{g}}\right) .
$$

We still define $\widetilde{T}\left(T X_{g}, h^{T X_{g}}\right)$ as in (6.22). Ultimately, it is still the integral along the fibre of $\widetilde{c}_{\max }\left(T Y, h^{T Y}\right)$ which appears in the formula. This integral vanishes identically. So we still obtain Equation (6.18). The proof of our theorem is completed.

Now we will specialize Theorem 6.6 in degree 0.

THEOREM 6.7. The following identity holds:

$$
T_{K, g}^{M}\left(\omega^{M}\right)^{(0)}=\int_{Y_{g}} c_{\max }\left(T Y_{g}\right)\left(R_{g}\left(N_{Y / X}\right)-\left\langle\left(\mathrm{Td}^{\prime} / \mathrm{Td}\right)_{g}\left(N_{Y / X}\right)-\frac{1}{2},\left.\log |\nabla K|_{N_{Y / X}}\right|^{2}\right\rangle\right)^{(0)} .
$$

Proof. Clearly,

$$
\widetilde{\operatorname{ch}}_{g}\left(H^{\bullet}(Y, \mathbb{C}), h^{\mathcal{H}_{K}^{\cdot}(X, \mathbb{C})}, h^{H^{\bullet}(Y, \mathbb{C})}\right)^{(0)}=\log \left(\frac{\|_{\lambda_{K}^{X}}}{\|_{\lambda^{Y}}}\right)^{2}(g) .
$$

By Theorems 5.8 and 6.6, we get (6.29).

Remark 6.8. Assume that the fibres $X$ are connected, and the fibres $Y$ are non-empty. As we saw in Remark 6.5, $T_{z K, g}\left(\omega^{M}\right)$ depends continuously on $z \in \mathbb{C}$. We will show in $\S 7$ how to reconcile this fact with Theorem 6.7 .

Now we assume that the fibres $X$ are connected and the fibres $Y$ are non-empty, so that the spectral sequence associated to the filtration $G$ degenerates at $E_{1}$. Let $\widetilde{c h}\left(H^{\bullet}(X, \mathbb{C}), h^{H^{\bullet}(X, \mathbb{C})}, H^{\bullet}(Y, \mathbb{C})\right.$, $\left.h^{H^{\bullet}(Y, \mathbb{C})}\right) \in P^{S} / P^{S, 0}$ be the associated Bott-Chern class as in [BGS88a, $\left.\S 1 \mathrm{f}\right]$. Then

$$
\frac{\bar{\partial} \partial}{2 i \pi} \widetilde{c h}_{g}\left(H^{\bullet}(X, \mathbb{C}), h^{H^{\bullet}(X, \mathbb{C})}, H^{\bullet}(Y, \mathbb{C}), h^{H^{\bullet}(Y, \mathbb{C})}\right)=0 .
$$

TheOrem 6.9. The following identity holds:

$$
\begin{aligned}
\widetilde{c h}_{g}\left(H^{\bullet}(X, \mathbb{C}), h^{H^{\bullet}(X, \mathbb{C})}, H^{\bullet}(Y, \mathbb{C}), h^{H^{\bullet}(Y, \mathbb{C})}\right) \\
\quad=\int_{Y_{g}} c_{\max }\left(T Y_{g}\right)\left(R_{g}\left(N_{Y / X}\right)-\left\langle\left(\mathrm{Td}^{\prime} / \mathrm{Td}\right)_{g}\left(N_{Y / X}\right),\left.\log |\nabla K|_{N_{Y / X}}\right|^{2}\right\rangle\right) \quad \text { in } P^{S} / P^{S, 0} .
\end{aligned}
$$

In particular the class of (6.32) in $P^{S} / P^{S, 0}$ depends only on the class of $g$ in $G / G_{o}$.

Proof. Note that by the functoriality of Bott-Chern classes [BGS88a, $\S 1 \mathrm{f}$ ],

$$
\begin{gathered}
\widetilde{\operatorname{ch}}_{g}\left(H^{\bullet}(X, \mathbb{C}), h^{H^{\bullet}(X, \mathbb{C})}, \mathcal{H}_{K}^{\cdot}(X, \mathbb{C}), h^{\mathcal{H}} \mathcal{H}_{K}(X, \mathbb{C})+\widetilde{\operatorname{ch}}_{g}\left(H^{\bullet}(Y, \mathbb{C}), h^{\mathcal{H}_{K}^{\bullet}(X, \mathbb{C})}, h^{H^{\bullet}(Y, \mathbb{C})}\right)\right. \\
=\widetilde{\operatorname{ch}}_{g}\left(H^{\bullet}(X, \mathbb{C}), h^{H^{\bullet}(X, \mathbb{C})}, H^{\bullet}(Y, \mathbb{C}), h^{H^{\bullet}(Y, \mathbb{C})}\right) .
\end{gathered}
$$

Our theorem then follows from Theorems 6.4 and 6.6 and from (6.33). 


\section{J.-M. Bismut}

\subsection{The de Rham superconnection forms}

We make the same assumptions as in $\S 6.5$. In particular, we still assume that $K=f^{X}$.

DeFinition 6.10. For $t>0$, set

$$
a_{K, t}=\Phi \operatorname{Tr}_{\mathrm{s}}\left[g \exp \left(-C_{\gamma_{K}, t}^{2}\right)\right], \quad b_{K, t}=\frac{1}{\sqrt{2 i \pi}} \Phi \operatorname{Tr}_{\mathrm{s}}\left[g \frac{\partial}{\partial t} C_{\gamma_{K}, t} \exp \left(-C_{\gamma_{K}, t}^{2}\right)\right] .
$$

Proposition 6.11. The following identities hold:

$$
a_{K, t}=L(g), \quad b_{K, t}=0 .
$$

Proof. The proof of our proposition is the same as the proof of Proposition 4.7.

\subsection{Comparing the holomorphic and de Rham superconnection forms}

We make the same assumptions as in $\S 6.6$.

Theorem 6.12. For any $t>0$, the following identities hold:

$$
\alpha_{K, t}=L(g), \quad \beta_{K, t}=0, \quad d \gamma_{K, t}=0 .
$$

Moreover,

$$
\gamma_{K, t}=\Phi \operatorname{Tr}_{\mathrm{s}}\left[g \mathcal{N}_{t} \exp \left(-C_{\gamma_{K}, 2 t}^{2}\right)\right] .
$$

The forms $\Phi^{-1} \gamma_{K, t}$ are purely imaginary.

Proof. Using Theorem 5.14 and Proposition 6.11 instead of Theorem 3.5 and Proposition 4.7, the proof of (6.36) and (6.37) is the same as the proof of Theorem 4.8. To prove that $\gamma_{K, t}$ is purely imaginary, we proceed as in the proof of Theorem 4.8. Namely we use the fact that $N^{(1,0)}$ and $N^{(0,1)}$ are conjugate operators, while $g$ and $\exp \left(-C_{\gamma_{K}, 2 t}^{2}\right)$ are real operators. From (6.37), we deduce that $\Phi^{-1} \gamma_{K, t}$ is purely imaginary.

Theorem 6.13. The form $T_{K, g}\left(\omega^{M}\right) \in P^{S}$ is closed. Moreover $\Phi^{-1} T_{K, g}\left(\omega^{M}\right)$ is purely imaginary.

Proof. By Theorem 6.12, the forms $\gamma_{K, t}$ are closed, and their image by $\Phi^{-1}$ is purely imaginary, which establishes our theorem.

Remark 6.14. By Theorem 6.4, we know that the class of $T_{K, g}\left(\omega^{M}\right)$ in $P^{S} / P^{S, 0}$ depends only on the class of $g$ in $G / G_{O}$. In Theorem 7.7, we will show that $T_{K, g}\left(\omega^{M}\right)^{(0)}$ vanishes.

\section{Rigidity, the genus $R(\theta, x)$ in degree 0 and the vanishing of $T_{K, g}\left(\omega^{M}\right)^{(0)}$}

The purpose of this section is to establish rigidity properties of genera which are related to the function $R(\theta, 0)$. Also we will show that $T_{K, g}\left(\omega^{M}\right)^{(0)}$ vanishes.

This section is organized as follows. In $\S 7.1$, we introduce the rigid genus associated to the obvious rigid action of $G$ on the $H^{(p, \cdot)}(X, \mathbb{C})$. If $f \in \mathfrak{g}$ is $G$-invariant, and if $K=f^{X}$, by taking a Mellin transform of a constant genus, we obtain a vanishing genus depending on $s \in \mathbb{C}$. In $\S 7.2$, by taking the derivative of this identity at $s=0$, we get a non-trivial identity involving the function $R(\theta, 0)$, and we show that this identity is equivalent to the vanishing of $T_{K, g}\left(\omega^{M}\right)^{(0)}$.

\subsection{A rigid genus}

We make the same assumptions as in $\S 6.5$. Take $g \in G$. 


\section{HOLOMORPHIC AND DE RHAM TORSION}

Proposition 7.1. The following identity holds:

$$
\sum_{p=0}^{\operatorname{dim} X}(-1)^{p} p \operatorname{ch}_{g}\left(H^{(p, \cdot)}(X, \mathbb{C})\right)=\int_{X_{g}} c_{\max }\left(T X_{g}\right)\left(\mathrm{Td}^{\prime} / \mathrm{Td}\right)_{g}(T X) \text { in } H^{\bullet}(S, \mathbb{C}) .
$$

Proof. Observe that

$$
\operatorname{Td}_{g}(T X) L_{g}(T X)=c_{\max }\left(T X_{g}\right) .
$$

Our proposition follows from (4.19) in Theorem 4.2, from the differentiation of (4.67) at $b=0$ and from (7.2).

Take now $f \in \mathfrak{g}$ which is $g$-invariant. Set $K=f^{X}$. Then $X_{g}$ and $Y$ intersect along $Y_{g}$. Moreover we have a holomorphic splitting,

$$
N_{Y_{g} / X}=N_{Y_{g} / X_{g}} \oplus N_{Y_{g} / Y} \oplus \tilde{N} .
$$

The definition of $\tilde{N}$ is self-explanatory. Each vector bundle on the right-hand side of (7.3) is stable by $g$ and by $\left.\nabla K\right|_{Y_{g}}$.

In particular $\left.\nabla K\right|_{Y_{g}}$ acts on $N_{Y / X}=N_{Y_{g} / X_{g}} \oplus \tilde{N}$ with locally constant eigenvalues $i \alpha, \alpha \in \mathbb{R}^{*}$. Let $N_{Y / X}^{ \pm}$be the subbundle of $N_{Y / X}$ corresponding to $\alpha>0$ or $\alpha<0$. Then

$$
N_{Y / X}=N_{Y / X}^{+} \oplus N_{Y / X}^{-} \cdot
$$

Recall that $N$ is the vanishing locus of $K$. Let $N_{g} \subset N$ be the fixed point set of $g$. Then $N_{g}$ fibres on $S$ with fibre $Y_{g}$. For any $t \in \mathbb{R}^{*}$, on $N_{g}$, the cohomology classes $\left(\mathrm{Td}^{\prime} / \mathrm{Td}\right)_{g}(T Y)$ and $\left(\mathrm{Td}^{\prime} / \mathrm{Td}\right)_{g e^{i t K}}\left(N_{Y / X}\right)$ are well defined.

Theorem 7.2. For any $t \in \mathbb{R}^{*}$, the following identity holds:

$$
\begin{aligned}
& \sum_{p=0}^{\operatorname{dim} X}(-1)^{p} p \operatorname{ch}_{g}\left(H^{(p, \cdot)}(X, \mathbb{C})\right) \\
& \quad=\int_{Y_{g}} c_{\max }\left(T Y_{g}\right)\left(\left(\mathrm{Td}^{\prime} / \mathrm{Td}\right)_{g}(T Y)+\left(\mathrm{Td}^{\prime} / \mathrm{Td}\right)_{g e^{i t K}}\left(N_{Y / X}\right)\right) \text { in } H^{\bullet}(S, \mathbb{C}) .
\end{aligned}
$$

In particular,

$$
\sum_{p=0}^{\operatorname{dim} X}(-1)^{p} p \operatorname{ch}_{g}\left(H^{(p, \cdot)}(X, \mathbb{C})\right)=\int_{Y_{g}} c_{\max }\left(T Y_{g}\right)\left(\left(\operatorname{Td}^{\prime} / \mathrm{Td}\right)_{g}(T Y)+\operatorname{dim} N_{Y / X}^{ \pm}\right) \quad \text { in } H^{\bullet}(S, \mathbb{C}) .
$$

Proof. Take $z \in \mathbb{R}^{*}$. Then for $z$ close enough to $0, X_{g e^{z K}}=Y_{g}$. By Proposition 7.1, for $z \in \mathbb{R}^{*}$ close enough to 0 ,

$$
\sum_{p=0}^{\operatorname{dim} X}(-1)^{p} p \operatorname{ch}_{g e^{z K}}\left(H^{(p, \cdot)}(X, \mathbb{C})\right)=\int_{Y_{g}} c_{\max }\left(T Y_{g}\right)\left(\mathrm{Td}^{\prime} / \mathrm{Td}\right)_{g e^{z K}}(T X) \text { in } H^{\bullet}(S, \mathbb{C}) .
$$

Moreover $G_{O}$ acts trivially on $H^{\bullet}(X, \mathbb{C})$, so that on the left-hand side of $(7.7)$, we can as well replace $g e^{z K}$ by $g$. The right-hand side of (7.7) extends tautologically to a holomorphic function of $z$, which turns out to be constant. We can then change $z$ into it, with $t \in \mathbb{R}^{*}$, and we get (7.5) by using the fact that $\mathrm{Td}^{\prime} / \mathrm{Td}$ is additive. When making $t \rightarrow \pm \infty$ in (7.5), we get (7.6) easily.

Remark 7.3. Using (7.7) and the fact that the limit of the right-hand side exists when $z=i t$ and $t \rightarrow \pm \infty$, one can recover the fact that (7.7) does not depend on $z$. 


\section{J.-M. Bismut}

As we saw before, the eigenvalues $i \alpha$ of $\left.\nabla K\right|_{Y}$ acting on $N_{Y / X}$ are locally constant. We can then split $N_{Y / X}$ into the eigenbundles $N_{Y / X}^{\alpha}$, so that

$$
N_{Y / X}=\bigoplus N_{Y / X}^{\alpha}
$$

The splitting (7.8) refines on (7.4). In the sequel, given $s \in \mathbb{C}$, we identify $\zeta(-\cdot, s), \eta(-i \cdot, s)$ to the corresponding additive genera. In particular since $g$ acts on the $N_{Y / X}^{\alpha}, \zeta(-i \cdot, s)_{g}\left(N_{Y / X}^{\alpha}\right)$, $\eta(-i \cdot, s)_{g}\left(N_{Y / X}^{\alpha}\right)$ are well-defined cohomology classes on $Y_{g}$. If $g$ acts on $N_{N_{Y / X}}^{\alpha}$ like $e^{i \theta}$, if the $x_{j}$ are the Chern roots of $N_{N_{Y / X}}^{\alpha}$, then

$$
\begin{aligned}
& \zeta(-i \cdot, s)_{g}\left(N_{Y / X}^{\alpha}\right)=\sum_{j} \zeta\left(-i\left(x_{j}+i \theta\right), s\right), \\
& \eta(-i \cdot, s)_{g}\left(N_{Y / X}^{\alpha}\right)=\sum_{j} \eta\left(-i\left(x_{j}+i \theta\right), s\right) .
\end{aligned}
$$

Theorem 7.4. For any $s \in \mathbb{C}$, the following identities hold:

$$
\begin{gathered}
\int_{Y_{g}} c_{\max }\left(T Y_{g}\right) \sum \operatorname{sgn} \alpha|\alpha|^{-s} \zeta(-i \cdot, s)_{g}\left(N_{Y / X}^{\alpha}\right)=0 \text { in } H^{\bullet}(S, \mathbb{C}), \\
\int_{Y_{g}} c_{\max }\left(T Y_{g}\right) \sum|\alpha|^{-s} \eta(-i \cdot, s)_{g}\left(N_{Y / X}^{\alpha}\right)=0 \text { in } H^{\bullet}(S, \mathbb{C}) .
\end{gathered}
$$

Moreover,

$$
\int_{Y_{g}} c_{\max }\left(T Y_{g}\right) \sum|\alpha|^{-s}\left(\frac{\partial}{\partial s} \eta(-i \cdot, s)_{g}\left(N_{Y / X}^{\alpha}\right)-\log (|\alpha|) \eta(-i \cdot, s)_{g}\left(N_{Y / X}^{\alpha}\right)\right)=0 \text { in } H^{\bullet}(S, \mathbb{C}) .
$$

In particular,

$$
\int_{Y_{g}} c_{\max }\left(T Y_{g}\right) \sum\left(\frac{\partial}{\partial s} \eta(-i \cdot, 0)_{g}\left(N_{Y / X}^{\alpha}\right)-\log (|\alpha|) \eta(-i \cdot, 0)_{g}\left(N_{Y / X}^{\alpha}\right)\right)=0 \text { in } H^{\bullet}(S, \mathbb{C}) .
$$

Proof. Consider the Mellin transform in the variable $t \in \mathbb{R}_{+}^{*}$ of the identity (7.5) in Theorem 7.2, while taking into account the fact that the right-hand side of (7.6) is the limit of (7.5) as $t \rightarrow \pm \infty$. This Mellin transform vanishes tautologically. Moreover, if $\alpha \in \mathbb{R}^{*}, x \in \mathbb{C}$,

$$
\frac{1}{\Gamma(s)} \int_{0}^{+\infty} \frac{-e^{-(x-t \alpha)}}{1-e^{-(x-t \alpha)}} t^{s-1} d t=\operatorname{sgn} \alpha|\alpha|^{-s} L(-\operatorname{sgn} \alpha i x, s) .
$$

Then we get from (7.5) and (7.13),

$$
\int_{Y_{g}} c_{\max }\left(T Y_{g}\right) \sum \operatorname{sgn} \alpha|\alpha|^{-s} L(-\operatorname{sgn} \alpha i \cdot, s)_{g}\left(N_{Y / X}^{\alpha}\right)=0 \text { in } H^{\bullet}(S, \mathbb{C}) .
$$

We can rewrite (7.14) in the form

$$
\int_{Y_{g}} c_{\max }\left(T Y_{g}\right) \sum|\alpha|^{-s}\left(\operatorname{sgn} \alpha \zeta(-i \cdot, s)_{g}\left(N_{Y / X}^{\alpha}\right)+i \eta(-i \cdot, s)_{g}\left(N_{Y / X}^{\alpha}\right)\right)=0 .
$$

Using (7.15) for $K$ and $-K$, we get (7.10). By differentiating the second equality in (7.10), we get (7.11), and (7.12) when making $s=0$. 


\section{HOLOMORPHIC AND DE RHAM TORSION}

Remark 7.5. Recall that

$$
\begin{aligned}
L(\theta, 0) & =\frac{e^{i \theta}}{1-e^{i \theta}} \quad \text { if } \theta \notin 2 \pi \mathbb{Z}, \\
& =-\frac{1}{2} \quad \text { if } \theta \in 2 \pi \mathbb{Z},
\end{aligned}
$$

so that

$$
\begin{aligned}
\zeta(\cdot, 0) & =-\frac{1}{2} \\
\eta(\theta, 0) & =\frac{1}{2} \cot (\theta / 2) \quad \text { if } \theta \notin 2 \pi \mathbb{Z}, \\
& =0 \quad \text { if } \theta \in 2 \pi \mathbb{Z} .
\end{aligned}
$$

From (7.10) and (7.17), we recover the fact that the right-hand side of (7.6) does not depend on the choice of sign. Also observe that when replacing $g$ by $g e^{z K}$ with $z \in \mathbb{R}$ close enough to 0 , by differentiating (7.10) in the variable $z$, we find that the two identities in (7.10) are equivalent to each other. Also observe that when replacing $K$ by $t K$ for $t \in \mathbb{R}^{*}$, we get a new identity (7.12), which is equivalent to the initial one because of the second identity in (7.10).

\subsection{The case where $S$ is a point}

THEOREM 7.6. The following identity holds:

$$
\int_{Y_{g}} c_{\max }\left(T Y_{g}\right)\left(R_{g}\left(N_{Y / X}\right)-\left\langle\left(\mathrm{Td}^{\prime} / \mathrm{Td}\right)_{g}\left(N_{Y / X}\right)-\frac{1}{2},\left.\log |\nabla K|_{N_{Y / X}}\right|^{2}\right\rangle\right)^{(0)}=0 .
$$

Proof. Recall that $\widetilde{N}$ was defined in (7.3). Then

$$
\left(\mathrm{Td}^{\prime} / \mathrm{Td}\right)_{g}\left(N_{Y / X}\right)=\left(\mathrm{Td}^{\prime} / \mathrm{Td}\right)\left(N_{Y_{g} / X_{g}}\right)+\left(\mathrm{Td}^{\prime} / \mathrm{Td}\right)_{g}(\tilde{N}) .
$$

Since

we find that

$$
\operatorname{Td}(x)=1+\frac{1}{2} x+\cdots,
$$

$$
\int_{Y_{g}} c_{\max }\left(T Y_{g}\right)\left\langle\left(\mathrm{Td}^{\prime} / \mathrm{Td}\right)\left(N_{Y_{g} / X_{g}}\right)-\frac{1}{2},\left.\log |\nabla K|_{N_{Y_{g} / X_{g}}}\right|^{2}\right\rangle=0 .
$$

Set

$$
\widehat{A}(x)=\frac{1}{2 \sinh (x / 2)} .
$$

Then

$$
\left(\widehat{A}^{\prime} / \widehat{A}\right)(x)=-\frac{1}{2} \operatorname{coth}(x / 2) .
$$

Moreover,

$$
\operatorname{Td}_{\theta}(x)=\widehat{A}(x+i \theta) e^{(x+i \theta) / 2} .
$$

From (7.24), we deduce that

$$
\left(\operatorname{Td}_{\theta}^{\prime} / \operatorname{Td}_{\theta}\right)(x)=\left(\widehat{A}^{\prime} / \widehat{A}\right)(x+i \theta)+\frac{1}{2} .
$$

By (7.17) and (7.23)-(7.25), we get

$$
\int_{Y_{g}} c_{\max }\left(T Y_{g}\right)\left\langle\left(\mathrm{Td}^{\prime} / \mathrm{Td}\right)_{g}(\tilde{N})-\frac{1}{2},\left.\log |\nabla K|_{\tilde{N}}\right|^{2}\right\rangle^{(0)}=\int_{Y_{g}} c_{\max }\left(T Y_{g}\right) \sum \log |\alpha| 2 i \eta(-i \cdot, 0)_{g}\left(\tilde{N}^{\alpha}\right)^{(0)} \text {. }
$$


Clearly, $\eta(0, s)=0$. Therefore, from (7.19), (7.21) and (7.26), we obtain

$$
\begin{gathered}
\int_{Y_{g}} c_{\max }\left(T Y_{g}\right)\left\langle\left(\mathrm{Td}^{\prime} / \mathrm{Td}\right)_{g}\left(N_{Y / X}\right)-\frac{1}{2},\left.\log |\nabla K|_{N_{Y / X}}\right|^{2}\right\rangle^{(0)} \\
=\int_{Y_{g}} c_{\max }\left(T Y_{g}\right) \sum \log |\alpha| 2 i \eta(-i \cdot, 0)_{g}\left(N_{Y / X}^{\alpha}\right)^{(0)} .
\end{gathered}
$$

By (6.3), (7.10), (7.12) and (7.27), we get (7.18). The proof of our theorem is completed.

TheOREM 7.7. The following identities hold:

$$
T_{K, g}\left(\omega^{M}\right)^{(0)}=0, \quad \log \left(\frac{\left.\right|_{\lambda_{K}^{X}}}{\|_{\lambda^{X}}}\right)^{2}(g)=0 .
$$

Proof. Clearly

$$
\widetilde{\operatorname{ch}}_{g}\left(H^{\bullet}(X, \mathbb{C}), h^{H^{\bullet}(X, \mathbb{C})}, \mathcal{H}_{K}(X, \mathbb{C}), h^{\mathcal{H}} \dot{K}^{\cdot}(X, \mathbb{C})\right)^{(0)}=-\log \left(\frac{\left.\right|_{\lambda_{K}^{X}}}{\|\left.\right|_{\lambda^{X}}}\right)^{2}(g) .
$$

Our theorem now follows from Theorems 6.4, 6.7 and 7.6.

\section{Morse and holomorphic instantons}

In this section, we show that in the case where $K=f^{X}$, the arguments of semi-classical analysis which are needed to study the localization on $Y$ of the Witten instantons [W82] and of the harmonic forms in the holomorphic double complex are not only parallel, but conjugate to each other. In other words, we are dealing with the same objects, which are distorted by a Lefschetz conjugation, which makes them apparently very different.

This section is organized as follows. In $\S 8.1$, we compare the Morse and holomorphic instantons. In $\S 8.2$, we consider the case of a complex vector space, equipped with a linear holomorphic Killing vector field, and with the associated quadratic Morse function. In this case, we relate the associated Witten and Dolbeault-Koszul complexes. In particular we show that the corresponding harmonic forms are related to each other by a Lefschetz transformation. Finally, in $\S 8.3$, we consider the case of a vector bundle, with fibres taken as before. We show that the genus $R(\theta, x)$ and a related genus $J(\theta, x)$ constructed in [BGo01] can in fact be obtained by closely related constructions.

\subsection{Morse and Koszul localization}

We make the same assumptions as in $\S \S 5.7,5.8$ and 5.9. As explained in Remark 5.13, our assumptions are verified in particular if $X$ is connected and $Y$ is non-empty.

By Theorem 5.1, we know that we have a canonical isomorphism,

$$
\mathcal{H}_{K}^{\cdot}(X, \mathbb{C}) \simeq H^{\bullet}(Y, \mathbb{C})
$$

the identification being obtained via the restriction map $j^{*}$. Also by (5.11), we know that the spectral sequence associated to the filtration $G^{\bullet}$ degenerates at $E_{1}$, and moreover,

$$
E_{1} \simeq H^{\bullet}(X, \mathbb{C}) .
$$

Also, by a result of Bismut and Lebeau [BL91, Theorem 9.25], we know that for $T>0$, the dimension of $\operatorname{ker}\left(\bar{\partial}_{T K}^{X}+\bar{\partial}_{T K}^{X *}\right)$ does not depend on $T$, and moreover that for $T \geqslant 1$, there is $c>0$ such that the non-zero eigenvalues of $\bar{\partial}_{T K}^{X}+\bar{\partial}_{T K}^{X *}$ remain in the complement of the interval $[-c, c]$. This fact is essentially related to Theorem 5.1 . 


\section{HOLOMORPHIC AND DE RHAM TORSION}

Now, we consider $H_{\gamma_{K}}^{\cdot}(X, \mathbb{R})$. By $(5.67)$,

$$
H_{\gamma_{K}}^{\cdot}(X, \mathbb{R}) \simeq H^{\bullet}(X, \mathbb{R})
$$

Assume first that $Y$ consists of isolated points. Then $\mu_{K}$ is a Morse function, whose indices at the critical points in $Y$ are even. In this case, the corresponding Morse-Smale complex [T49, Sm61, Sm67] associated to $-\nabla \mu_{K}$ is trivial. More precisely, as explained in [BZ92, ch. 1c], there is a canonical isomorphism

$$
H^{\bullet}(X, \mathbb{R}) \simeq C^{\bullet}(X, \mathbb{R})
$$

In $(8.4), C^{\bullet}(X, \mathbb{R})$ is the $\mathbb{Z}$-graded vector space which is a direct sum of copies of $\mathbb{R}$ indexed by $y \in Y$, the grading being given by the index of $\mu_{K}$.

More generally, since the Bott index of $\mu_{K}$ on the connected components $y$ of the critical manifold $Y$ are even, the same sort of argument shows that we have a canonical isomorphism

$$
H^{\bullet}(X, \mathbb{R}) \simeq \bigoplus_{y \in Y} H^{\cdot+\operatorname{ind}(y)} H^{\bullet}(y, \mathbb{R}) .
$$

Note that the identification in (8.5) involves the de Rham isomorphism of Laudenbach [BZ92], i.e. integration on unstable cells of $-\nabla \mu_{K}$. It should also be pointed out that (8.5) is valid over $\mathbb{Z}$.

Consider now the Witten Laplacian $\left(d_{T \gamma_{K}}+d_{T \gamma_{K}}^{*}\right)^{2}$. For general Morse functions, as explained in [W82], [HSj85], [BZ92, ch. VIII] and [BZ94, § 6], there are in principle non-zero eigenvalues which behave like $e^{-c T}$ with $c>0$ as $T \rightarrow+\infty$, related to the eigenvalues of the combinatorial Laplacian of the associated Morse-Smale complex. If $Y$ only consists of a finite number of points, since the indexes of the critical points of the Morse function $\mu_{K}$ are even, the corresponding Morse-Smale complex is trivial, so that indeed there are no non-zero exponentially small eigenvalues as $T \rightarrow+\infty$. In the general case, $\mu_{K}$ is just Morse-Bott, but the same analysis carries through.

Equations (8.1), (8.2) and (8.3), (8.5) are not directly related, since the canonical isomorphisms which are involved are very different. However, (5.61) shows that the spectral theories for $\bar{\partial}_{T K}^{X}+\bar{\partial}_{T K}^{X *}$ and for $\frac{1}{2} \sqrt{2}\left(d_{\gamma_{T K}}^{X}+d_{\gamma_{T K}}^{X *}\right)$ are the same, and that their harmonic forms correspond by an explicit conjugation. A consequence is that, for example, any argument on small eigenvalues for the first operator is necessarily valid for the other one.

Equation (5.61) has other striking consequences. It indicates in particular not only that the two kinds of analysis involved in studying the holomorphic or de Rham torsions as in [BL91] and in [BZ92, BZ94] are closely related, but also that the two problems are indeed conjugate to each other.

\subsection{A complex Hermitian vector space}

Let $V$ be a finite dimensional vector space, of even dimension $n=2 \ell$ equipped with a scalar product. Let $J^{V}$ be an almost complex structure on $V$ which is antisymmetric. Set $W=\operatorname{ker}\left(J^{V}-i\right) \subset V \otimes_{\mathbb{R}} \mathbb{C}$, so that

$$
V \otimes_{\mathbb{R}} \mathbb{C}=W \oplus \bar{W} .
$$

Then $W$ is a Hermitian vector space. In the sequel, if $Z \in V$, we write $Z=z+\bar{z}$, with $z \in W$, and $\bar{z} \in \bar{W}$ the conjugate of $z$. Let $\omega^{V}$ be the Kähler form of $V$, so that

$$
\omega^{V}=\left\langle\cdot, J^{V} \cdot\right\rangle .
$$

Let $A \in \operatorname{End}(V)$ be antisymmetric and invertible. Assume that $A$ commutes with $J^{V}$. Then $A$ also acts on $W$ as a skew-adjoint invertible element of $\operatorname{End}(W)$.

Set

$$
K=A z
$$




\section{J.-M. Bismut}

Then $K$ is a holomorphic section of $W$ which vanishes at 0 . The corresponding real vector field $K_{\mathbb{R}}$ is given by

$$
K_{\mathbb{R}}=A Z \text {. }
$$

Now we will use the notation of $\S 5$ with $V=X$. In particular, as in (5.53), set

$$
\gamma_{K}=-i_{K_{\mathbb{R}}} \omega^{V}
$$

By (5.61), we get

$$
e^{-\pi M / 4} i^{N^{(1,0)}} \frac{\sqrt{2}}{2}\left(d_{\gamma_{K}}^{V}+d_{\gamma_{K}}^{V, *}\right) i^{-N^{(1,0)}} e^{\pi M / 4}=\bar{\partial}_{K}^{V}+\bar{\partial}_{K}^{V *} .
$$

Let $e_{1}, \ldots, e_{n}$ be an orthonormal basis of $V$.

Proposition 8.1. The following identities hold:

$$
\begin{gathered}
\frac{1}{2}\left(d_{\gamma_{K}}^{V}+d_{\gamma_{K}}^{V, *}\right)^{2}=-\frac{1}{2} \Delta^{V}+\frac{1}{2}|A Z|^{2}+\frac{1}{2} c\left(e_{i}\right) \widehat{c}\left(J^{V} A e_{i}\right), \\
\left(\bar{\partial}_{K}^{V}+\bar{\partial}_{K}^{V *}\right)^{2}=-\frac{1}{2} \Delta^{V}+\frac{1}{2}|A Z|^{2}+\frac{i}{2} c_{\bar{W}}\left(e_{i}\right) c_{W}\left(J^{V} A e_{i}\right) .
\end{gathered}
$$

Proof. This follows from a trivial computation which is left to the reader.

Clearly both operators in (8.12) are harmonic oscillators on $V$, and so they have a discrete spectrum.

Set

$$
B=\frac{A}{|A|}
$$

Then $B$ is antisymmetric and invertible, and commutes with $J^{V}$, so that it preserves $W$ and $\bar{W}$. If $X, Y \in V$, set

$$
\theta_{B}(X, Y)=\langle X, B Y\rangle
$$

Then $\theta_{B}$ is a symplectic form on $V$, which is of type $(1,1)$.

Put

$$
\mu_{K}(Z)=\frac{1}{2}\left\langle J^{V} A Z, Z\right\rangle
$$

Then $\mu_{K}$ is a Morse function, and

$$
d \mu_{K}=\gamma_{K}
$$

Clearly $J^{V} A \in \operatorname{End}(V)$ is symmetric. Let $V=V_{+} \oplus V_{-}$be the orthogonal splitting of $V$ corresponding to the positive and negative eigenvalues of $J^{V} A$. Then $V_{+}$and $V_{-}$are stable by $J^{V}$. The form $\omega^{V}$ restricts to a symplectic $(1,1)$ form $\omega^{V_{-}}$on $V_{-}$. Let $\kappa^{V_{-}}$be the corresponding volume form on $V_{-}$.

TheOREM 8.2. The kernel of $\left(\bar{\partial}_{K}^{V}+\bar{\partial}_{K}^{V *}\right)^{2}$ is one-dimensional, and is spanned by

$$
\beta=\exp \left(\theta_{B}-\frac{1}{2}\langle|A| Z, Z\rangle\right) \text {. }
$$

Moreover,

$$
\begin{aligned}
& \bar{\partial}_{K}^{V} \beta=0, \quad \partial_{K} \beta=0, \\
& \bar{\partial}_{K}^{V *} \beta=0, \quad \partial_{K}^{*} \beta=0 .
\end{aligned}
$$

The kernel of $\frac{1}{2}\left(d_{\gamma_{K}}^{V}+d_{\gamma_{K}}^{V, *}\right)^{2}$ is one-dimensional and spanned by

$$
\delta=\exp \left(-\frac{1}{2}\langle|A| Z, Z\rangle\right) \kappa^{V_{-}} .
$$




\section{HOLOMORPHIC AND DE RHAM TORSION}

Moreover,

$$
d_{\gamma_{K}}^{V} \delta=0, \quad d_{\gamma_{K}}^{V *} \delta=0
$$

Finally,

$$
i^{-N^{(1,0)}} e^{\pi M / 4} \beta=(-i)^{\operatorname{dim} V_{-} / 2} 2^{\operatorname{dim} V / 2} \delta .
$$

Proof. The fact that the kernel of $\left(\bar{\partial}_{K}^{V}+\bar{\partial}_{K}^{V *}\right)^{2}$ is one-dimensional was proved in [B90a, Theorem 1.6] when $A=J^{V}$. A trivial rescaling argument shows that this is still the case in general. The same reference, or a trivial computation, shows that (8.18) holds.

The second part of our theorem is established in [W82]. Equation (8.20) can be given a direct easy proof.

Now to establish (8.21), it is enough to consider the case where $n=2$. In this case $J^{V} A$ is just multiplication by $\lambda \in \mathbb{R}^{*}$. Then

$$
\theta^{B}=-\operatorname{sgn}(\lambda) \omega^{V}
$$

Using (1.5) and (1.6), we get

$$
M 1=\omega^{V}, \quad M \omega^{V}=-1 .
$$

By (8.23), we find that

$$
e^{\pi M / 4} 1=\frac{\sqrt{2}}{2}\left(\omega^{V}+1\right), \quad e^{\pi M / 4} \omega^{V}=\frac{\sqrt{2}}{2}\left(\omega^{V}-1\right) .
$$

By (8.22) and (8.23), we get

$$
e^{\pi M / 4} e^{\theta_{B}}=e^{\pi M / 4}\left(1-\operatorname{sgn}(\lambda) \omega^{V}\right),
$$

so that

$$
\begin{aligned}
e^{\pi M / 4} e^{\theta_{B}} & =\sqrt{2} \quad \text { if } \lambda>0, \\
& =\sqrt{2} \omega^{V} \quad \text { if } \lambda<0 .
\end{aligned}
$$

By (8.17), (8.19) and (8.26), we get (8.21). The proof of our theorem is completed.

Remark 8.3. Equation (8.21) is natural in view of (8.11). Also note that the results of the present section can be viewed as special cases of the arguments given in $\S 8.1$. Theorem 8.2 is interesting. Indeed in the proof of the embedding formulas of [BL91, B97], a key idea is to reduce the embedding of $Y$ into $X$ to the embedding of $Y$ in the normal bundle $N_{Y / X}$. The form $\beta$ plays a critical role to relate the holomorphic Hodge theory of $X$ to the Hodge theory of $Y$. For the Witten complex [W82] associated to a Morse-Bott function $f$, the form $\delta$ plays a similar role, to relate the de Rham Hodge theory of $X$ to the corresponding theory on $Y$. As demonstrated by (8.21), the two sorts of localizations are indeed identical.

\subsection{The real and holomorphic superconnections of a vector bundle}

Let now $S$ be a complex manifold. Let $W$ be a holomorphic Hermitian vector bundle on $S$, let $V$ be the underlying real vector bundle, and let $J^{V}$ be the associated complex structure. Set $n=\operatorname{dim} V$. Let $\nabla^{W}$ be the holomorphic Hermitian connection on $W$, and let $R^{W}$ be its curvature. In the sequel, we will take $K=i z$.

For $T \in \mathbb{R}_{+}^{*}$, we define $B_{T K, t}$ in $\S 5.10$. Then

$$
B_{T K, t}=\sqrt{t}\left(\bar{\partial}_{T K}^{V}+\bar{\partial}_{T K}^{V, *}\right)+\nabla^{\Omega^{\bullet}(V, \mathbb{C})}-\frac{1}{2 \sqrt{2 t}} c_{\bar{W}}\left(R^{W} Z\right) .
$$


We still define the fibrewise 1-form $\gamma_{T K}$ as in (8.10) and the function $\mu_{T K}$ on the total space of $V$ as in (8.15). We define $C_{\gamma_{T K}}=C_{\gamma_{T K}, 1}$ as in $\S 5.11$. We get

$$
C_{\gamma_{T K}, t}=\frac{\sqrt{t}}{2}\left(d_{\gamma_{T K}}^{V}+d_{\gamma_{T K}}^{V, *}\right)+\nabla^{\Omega^{\bullet}(V, \mathbb{R})}-\frac{1}{2 \sqrt{t}} c\left(R^{W} Z\right) .
$$

By (5.85) in Theorem 5.14, we get

$$
e^{-\pi M / 4} i^{N^{(1,0)}} C_{\gamma_{T K}, 2 t} i^{-N^{(1,0)}} e^{\pi M / 4}=B_{T K, t} .
$$

Proposition 8.4. The following identities hold:

$$
\begin{gathered}
\operatorname{Tr}_{\mathrm{s}}\left[g \exp \left(-B_{T K, 1}^{2}\right)\right]=\operatorname{Tr}_{\mathrm{s}}\left[g \exp \left(-C_{T K, 2}^{2}\right)\right]=1 \\
\operatorname{Tr}_{\mathrm{s}}\left[g N^{(1,0)} \exp \left(-B_{T K, 1}^{2}\right)\right]=\operatorname{Tr}_{\mathrm{s}}\left[g\left(-\frac{1}{2} \mathcal{N}+i \frac{M}{2}\right) \exp \left(-C_{T K, 2}^{2}\right)\right]+\frac{n}{4} .
\end{gathered}
$$

The form $\operatorname{Tr}_{\mathrm{s}}\left[g N^{(1,0)} \exp \left(-B_{T K, 1}^{2}\right)\right]-n / 4$ is purely imaginary.

Proof. By (8.29), we get the first equality in (8.30). By proceeding as in the proofs of Propositions 4.7 and 6.11, we get the second equality in the first row of (8.30). Using Theorem 1.5 and (8.29), we obtain

$$
\operatorname{Tr}_{\mathrm{s}}\left[g N^{(1,0)} \exp \left(-B_{T K, 1}^{2}\right)\right]=\operatorname{Tr}_{\mathrm{s}}\left[\left(-\frac{1}{2} \mathcal{N}+\frac{n}{4}+\frac{i}{2} M\right) \exp \left(-C_{T K, 2}^{2}\right)\right]
$$

Using (8.31), we get the second identity in (8.30). Using the fact that the operators $N^{(1,0)}$ and $N^{(0,1)}$ are conjugate, and that the other operators which appear on the right-hand side of the second identity in (8.30) are real, we obtain the last part of our proposition.

In [B94, Definition 6.1], the genus $R_{g}(E)$ is essentially obtained as the derivative at $s=0$ of the Mellin transform in the variable $T \in \mathbb{R}_{+}^{*}$ of the form $\Phi \operatorname{Tr}_{\mathrm{s}}\left[g N^{(1,0)} \exp \left(-B_{T K, 1}^{2}\right)\right]$. By Proposition 8.4, we find that the class $\Phi^{-1} R_{g}(E)$ should be purely imaginary, as confirmed by (6.3).

Now we recall the definition in [BGo01, ch. 4] of a formal power series $J(\theta, x)$ formally closely related to $R(\theta, x)$.

Definition 8.5. For $\theta \in \mathbb{R} / 2 \pi \mathbb{Z}$, let $J(\theta, x)$ be the formal power series,

$$
J(\theta, x)=\frac{1}{4}\left[\sum_{\substack{n \geqslant 0 \\ n \text { even }}}\left\{\sum_{j=1}^{n} \frac{1}{j} \zeta(\theta,-n)+2 \frac{\partial \zeta}{\partial s}(\theta,-n)\right\} \frac{x^{n}}{n !}+\sum_{\substack{n \geqslant 0 \\ n \text { odd }}} i\left\{\sum_{j=1}^{n} \frac{1}{j} \eta(\theta,-n)+2 \frac{\partial \eta}{\partial s}(\theta,-n)\right\} \frac{x^{n}}{n !}\right] .
$$

It should be pointed out that $\eta(\theta,-n)$ vanishes for $n \in \mathbb{N}^{*}$ odd, and $\zeta(\theta,-n)=0$ for $n \in \mathbb{N}^{*}$ even. So the terms containing $\sum_{j=1}^{n} 1 / j$ in (8.32) can be eliminated.

Let $J_{g}(E)$ be the corresponding genus which is constructed as in (6.5). As explained in [BGo01, ch. 4], the genus $J_{g}(E)$ is essentially obtained as the derivative at $s=0$ of the Mellin transform in the variable $T \in \mathbb{R}_{+}^{*}$ of the form $-\operatorname{Tr}_{\mathrm{s}}\left[g(N / 2) \exp \left(-C_{T K, 1}^{2}\right)\right]$. Note that the precise procedure is more complicated than in [B94], but it is still natural from the point of secondary characteristic classes.

Now we use (8.30). We obtain the remarkable fact that the genera $R_{g}(E)$ and $J_{g}(E)$ can both be expressed in terms of Mellin transforms of the forms $\operatorname{Tr}_{\mathrm{s}}\left[g(M / 2) \exp \left(-C_{T K, 1}^{2}\right)\right], \operatorname{Tr}_{\mathrm{S}}[g \mathcal{N}$ $\left.\exp \left(-C_{T K, 1}^{2}\right)\right]$ and $\operatorname{Tr}_{\mathrm{s}}\left[g(N / 2) \exp \left(-C_{T K, 1}^{2}\right)\right]$. This suggests that the underlying $s l_{2}$ symmetry in (1.6) should play a role in explaining the formal similarities in the series (6.3) and (8.32) which define $R(\theta, x)$ and $J(\theta, x)$. 


\section{HolOMORPHIC AND DE RHAM TORSION}

\section{ACKNowledGements}

The author is indebted to two referees for their very careful reading of the paper, and their helpful remarks.

\section{REFERENCES}

ABo67 M. F. Atiyah and R. Bott, A Lefschetz fixed point formula for elliptic complexes. I, Ann. of Math. (2) 86 (1967), 374-407.

ABo68 M. F. Atiyah and R. Bott, A Lefschetz fixed point formula for elliptic complexes. II. Applications, Ann. of Math. (2) $8 \mathbf{8}$ (1968), 451-491.

AS71 M. F. Atiyah and I. M. Singer, The index of elliptic operators. IV, Ann. of Math. (2) 93 (1971), $119-138$.

BeGeV92 N. Berline, E. Getzler, and M. Vergne, Heat kernels and Dirac operators, Grundl. Math. Wiss., Band 298 (Springer, Berlin, 1992).

BeV83 N. Berline and M. Vergne, Zéros d'un champ de vecteurs et classes caractéristiques équivariantes, Duke Math. J. 50 (1983), 539-549.

B86 J.-M. Bismut, The Atiyah-Singer index theorem for families of Dirac operators: two heat equation proofs, Invent. Math. 83 (1986), 91-151.

B90a J.-M. Bismut, Koszul complexes, harmonic oscillators, and the Todd class, J. Amer. Math. Soc. 3 (1990), 159-256 (with an appendix by the author and C. Soulé).

B90b J.-M. Bismut, Superconnection currents and complex immersions, Invent. Math. 99 (1990), 59113.

B92 J.-M. Bismut, Bott-Chern currents, excess normal bundles and the Chern character, Geom. Funct. Anal. 2 (1992), 285-340.

B94 J.-M. Bismut, Equivariant short exact sequences of vector bundles and their analytic torsion forms, Compositio Math. 93 (1994), 291-354.

B95 J.-M. Bismut, Equivariant immersions and Quillen metrics, J. Differential Geom. 41 (1995), $53-157$.

B97 J.-M. Bismut, Holomorphic families of immersions and higher analytic torsion forms, Astérisque 244 (1997).

BF86 J.-M. Bismut and D. S. Freed, The analysis of elliptic families. I. Metrics and connections on determinant bundles, Comm. Math. Phys. 106 (1986), 159-176.

BGS88a J.-M. Bismut, H. Gillet and C. Soulé, Analytic torsion and holomorphic determinant bundles. I. Bott-Chern forms and analytic torsion, Comm. Math. Phys. 115 (1988), 49-78.

BGS88b J.-M. Bismut, H. Gillet and C. Soulé, Analytic torsion and holomorphic determinant bundles. II. Direct images and Bott-Chern forms, Comm. Math. Phys. 115 (1988), 79-126.

BGS88c J.-M. Bismut, H. Gillet and C. Soulé, Analytic torsion and holomorphic determinant bundles. III. Quillen metrics on holomorphic determinants, Comm. Math. Phys. 115 (1988), 301-351.

BGS90 J.-M. Bismut, H. Gillet, and C. Soulé, Complex immersions and Arakelov geometry, in The Grothendieck Festschrift, vol. I (Birkhäuser, Boston, MA, 1990), 249-331.

BGo01 J.-M. Bismut and S. Goette, Families torsion and Morse functions, Astérisque 275 (2001).

BK92 J.-M. Bismut and K. Köhler, Higher analytic torsion forms for direct images and anomaly formulas, J. Algebraic Geom. 1 (1992), 647-684.

BL91 J.-M. Bismut and G. Lebeau, Complex immersions and Quillen metrics, Publ. Math. Inst. Hautes Études Sci. 74 (1991).

BLo95 J.-M. Bismut and J. Lott, Flat vector bundles, direct images and higher real analytic torsion, J. Amer. Math. Soc. 8 (1995), 291-363.

BM02 J.-M. Bismut and X. Ma, Holomorphic immersions and equivariant torsion forms, Preprint, Université Paris-Sud, Orsay (2002). 


\section{Holomorphic AND DE RHAM TORSION}

BZ92 J.-M. Bismut and W. Zhang, An extension of a theorem by Cheeger and Müller, Astérisque 205 (1992), 235 (with an appendix by François Laudenbach).

BZ94 J.-M. Bismut and W. Zhang, Milnor and Ray-Singer metrics on the equivariant determinant of a flat vector bundle, Geom. Funct. Anal. 4 (1994), 136-212.

Bo67 R. Bott, Vector fields and characteristic numbers, Michigan Math. J. 14 (1967), 231-244.

CE56 H. Cartan and S. Eilenberg, Homological algebra (Princeton University Press, Princeton, NJ, 1956).

CaL73 J. B. Carrell and D. I. Lieberman, Holomorphic vector fields and Kähler manifolds, Invent. Math. 21 (1973), 303-309.

D68 P. Deligne, Théorème de Lefschetz et critères de dégénérescence de suites spectrales, Publ. Math. Inst. Hautes Études Sci. 35 (1968), 259-278.

DuH82 J. J. Duistermaat and G. J. Heckman, On the variation in the cohomology of the symplectic form of the reduced phase space, Invent. Math. 69 (1982), 259-268.

DuH83 J. J. Duistermaat and G. J. Heckman, Addendum to: 'On the variation in the cohomology of the symplectic form of the reduced phase space', Invent. Math. 72 (1983), 153-158.

GS91 H. Gillet and C. Soulé, Analytic torsion and the arithmetic Todd genus, Topology 30 (1991), 21-54 (with an appendix by D. Zagier).

GrHa78 P. Griffiths and J. Harris, Principles of algebraic geometry, Pure and Applied Mathematics (WileyInterscience, New York, 1978).

GrT84 P. Griffiths and L. Tu, Variation of Hodge structure, Topics in transcendental algebraic geometry (Princeton, NJ, 1981/1982) (Princeton University Press, Princeton, NJ, 1984), 3-28.

HSj85 B. Helffer and J. Sjöstrand, Puits multiples en mécanique semi-classique. IV. Étude du complexe de Witten, Comm. Partial Differential Equations 10 (1985), 245-340.

M00 X. Ma, Submersions and equivariant Quillen metrics, Ann. Inst. Fourier (Grenoble) 50 (2000), $1539-1588$.

Q85 D. Quillen, Superconnections and the Chern character, Topology 24 (1985), 89-95.

RS71 D. B. Ray and I. M. Singer, R-torsion and the Laplacian on Riemannian manifolds, Adv. Math. 7 (1971), 145-210.

RS73 D. B. Ray and I. M. Singer, Analytic torsion for complex manifolds, Ann. of Math. (2) 98 (1973), 154-177.

Sm61 S. Smale, On gradient dynamical systems, Ann. of Math. (2) 74 (1961), 199-206.

Sm67 S. Smale, Differentiable dynamical systems, Bull. Amer. Math. Soc. 73 (1967), 747-817.

T49 R. Thom, Sur une partition en cellules associée à une fonction sur une variété, C. R. Acad. Sci. Paris 228 (1949), 973-975.

W82 E. Witten, Supersymmetry and Morse theory, J. Differential Geom. 17 (1982), 661-692.

Z90 W. P. Zhang, A remark on a residue formula of Bott, Acta Math. Sinica (N.S.) 6 (1990), 306-314 [A Chinese summary appears in Acta Math. Sinica 34 (1991), 718.]

Jean-Michel Bismut Jean-Michel.Bismut@math.u-psud.fr

Département de Mathématique, Université Paris-Sud, Bâtiment 425, 91405 Orsay, France 